\title{
A GLOBAL THEORY OF INTERNAL SOLITARY WAVES IN TWO-FLUID SYSTEMS
}

\author{
C. J. AMICK AND R. E. L. TURNER
}

\begin{abstract}
The problem analyzed is that of two-dimensional wave motion in a heterogeneous, inviscid fluid confined between two rigid horizontal planes and subject to gravity $g$. It is assumed that a fluid of constant density $\rho_{+}$ lies above a fluid of constant density $\rho_{-}>\rho_{+}>0$ and that the system is nondiffusive. Progressing solitary waves, viewed in a moving coordinate system, can be described by a pair $(\lambda, w)$, where the constant $\lambda=g / c^{2}, c$ being the wave speed, and where $w(x, \eta)+\eta$ is the height at a horizontal position $x$ of the streamline which has height $\eta$ at $x= \pm \infty$. It is shown that among the nontrivial solutions of a quasilinear elliptic eigenvalue problem for $(\lambda, w)$ is an unbounded connected set in $\mathbf{R} \times\left(H_{0}^{1} \cap C^{0,1}\right)$. Various properties of the solution are shown, and the behavior of large amplitude solutions is analyzed, leading to the alternative that internal surges must occur or streamlines with vertical tangents must occur.
\end{abstract}

1. Introduction. The study of single-crested progressing gravity waves was initiated over a century ago. It began with the observations by Russell $[\mathbf{2 4}]$ of what he termed solitary waves, which progressed without change of form over a considerable distance on the Glasgow-Edinburgh Canal. The mathematical analysis of this wave motion on the surface of water, begun in the nineteenth century, has undergone a rapid development in the last three decades, due to the scattering theory for the Korteweg-de Vries equation, which models the motion of long waves, and due to the development of techniques in nonlinear analysis allowing for the study of finite amplitude motions (cf. $[\mathbf{5}, \mathbf{1 9}]$ and references given there). The work on surface waves has many parallels in the study of waves in fluids with variable density. In the case of a heterogeneous fluid with a free upper surface, gravity waves still occur, in analogy with surface waves in a fluid of constant density (cf. $[\mathbf{2 3}, \mathbf{2 5}$, 27]). What is distinctive about a fluid with density stratification, however, is the presence of waves which are predominantly due to the stratification and not to the free surface. These waves, called internal waves, exist in a heterogeneous fluid even when it is confined between horizontal boundaries, a configuration which precludes gravity waves in a fluid of constant density.

For surveys of earlier work on permanent waves in stratified fluids and for more complete references than given here, we refer the reader to the articles by Benjamin [8], Bona, Bose, and Turner [10], and Turner [26].

Received by the editors January 26, 1986.

1980 Mathematics Subject Classification (1985 Revision). Primary 35J60, 76C10.

Key words and phrases. Internal wave, solitary wave, internal bore, bifurcation.

Sponsored by the United States Army under Contract No. DAAG29-80-C-0041 and the National Science Foundation under Grant Nos. DMS-8203338 and MCS-8200406. 
Our concern in this paper is with progressing solitary waves in a system consisting of two fluids of differing densities confined in a channel of unit depth and infinite horizontal extent. The undisturbed state consists of a fluid of constant density $\rho_{-}$ having depth $h$ in contact with the lower, planar boundary and a second fluid of constant positive density $\rho_{+}<\rho_{-}$, lying above the first and having depth $1-h$, so as to fill the channel. The motion studied is assumed to be two-dimensional, inviscid, incompressible, and nondiffusive. We shall always remove the time variable from consideration by taking a moving coordinate system in which the flow is steady and symmetric about the vertical axis.

The problem of finding nontrivial wave motion can be posed in terms of a pair of harmonic stream functions, $\psi^{+}$for the upper fluid and $\psi^{-}$for the lower fluid. At the interface between the two fluids, a free boundary in this setting, one must impose the continuity of pressure and of normal velocity components. A direct analysis of the problem in this setting might be attempted along the lines of the work on two fluid jets by Alt, Caffarelli, and Friedman $[\mathbf{1}, \mathbf{2}]$. However, they make essential use of having a minimizer for an appropriate functional and must do a delicate analysis to show that the interface separating the two fluids is of class $C^{1}$. In the present context the analogous minima would correspond to trivial parallel flows (cf. [10, 26]) and the nontrivial flows, to what are undoubtedly saddle points. By taking a different approach we obtain a global picture of the solitary wave solutions and show that for each wave the interface between the two fluids is analytic.

The approach taken in this paper is to approximate the discontinuous density function by a sequence of smooth density profiles and to obtain sufficiently good estimates in the smooth case to allow appropriate limits to be taken. In this way we also show that discontinuous densities and smooth, rapidly varying densities give rise to motions which are close to each other. Using methods of global bifurcation, the first author in [4] gave a global theory for solitary waves in the case of an arbitrary smooth density. The solutions are given by stream functions which are smooth everywhere in the channel. This approach is not limited by the particular shape of the density profile, as was the variatonal approach used in [10]. However, a limiting process to obtain solutions for the density having a jump, from $\rho_{-}$to $\rho_{+}$, would still entail a confrontation with an unknown free boundary.

An alternative is to use semi-Lagrangian coordinates. To describe these coordinates we first note that the stream function referred to in the last paragraph differs from the usual one in having an intervening density factor (see formula 2.14). Using accepted terminology we call it a pseudo-stream-function and denote it by $\psi=\psi(x, y)$, where $x$ is a horizontal coordinate and $y$ is a vertical coordinate chosen so that gravity acts in the negative $y$ direction. In the semi-Lagrangian formulation one uses $x$ and $\psi$ as independent variables and $y$ as a dependent variable. In this formulation the equation to be considered is highly singular. However, what would be free boundaries in the spatial domain correspond to coordinate lines, $\psi=$ constant, in the new variables. This formulation was used by Ter-Krikorov [25] in combination with fixed point methods to show the existence of small amplitude periodic and solitary waves in the case of a fixed or free boundary at the upper surface of a fluid with smoothly varying density. Using this formulation, but applying a variational method, the second author in [26 and 27] studied the two-fluid system under consideration here as well as a multiple-fluid system with a free upper sur- 
face. The existence of periodic and solitary wave was shown, the latter waves being obtained as the limit of periodic ones with ever-increasing periods. In the present paper, to obtain large amplitude waves in the case of a discontinuous density, we work principally with the semi-Lagrangian formulation, but as a starting point use the solutions provided by [4]. It will be shown that there is a global theory for a discontinuous density analogous to that derived in [4] for a smooth density.

In $\S 2.1$ the passage from the postulated physical model to the relevant equations is made. The equations are of two types. The first is a semilinear elliptic equation for a pseudo-stream-function $\psi(x, y)$. The second is a singular, quasilinear elliptic equation which governs the deviation of streamlines from parallel flow when semi-Lagrangian coordinates are used. In both equations there enters an unknown parameter which describes the speed $c$ of a progressive wave as seen in "laboratory" coordinates. In the second formulation it appears explicitly as an eigenvalue parameter $\lambda=g / c^{2}$, where $g$ is the gravity constant.

In $\S 2.2$ the main results of the paper are stated in Theorem 2.1. It describes a branch $S$ of solution pairs $(\lambda, w)$, where $w=w\left(x_{1}, x_{2}\right)$ is the deviation at the horizontal position $x_{1}$ of the streamline which has height $x_{2}$ at $x_{1}= \pm \infty$. If $T$ denotes the domain of the pairs $\left(x_{1}, x_{2}\right)$, the branch is unbounded and connected in $\mathbf{R} \times\left(H_{0}^{1}(T) \cap C^{0,1}(\bar{T})\right)$. It emanates from a pair $\left(\lambda_{d}, 0\right)$, where $\lambda_{d}$ is a particular function of $\rho_{+}, \rho_{-}$, and $h$. The speed $c_{d}=\left(g / \lambda_{d}\right)^{1 / 2}$ is a critical speed for the stratified configuration, the analogue of the speed $\sqrt{g h}$ for long waves on the surface of water of depth $h$. For all nontrivial solutions in $S, 0<\lambda<\lambda_{d}$, and thus the associated speeds are supercritical, a result consistent with all analyses of solitary wave phenomena.

The sign of another parameter $e$, a function of $\rho_{+}, \rho_{-}$, and $h$, predicts the sign of streamline displacements. If $e>0$, the undistributed state will allow waves of elevation, while for $e<0$ it allows waves of depression. The phenomenon described here, as distinct from that associated with simple eigenvalue bifurcation, is one-sided. If $e>0$ there are elevation waves but no depression waves near the bifurcation point $\left(\lambda_{d}, 0\right)$. Finally, all waves are symmetric about their crests and decay monotonically and exponentially to zero as $x \rightarrow \pm \infty$.

The core of the proof of Theorem 2.1 is carried out in the course of $\S \S 3$ through 6. We begin with a sequence $\rho_{n}, n=1,2,3, \ldots$, of smooth density profiles in the undisturbed channel configuration, which, as $n \rightarrow \infty$, converge to the given discontinuous density function. It is shown in $\S 3$ that for each $n$ a subset of the pseudo-stream-functions from [4] provides an unbounded, closed, connected set of solutions $(\lambda, w)$ in $\mathbf{R} \times H_{0}^{1}(T)$ with which to work in the new coordinates. In $\S 4 \mathrm{a}$ general scheme for the limiting process is given. Then, after imposing restrictions on the gradient of $w$, regularity estimates for solutions are derived, the estimates being independent of $n$. These estimates are used in $\S 5$ to obtain connected sets of solutions in $\mathbf{R} \times H_{0}^{1}(T)$ for the case of a discontinuous density. In $\S 6$ the solution sets are examined in the stronger topology of $\mathbf{R} \times\left(H_{0}^{1}(T) \cap C^{0,1}(\bar{T})\right)$. Here it is shown that connectedness is preserved. The next step concerns the restrictions imposed on the gradient of $w$ in $\S 4$. It must be shown that for any solution $(\lambda, w)$ under consideration, the gradient of $w$ does not take values in a range that would make the underlying elliptic equation singular. This is accomplished by returning to the original formulation involving the pseudo-stream-functions $\psi^{ \pm}$and obtaining 
bounds on their gradients, that is, bounds for the velocities in the flow domain. These bounds translate into the desired estimates for the gradient of $w$.

In $\S 7$ results constituting the proof of Theorem 2.1 are collected. Many properties of solutions are obtained in the course of proving the existence of the set $S$. To these are added the proofs that the interface is analytic and that depression (elevation) waves are absent near the bifurcation point $\left(\lambda_{d}, 0\right)$ if $e>0(e<0)$.

Numerical studies have been made of periodic, interfacial, gravity waves for two unbounded fluids of differing densities (cf. $[\mathbf{1 7}, \mathbf{2 8}])$. It is found that along a branch of waves of fixed wavelength, the separating streamline steepens, eventually manifesting a vertical tangent and, past that, an overhanging region in which heavier fluid lies above the lighter one. From these studies one might conjecture that, along the branch of solutions $S$ found in this paper, $w$ becomes large in $C^{0,1}$. In fact, the introduction of the $C^{0,1}$ topology, apart from having a crucial role in the estimates of $\S 4$, was suggested by the numerical work. $\S 8$ concerns the behavior of large amplitude solutions on the branch $S$. The fact of having a channel of finite depth and solitary rather than periodic waves may change the character of large amplitude waves. We pursue the implications of assuming that wave profiles do not steepen to the point of having vertical profiles. A first consequence is that there must exist a solution $(\tilde{\lambda}, \tilde{w})$ of the flow equations for which $\tilde{w}$ is not in $L^{2}(T)$. Rather, at $x= \pm \infty$ it asymptotically approaches a nontrivial parallel flow, a "conjugate" flow in the terminology of Benjamin [9]. A further consequence is the existence of an "internal surge" of predictable size and speed. As yet, no contradiction has arisen from this train of arguments and so the large amplitude behavior remains an open question. In a project in progress the second author and J.-M. Vanden-Broeck [29] are carrying out a numerical study of the solitary wave patterns shown to exist here and hope to shed light on their behavior.

\section{Formulation of the flow problem and results.}

2.1. The flow equations. Here we describe the physical model under consideration and the passage to a boundary-value problem for a partial differential equation. The boundary-value problem, in turn, is formulated in essentially two ways, and the interplay between these is used in our analysis. Consider an incompressible, inviscid fluid acted on by gravity and restrict attention to a two-dimensional flow confined to and filling a region $S=\{(x, y) \mid-\infty<x<\infty,-h<y<1-h\}$. The acceleration of gravity has magnitude $g$ in the negative $y$ direction. Further assumed characteristics of the fluid which make propagation of permanent waves possible and their study tractable are that it is heterogeneous and nondiffusive. To explain the last term it is worthwhile to first describe a diffusive system. Consider a mixture of two components, say water and a dissolved salt. In a mixture the molecules can trade places without having any net mean-molar velocity. However, if the molecules have different masses, there can be a net movement of mass. Let $\mathbf{q}$ denote the mean-molar velocity and $\mathbf{Q}$, the mean-mass velocity (cf. [11, Chapter 1]). Let $\rho$ denote the density of the fluid, and suppose, to begin with, that $\rho$ is a smooth function of space and time. Subsequently we shall formulate the problem for a discontinuous density. If $\nu$ denotes the diffusion coefficient (assumed the same for the two types of molecules), then the Fick diffusion law (cf. $\left[\mathbf{7}^{1} ; \mathbf{1 1}\right.$, p. $\left.23 ; 14\right]$ )

\footnotetext{
${ }^{1}$ We wish to thank the authors for a discussion of the diffusive model.
} 
gives

$$
\mathbf{Q}=\mathbf{q}-\nu \nabla \rho / \rho
$$

The Euler momentum equation is

$$
\rho(\partial \mathbf{Q} / \partial t+(\mathbf{Q} \cdot \nabla) \mathbf{Q})=-\nabla p-\rho g \mathbf{k}
$$

where $p$ is the pressure and $\mathbf{k}$ is a unit vector in the $y$ direction. Conservation of mass takes the form

$$
\partial \rho / \partial t+\operatorname{div}(\rho \mathbf{Q})=0
$$

and the volume incompressibility, the form

$$
\operatorname{div} \mathbf{q}=0 .
$$

A set of equations for $\rho$ and $\mathbf{q}$ is obtained by using (2.1) in (2.2) and (2.3). We will seek a wave motion which has a permanent form when viewed in a coordinate system moving in the $x$ direction with constant speed and in such a coordinate system the equations will have no explicit time dependence. To avoid introducing new notation, let us assume for the remainder of the paper that all quantities are referred to the moving system, but retain the notation already introduced. With no time derivatives present, the equations for $\rho$ and $\mathbf{q}$ reduce to

$$
\begin{gathered}
\rho(\mathbf{q} \cdot \nabla) \mathbf{q}+O(\nu)=-\nabla p-\rho g \mathbf{k}, \\
\mathbf{q} \cdot \nabla \rho-\nu \Delta p=0, \\
\operatorname{div} \mathbf{q}=0,
\end{gathered}
$$

where $O(\nu)$ refers to terms of order $\nu$ for $\nu$ small. Time scales for diffusion (e.g., in stratified salt solutions) are on the order of hours, while a wave of the type sought here will pass in a matter of seconds. Thus, diffusion plays an insignificant role in the wave propagation phenomenon. By a nondiffusive fluid we merely mean a fluid governed by (2.5) $-(2.7)$ with $\nu=0$. The resulting equations are the standard ones used for nondiffusive stratified fluids, but the condition $\mathbf{q} \cdot \nabla \rho=0$, attributed to nondiffusivity, is hereby rationalized. Otherwise, it would seem to arise from using a single solenoidal field $\mathbf{q}$ together with mass conservation and not have any clear connection with diffusion (or lack of it).

The equations which result from setting $\nu=0$ in (2.5)-(2.7) and which are the basis for our analysis are:

$$
\begin{gathered}
\rho(\mathbf{q} \cdot \nabla) \mathbf{q}=-\nabla p-\rho g \mathbf{k}, \\
\mathbf{q} \cdot \nabla \rho=0, \\
\operatorname{div} \mathbf{q}=0 .
\end{gathered}
$$

For use in the sequel we let $\mathbf{q}=(U, V)$, where $U$ and $V$ denote the horizontal and vertical components of velocity, respectively. It will be assumed that in the original "laboratory" coordinates the fluid is at rest at $x= \pm \infty$ and there has a positive density $\rho_{\infty}(y)$ which is nonincreasing in $y$ and satisfies $\rho_{\infty}(-h)>\rho_{\infty}(1-h)$. Thus if a wave under consideration is moving from right to left in the "laboratory" with 
speed $c>0$, a corresponding boundary-value problem in the new coordinates is to find $\mathbf{q}(x, y), p(x, y), \rho(x, y)$, and $c$ satisfying $(2.8)-(2.10)$ and

$$
\begin{gathered}
\lim _{|x| \rightarrow \infty} \rho(x, y)=\rho_{\infty}(y), \\
\lim _{|x| \rightarrow \infty}(U, V)=(c, 0), \\
V=0 \text { on } \partial S,
\end{gathered}
$$

where $\partial S$ denotes the boundary of $S$. Note that $\mathbf{q}(x, y)=(c, 0), p(x, y)=$ $-\int_{0}^{y} \rho_{\infty}(s) g d s$, and $\rho(x, y)=\rho_{\infty}(y)$ is a solution of $(2.8)-(2.13)$ for any real $c$ and will be referred to as a trivial solution. We shall find nontrivial solutions of the boundary-value problem (2.8)-(2.13), properly interpreted, for a piecewise constant density $\rho_{d}(y)$, given as $\rho_{\infty}(y)$ and shall do this by way of smooth approximating densities. In the treatment of smooth or discontinuous densities it will always be assumed that each streamline (i.e., integral curve for $\mathbf{q}$ ) is a simple Jordan arc connecting $x=-\infty$ to $x=+\infty$. In particular, there are to be no "internal eddies," that is, no relatively compact regions in $S$ bounded by parts of streamlines.

From (2.9) and (2.10) it follows that $\mathbf{q}$ admits a stream function, as does $\mathbf{q}$ multiplied by any function of $\rho$, since $\rho$ is locally constant on streamlines for $\mathbf{q}$. In particular, there is a "pseudo-stream-function" $\psi(x, y)$ for which

$$
\rho^{1 / 2} \mathbf{q}=\left(\psi_{y},-\psi_{x}\right)
$$

the subscripts denoting partial derivatives. The total head pressure $H$ is defined by

$$
H(x, y)=p(x, y)+\frac{1}{2} \rho(x, y)|\mathbf{q}(x, y)|^{2}+\rho(x, y) g y
$$

and from (2.8) it follows that

$$
\partial H / \partial x=\rho V\left(V_{x}-U_{y}\right)+\rho_{x}\left(\frac{1}{2}|\mathbf{q}|^{2}+g y\right)
$$

and

$$
\partial H / \partial y=\rho U\left(U_{y}-V_{x}\right)+\rho_{y}\left(\frac{1}{2}|\mathbf{q}|^{2}+g y\right) .
$$

Since $\mathbf{q} \cdot \nabla \rho=0$, the Bernoulli condition

$$
\mathbf{q} \cdot \nabla H=0
$$

follows. Thus, $H$ and $\rho$ may be considered as functions of the single variable $\psi$, and we shall write $H(x, y)$ or $H(\psi)$ and $\rho(x, y)$ or $\rho(\psi)$ when no confusion is possible. Since all streamlines go to infinity where $\rho$ and $\mathbf{q}$ are known and where $p$ can be assumed hydrostatic:

$$
p(y)=-\int_{0}^{y} \rho_{\infty}(s) g d s
$$

the functions $H(\psi)$ and $\rho(\psi)$ can be computed explicitly when $\rho_{\infty}$ and $c$ are given (cf. (2.21), (2.22)). The explicit examples in $[10, \S 6]$, illustrate the computation.

Define

$$
\Psi(y)=c \int_{0}^{y} \sqrt{\rho_{\infty}(s)} d s
$$


the pseudo-stream-function "at infinity." Let $Y(\psi)$ denote the function inverse to $\Psi$ so that $Y(\Psi(y))=y$ for $-h<y<1-h$. Then all along the streamline with value $\psi$, the density has the value

$$
\rho(\psi)=\rho_{\infty}(Y(\psi)),
$$

and for a flow with a pseudo-stream-function $\psi(x, y)$, the density at $(x, y)$ is $\rho(x, y)$ $=\rho_{\infty}(Y(\psi(x, y)))$. That is, the streamline through $(x, y)$ must be followed to $\infty$ to ascertain its associated density. Similarly, from the data at infinity we have

$$
H(\psi)=p(Y(\psi))+\rho(\psi)\left\{c^{2} / 2+g Y(\psi)\right\},
$$

and each of (2.21) and (2.22) is defined for $\Psi(-h) \leq \psi \leq \Psi(1-h)$.

To obtain a partial differential equation for $\psi$, suppose $U \neq 0$ (as we shall). Using the chain rule to express the derivatives of $H$ and $\rho$ in (2.17), one obtains

$$
\frac{d H}{d \psi}=\sqrt{\rho}\left(U_{y}-V_{x}\right)+\frac{d \rho}{d \psi}\left(\frac{1}{2}|\mathbf{q}|^{2}+g y\right)
$$

after cancelling a factor $\sqrt{\rho} U=\partial \psi / \partial y$. If $U_{y}-V_{x}$ is evaluated using (2.14), the last equation becomes the semilinear elliptic equation

$$
\Delta \psi+g y \frac{d \rho}{d \psi}=\frac{d H}{d \psi}
$$

which is Yih's version of Long's equation (cf. [10] for references). The associated boundary conditions are

$$
\psi(x,-h)=\Psi(-h), \quad \psi(x, 1-h)=\Psi(1-h), \quad x \in R,
$$

and

$$
\lim _{|x| \rightarrow \infty} \psi(x, y)=\Psi(y) .
$$

The formulation at this stage, given $\rho_{\infty}(y)$, is to find $c$ and a function $\psi(x, y)$ satisfying (2.23)-(2.25). If $\rho_{\infty}$ is smooth, one seeks a smooth $\psi$. If $\rho_{\infty}$ is the piecewise constant function

$$
\rho_{d}(y)=\left\{\begin{array}{lc}
\rho_{-}, & -h<y \leq 0 \\
\rho_{+}, & 0<y<1-h,
\end{array}\right.
$$

then the meaning of (2.23) must be clarified. The dividing streamline between the portions of fluid with different densities is that on which $\psi=0$. Suppose it is the graph of a function $\Gamma=\{(x, \gamma(x)) \mid-\infty<x<\infty\}$. In each of the regions $S^{-}=\{(x, y) \mid-h<y<\gamma(x)\}$ and $S^{+}=\{(x, y) \mid \gamma(x)<y<1-h\}, \rho$ is constant, taking the values $\rho^{-}$and $\rho^{+}$, respectively. From (2.19) and (2.22) $H$ is constant in each region and by calculating its values at $x=\infty$ and $y=0$; on either side of $\Gamma$, one sees the values to be $H^{-}=\rho_{-} c^{2} / 2$ and $H^{+}=\rho_{+} c^{2} / 2$, respectively. Equation (2.23) requires $\psi$ to be harmonic in $S^{ \pm}$, as expected. As $\psi$ is a measure of flux, we require it to be continuous. Thus

$$
\psi=0 \text { on } \Gamma
$$

and since it should also satisfy (2.24), by the maximum principle it will be positive in $S^{+}$and negative in $S^{-}$. Let $\psi^{ \pm}$denote the restrictions to $S^{ \pm}$, respectively. An additional requirement on the free boundary $\Gamma$ is that the pressure

$$
p=H-\frac{1}{2}|\nabla \psi|^{2}-\rho g y
$$


be continuous. Thus

$$
\frac{1}{2}\left\{\left|\nabla \psi^{+}\right|^{2}-\left|\nabla \psi^{-}\right|^{2}\right\}=\left(\rho_{+}-\rho_{-}\right)\left\{c^{2} / 2-g y\right\} \quad \text { on } \Gamma,
$$

using the values $H^{ \pm}$from above. To summarize, the boundary-value problem for the given discontinuous density $(2.26)$ is to find $\Gamma, c$, and $\psi(x, y)$ such that $\psi$ is harmonic in $S^{ \pm}$and satisfies (2.24), (2.25), (2.27), and (2.28). There will be no problem interpreting the conditions as it will be seen that $\Gamma$ is smooth as are the extensions of $\psi^{ \pm}$to the closures $\overline{S^{ \pm}}$.

Next we give alternate formulations of the boundary-value problems corresponding to the smooth and discontinuous densities, respectively. We shall work only with flows in which no reversal occurs; that is, for which $U(x, y)>0$ in $\bar{S}$. From (2.14) this corresponds to $\psi_{y}>0$ and for such functions one can solve for $y$ as a function of the spatial coordinate $x$ and the "material coordinate" $\psi$. The utility of this semi-Lagrangian description is that for the density (2.26) the unknown interface function $\gamma$ is merely the unknown function $y(x, \psi)$ evaluated on $\psi=0$; i.e.

$$
\gamma(x)=y(x, 0) \text {. }
$$

A disadvantage of using $y$ as a dependent variable is that the semilinear equation (2.23) is replaced by a singular quasilinear equation for $y(x, \psi)$. Since $y(x, \psi(x, y))$ $=y$, one has the relations

$$
y_{x}+y_{\psi} \psi_{x}=0, \quad y_{\psi} \cdot \psi_{y}=1
$$

from which one derives the equation

$$
-\frac{\partial}{\partial x}\left(\frac{y_{x}}{y_{\psi}}\right)+\frac{1}{2} \frac{\partial}{\partial \psi}\left(\frac{1+y_{x}^{2}}{y_{\psi}}\right)+g y \frac{d \rho}{d \psi}=\frac{d H}{d \psi}
$$

in $\mathbf{R} \times(\Psi(-h), \Psi(1-h))$ from (2.23). The associated boundary conditions are

$$
y(x, \Psi(-h))=-h, \quad y(x, \Psi(1-h))=1-h, \quad x \in \mathbf{R},
$$

and

$$
\lim _{|x| \rightarrow \infty} y(x, \psi)=Y(\psi)
$$

One final change of variables will bring the equation to the form used by the second author in [26]. Let $x_{1}=x, x_{2}=Y(\psi)$, and

$$
w\left(x_{1}, x_{2}\right)=y\left(x_{1}, \Psi\left(x_{2}\right)\right)-x_{2}
$$

so that $w$ represents the deviation at a horizontal position $x_{1}$ of the streamline which has height $x_{2}$ at $\infty$. It should be emphasized that $x_{2}$ is a rescaled stream coordinate. In describing an equation for $w$ we set

$$
f\left(p_{1}, p_{2}\right)=\frac{p_{1}^{2}+p_{2}^{2}}{2\left(1+p_{2}\right)}
$$

$f_{i}=\partial f / \partial p_{i} ; i=1,2 ; \lambda=g / c^{2} ; T=\mathbf{R} \times(-h, 1-h) ;$ and use the summation convention. A calculation produces an eigenvalue problem for the pair $(\lambda, w)$ :

$$
\begin{gathered}
-\left(\partial / \partial x_{i}\right) \rho_{\infty}\left(x_{2}\right) f_{i}(\nabla w)=-\lambda \rho_{\infty}^{\prime}\left(x_{2}\right) w \quad \text { in } T, \\
w\left(x_{1},-h\right)=w\left(x_{1}, 1-h\right)=0, \quad x_{1} \in \mathbf{R},
\end{gathered}
$$




$$
\lim _{|x| \rightarrow \infty} w\left(x_{1}, x_{2}\right)=0 .
$$

The case for the discontinuous density (2.26) requires a separate formulation. While $T$ and $S$ coincide, the domain $T^{+}=\left\{\left(x_{1}, x_{2}\right) \in T \mid x_{2}>0\right\}$ is what corresponds to $S^{+}$. One defines $T^{-}$similarly. By a solution of $(2.36)-(2.38)$ for the density (2.26) we understand a function $w \in C^{0,1}(\bar{T}) \cap C^{1}\left(\overline{T^{ \pm}}\right)$satisfying (2.37), (2.38), and the weak equation

$$
\int_{T} \rho_{d} f_{i}(\nabla w) \frac{\partial \varphi}{\partial x_{i}}=-\lambda \int_{T} \rho_{d}^{\prime} w \varphi=\lambda\left(\rho_{-}-\rho_{+}\right) \int_{-\infty}^{\infty} w\left(x_{1}, 0\right) \varphi\left(x_{1}, 0\right) d x_{1}
$$

for all $\varphi \in C_{0}^{\infty}(T)$. To keep both (2.36) and (2.39) in mind we shall simply refer to a solution of (2.36)-(2.39). Throughout the paper standard notation for function spaces will be used (cf. [13]). We shall write $C$ for a space $C^{0}$ of continuous functions.

2.2 Results.

THEOREM 2.1. Let $\rho_{d}$ be the discontinuous density given in (2.26), let

$$
\lambda_{d}=\frac{1}{\left(\rho_{-}-\rho_{+}\right)}\left(\frac{\rho_{-}}{h}+\frac{\rho_{+}}{1-h}\right),
$$

and let

$$
e=\frac{\rho_{-}}{h^{2}}-\frac{\rho_{+}}{(1-h)^{2}}
$$

Then if $e>0$,

(a) there exists an unbounded, closed, connected set $S \subset \mathbf{R} \times\left(H_{0}^{1}(T) \cap C^{0,1}(\bar{T})\right)$ of solutions $(\lambda, w)$ of $(2.36)-(2.39)$ with $\left(\lambda_{d}, 0\right) \in S$.

(b) There is a positive constant $\underline{\lambda}=\underline{\lambda}\left(\rho_{-}, \rho_{+}\right)$such that if $(\lambda, w) \in S \backslash\left\{\left(\lambda_{d}, 0\right)\right\}$, then $\underline{\lambda} \leq \lambda<\lambda_{d}, w>0$ on $T, w\left(-x_{1}, x_{2}\right)=w\left(x_{1}, x_{2}\right)$ on $T$, and $\partial w / \partial x_{1}<0$ on $(0, \infty) \times(-h, 1-h)$.

(c) The function $w$ has real-analytic extensions to $\overline{T^{+}}$and $\overline{T^{-}}, \partial w / \partial x_{1} \in C^{\alpha}(\bar{T})$, and

$$
\rho_{d}\left(x_{2}\right)\left\{f_{2}(\nabla w)-\lambda w\right\} \in C^{\alpha}(\bar{T})
$$

for some Hölder exponent $\alpha>0$, uniformly for $w$ in bounded sets of $C^{0,1}(\bar{T})$.

(d) There exists a constant $K=K\left(\rho_{-}, \rho_{+}, h\right)$ such that for all $(\lambda, w) \in S$,

$$
0<1 /\left(1+w_{x_{2}}\right) \leq K \quad \text { in } T
$$

and

$$
\left|w_{x_{1}} /\left(1+w_{x_{2}}\right)\right| \leq K \quad \text { in } T .
$$

(e) For each $(\lambda, w) \in S \backslash\left\{\left(\lambda_{d}, 0\right)\right\}$,

$$
\left|w\left(x_{1}, x_{2}\right)\right|+\left|\nabla w\left(x_{1}, x_{2}\right)\right| \leq C_{1} e^{-C_{2}\left|x_{1}\right|}
$$

where $C_{1}$ and $C_{2}$ are positive constants depending on $(\lambda, w)$.

(f) If $\left|\lambda-\lambda_{d}\right|+|w|_{H_{0}^{1}(T)}+|w|_{C^{0,1}(\bar{T})}$ is sufficiently small, the only solution of (2.36)-(2.39) for which $w$ is even in $x_{1}$ and for which both $w$ and $-\partial w / \partial x$ have signs opposite to that of $e$ for $x_{1}>0$ is the trivial solution. 
If $e<0$, the results (a)-(f) hold with the change $w<0$ in $T$ and $\partial w / \partial x_{1}>0$ for $x_{1}>0$ in part (b).

We shall restrict attention to proving parts (a)-(f) for $e>0$ since the case $e<0$ is analogous.

The implications of the assertions in the theorem for the fluid flow are not all transparent and some discussion is in order. The value $\lambda_{d}$ at which bifurcation takes place and the sign of $e$ as an indicator of the direction of streamline displacements both arise in the small-amplitude calculations of Long [16] and Benjamin [8] as well as in the variational approach of the second author in [26]. In the passage from the physical flow model to the problem (2.36)-(2.39) we did not address the question of which solutions $(\lambda, w)$ give rise to physically reasonable flows. In the approaches using the equation (1.23) for $\psi(x, y)$ further restrictions intervene (cf. [4, 10]). Here, however, each pair $(\lambda, w) \in S$ corresponds to a physical flow. The main point is to verify that all streamlines connect $x=-\infty$ to $x=+\infty$ or, equivalently, that $-h<x_{2}+w\left(x_{1}, x_{2}\right)<1-h$ for $-h<x_{2}<1-h$. Since $w=0$ for $x_{2}=-h$ and $x_{2}=1-h$ and since $1+w_{x_{2}}>0$ by part (d), it follows that $x_{2}+w$ lies in the desired range. The bounds in part (d) are, in reality, bounds on velocities, for the use of the relations (2.30) together with (2.14) and (2.34) yields

$$
U=\frac{1}{\sqrt{\rho}} \frac{\partial \psi}{\partial y}=\frac{c}{1+w_{x_{2}}}, \quad V=-\frac{1}{\sqrt{\rho}} \frac{\partial \psi}{\partial x}=\frac{c w_{x_{1}}}{1+w_{x_{2}}}
$$

The velocity $c$ satisfies

$$
\left(g / \lambda_{d}\right)^{1 / 2}<c \leq(g / \underline{\lambda})^{1 / 2}
$$

by part (b), and thus $U$ and $V$ have bounds depending only on $\rho_{-}, \rho_{+}$, and $h$. The velocity $\left(g / \lambda_{d}\right)^{1 / 2}$ is the so-called "critical velocity" associated with the fluid system, and (2.44) shows that all solitary waves found here have supercritical velocities. The remaining parts of (b) describing the shape of $w$ are self-explanatory and are in accord with observed phenomena (cf. [30]). The smoothness of $w$ is more than sufficient to give a sense to (2.36)-(2.39). The condition (2.42) corresponds to the pressure condition (2.28). To see this, one uses $(2.43)$ to obtain

$$
\begin{aligned}
\rho_{\infty}\left\{f_{2}(\nabla w)-\lambda w\right\} & =\rho_{\infty}\left\{\frac{w_{x_{2}}+\frac{1}{2} w_{x_{2}}^{2}-\frac{1}{2} w_{x_{1}}^{2}}{\left(1+w_{x_{2}}\right)^{2}}-\frac{g}{c^{2}} w\right\} \\
& =\frac{1}{c^{2}}\left\{\rho_{\infty}\left(\frac{c^{2}}{2}-g w\right)-\frac{|\nabla \psi|^{2}}{2}\right\} .
\end{aligned}
$$

On the dividing streamline $w\left(x_{1}, 0\right)=y\left(x_{1}, 0\right)$, and the continuity of the expression in (2.45) yields the continuity of pressure expressed by (2.28). With $w$ having the regularity stated in part (c), it is a simple matter to verify that (2.36) holds in $T^{+} \cup T^{-}$and that the weak equation (2.39) holds. The exponential decay described in part (e) shows that the wave is of essentially finite extent. Of course, the proof makes it evident that the constant $C_{2}$ approaches 0 as $\lambda$ approaches $\lambda_{d}$, and this is to be expected in solitary wave phenomena (cf. $[6,8,12,25])$. The nonexistence result in (f) exhibits the delicate nature of the bifurcation at $\left(\lambda_{d}, 0\right)$, a behavior quite different from that at a simple eigenvalue wherein the bifurcating branch crosses the line of trivial solutions. 
3. Solutions for smooth densities. Let $\rho_{n}, n=1,2, \ldots$, be a sequence of nonincreasing functions in $C^{\infty}[-h, 1-h]$ which converge as $n \rightarrow \infty$ to the discontinuous density $\rho_{d}$ given in $(2.26)$. For definiteness suppose that $\rho_{n}=\rho_{-}$ for $-h \leq y \leq 0$ and that $\rho_{n}=\rho_{+}$for $1 / n \leq y \leq 1-h$. Let $\rho_{n}$ play the role of the smooth density $\rho_{\infty}$ in $(2.20)$, and consider solutions $(\lambda, \psi), \lambda=g / c^{2}$, of the corresponding problem $(2.23)-(2.25)$. Let $u=u(x, y)$ be defined by the relation

$$
c \sqrt{\rho_{n}(y)} u(x, y)=\Psi(y)-\psi(x, y) \text {. }
$$

To avoid introducing more notation, we will refer to the pairs $(\lambda, u)$ as solutions of (2.23)-(2.25). In an earlier paper [4] the first author obtained global solution branches for the flow problem with a smooth density, and those results will be used here. The description involves a parameter $\mu_{n}$ defined as follows. Hereafter let $I$ denote the interval $(-h, 1-h)$ and define

$$
\frac{1}{\mu_{n}}=\max _{v \in H_{0}^{1}(I)} \frac{-\int_{I} \rho_{n}^{\prime} v^{2}}{\int_{I} \rho_{n}\left(v^{\prime}\right)^{2}} .
$$

For the smooth density $\rho_{n}$ the quantity $\mu_{n}$ plays the same role that $\lambda_{d}$ in $(2.40)$ plays for the discontinuous density. That is, $\left(\mu_{n}, 0\right)$ is the bifurcation point of a branch of solutions. It will be shown in Lemma 5.5(c) that

$$
\lim _{n \rightarrow \infty} \mu_{n}=\lambda_{d}
$$

Corresponding to $e$ defined in (2.2) is a parameter $A_{n}$ (cf. [4, formula (7.25)]) which satisfies

$$
\lim _{n \rightarrow \infty} A_{n}=e .
$$

For $A_{n}>0$ one obtains waves of elevation for the smooth density $\rho_{n}$, and that is the case discussed here. In $\S 7.2$ of $[4]$ it is shown that there is a maximal, connected set $D_{n} \subset \mathbf{R} \times\left(H_{0}^{1}(S) \cap C(\bar{S})\right)$ of solutions $(\lambda, u)$ containing $\left(\mu_{n}, 0\right)$. Furthermore, for $(\lambda, u) \in D_{n} \backslash\left\{\left(\mu_{n}, 0\right)\right\}$ the parameter $\lambda$ satisfies $0<\gamma_{n} \leq \lambda<\mu_{n}$, where $\gamma_{n}$ is a constant, and the function $u$ satisfies $u>0$ in $S, u(-x, y)=u(x, y)$ in $S$, and $\partial u / \partial x<0$ for $x>0$.

For our purposes the nonlinearity in the basic equation (1.1) of [4] can be assumed smooth and bounded for a fixed smooth density $\rho_{n}$ (cf. the discussion following 7.14 in [4]). Standard elliptic theory applied to such an equation gives local $H^{2}$ bounds from local $H^{1}$ (or $L^{2}$ ) bounds and by embedding theorems, local Hölder estimates (cf. [22]). Hence, if a sequence of solutions converges in $H_{0}^{1}(S)$, it will converge uniformly on bounded subsets of $S$, according to the Arzela-Ascoli theorem. Since $u$ varies monotonically for $x>0$, the argument given in Lemma 4.6 of this paper shows that $u$ converges to zero as $x \rightarrow++\infty$, uniformly on bounded subsets of $H_{0}^{1}(S)$. As a consequence the topology of $\mathbf{R} \times H_{0}^{1}(S)$ is stronger than, and hence equal to, that of $\mathbf{R} \times\left(H_{0}^{1}(S) \cap C(\bar{S})\right)$ when restricted to the set of solutions $D_{n}$. We conclude that we may replace $H_{0}^{1}(S) \cap C(\bar{S})$ by $H_{0}^{1}(S)$ in the preceding paragraph.

A solution $(\lambda, \psi)$ of $(2.23)$ gives rise to a solution $(\lambda, w)$ of $(2.36)$ provided the strict inequality $\psi_{y}>0$ holds in $\bar{S}$. If $u=0$, then $\vartheta(x, y)=\Psi(y)$, whence $\partial \psi / \partial y=\Psi^{\prime}(y)>0$ in $\bar{S}$. Let

$$
C_{n}=\left\{\text { maximal connected subset of } D_{n} \mid \frac{\partial \psi}{\partial y}>0 \text { in } \bar{S}\right\}
$$


and

$$
\varepsilon_{n}=\left\{(\lambda, w) \mid(\lambda, u) \in C_{n}\right\} .
$$

This defines a one-to-one correspondence between $C_{n}$ and $\varepsilon_{n}$, a set of solutions of (2.36)-(2.39). For nontrivial solutions $(\lambda, u)$ we have $\partial u / \partial x<0$ for $x>0$, whence $\partial w / \partial x_{1}<0$ for $x_{1}>0$ by (2.30), (2.34), and (3.1). Since $w$ is even in $x_{1}$ and vanishes at infinity, it follows that $w>0$ in $T$ if $(\lambda, w) \in \mathcal{E}_{n} \backslash\left\{\left(\mu_{n}, 0\right)\right\}$.

LEMMA 3.1. The map from $C_{n}$ to $\mathcal{E}_{n}$ taking $(\lambda, u)$ to $(\lambda, w)$ is one-to-one and continuous from $\mathbf{R} \times H_{0}^{1}(S)$ to $\mathbf{R} \times H_{0}^{1}(T)$. For any rectangle $B=J \times I, J$ open (and possibly unbounded) in $\mathbf{R}$,

$$
|w|_{H^{1}(B)} \leq K_{1}|u|_{H^{1}(B)}
$$

and

$$
|u|_{H^{1}(B)} \leq K_{2}|w|_{H^{1}(B)},
$$

where $K_{1}$ and $K_{2}$ depend on $n$ and $K_{1}$ also depends on a lower bound for $\psi_{y}, \psi$ being associated with $u$. The constants $K_{1}$ and $K_{2}$ are independent of the horizontal interval $J$.

PROOF. The assertions of the lemma are valid for any smooth density, and in the proof we write $\rho$ for $\rho_{n}$, supposing we have fixed $n$. For any pair $(\lambda, u) \in C_{n}$ the corresponding $\psi_{y}>0$, and hence the correspondence between $u$ and $w$, via $y(x, \psi)$, is well defined and one-to-one. A further consequence of $\psi_{y}>0$ is the bound $\Psi(-h) \leq \psi \leq \Psi(1-h)$ in view of the boundary conditions (2.24). This bound, together with standard elliptic estimates applied to the semilinear equation (2.23), yields $L^{\infty}$ estimates for derivatives of $\psi$ of all orders, depending only on $\rho$ (that is, on $n$ ). In particular $\psi_{y} \leq K$, where $K$ depends on $n$.

In comparing the $H^{1}$ norms of $u$ and $w$, we first note that since $\rho$ is smooth and positive and the speed $c=(g / \lambda)^{1 / 2}$ is bounded above and below on $C_{n}$, it suffices to prove inequalities (3.7) and (3.8) with $u$ replaced by

$$
\varphi(x, y)=c \sqrt{\rho(y)} u(x, y)=\Psi(y)-\psi(x, y) .
$$

From $(2.20),(2.30)$ and the coordinate relation (2.34) one obtains

$$
\begin{aligned}
\frac{\partial w}{\partial x_{2}} & =\frac{\partial y}{\partial \psi}\left(x, \Psi\left(x_{2}\right)\right) \frac{\partial \Psi}{\partial x_{2}}\left(x_{2}\right)-1 \\
& =\frac{c \sqrt{\rho\left(x_{2}\right)}}{\partial \psi\left(x, y\left(x, \Psi\left(x_{2}\right)\right)\right) / \partial y}-1 \\
& =\frac{c \sqrt{\rho(Y(\psi(x, y)))}}{\partial \psi(x, y) / \partial y}-1
\end{aligned}
$$

and

$$
\frac{\partial w}{\partial x_{1}}=\frac{\partial y}{\partial x}=-\frac{\partial \psi(x, y) / \partial x}{\partial \psi(x, y) / \partial y}
$$

It follows that, for each fixed $x=x_{1}$,

$$
\int_{I}|\nabla w|^{2} d x_{2}=\int_{I} \frac{1}{\psi_{y} c \sqrt{\rho(Y(\psi))}}\left\{\psi_{x}^{2}+\left(c \sqrt{\rho(Y(\psi))}-\psi_{y}\right)^{2}\right\} d y .
$$


For a wave of elevation $w>0$ and $\psi(x, y)<\Psi(y)$, and so $\rho(Y(\psi(x, y))) \geq$ $\rho(Y(\Psi(y)))=\rho(y)$. Thus

$$
\begin{aligned}
c \int_{-h}^{y} & \sqrt{\rho(Y(\psi(x, s)))} d s+\Psi(-h)-\psi(x, y) \\
& \geq c \int_{-h}^{y} \sqrt{\rho(s)} d s+\int_{0}^{-h} c \sqrt{\rho(s)} d s-\psi(x, y) \\
& =c \int_{0}^{y} \sqrt{\rho(s)} d s-\psi(x, y) \\
& =\Psi(y)-\psi(x, y)
\end{aligned}
$$

in view of the definition (2.20). Since $c \int_{-h}^{y} \sqrt{\rho(Y(\psi))}+\Psi(-h)-\psi(x, y)$ vanishes at $y=-h,(3.13)$ can be combined with the Poincaré inequality to yield

$$
\begin{aligned}
\int_{I} \varphi^{2}(x, y) d y & =\int_{I}(\Psi(y)-\psi(x, y))^{2} \\
& \leq \int_{I}\left(\int_{-h}^{y} c \sqrt{\rho(Y(\psi(x, s))} d s+\Psi(-h)-\psi\right)^{2} \\
& \leq \int_{I}\left(\frac{\partial}{\partial y}\left(\int_{-h}^{y} c \sqrt{\rho(Y(\psi))}+\Psi(-h)-\psi\right)\right)^{2} \\
& =\int_{I}\left(c \sqrt{\rho(Y(\psi))}-\psi_{y}\right)^{2} .
\end{aligned}
$$

Since $\psi_{y} \leq K$ it follows from (3.12) and (3.14) that, for any rectangle $B=[\alpha, \beta] \times I$,

$$
\int_{B} \varphi^{2} \leq K^{\prime} \int_{B}|\nabla w|^{2} \leq K^{\prime}|w|_{H^{1}(B)}^{2},
$$

where one uses the bounds on $\psi_{y}$ and $c$ in the denominator in (3.12). Since $\psi_{x}=\varphi_{x}$, (3.12) also yields

$$
\begin{aligned}
K^{\prime} \int_{B}|\nabla w|^{2} & \geq \int_{B}\left\{\varphi_{x}^{2}+\left(c \sqrt{\rho Y(\psi)}-c \sqrt{\rho Y(\Psi)}+c \sqrt{\rho Y(\Psi)}-\psi_{y}\right)^{2}\right\} \\
& \geq \int_{B}\left\{\varphi_{x}^{2}+\frac{1}{2} \varphi_{y}^{2}-K^{\prime \prime} \varphi^{2}\right\},
\end{aligned}
$$

where the mean value theorem is used to obtain

$$
|\sqrt{\rho Y(\psi)}-\sqrt{\rho Y(\Psi)}|^{2} \leq K^{\prime \prime}|\psi-\Psi|^{2}
$$

From (3.15) and (3.16) it is clear that

$$
|\varphi|_{H^{1}(B)} \leq K_{0}|w|_{H^{1}(B)}
$$

and hence (3.8) holds.

For a fixed $(\lambda, \psi) \in C_{n}$, there holds $\psi_{y} \geq K^{\prime}>0$ and one readily derives

$$
|w|_{H^{1}(B)} \leq K_{1}^{\prime}|\varphi|_{H^{1}(B)}
$$

from (3.12) using a mean value estimate as in (3.16). Hence (3.7) holds.

To see continuity of the map from $C_{n}$ to $\mathcal{E}_{n}$, consider a sequence of solutions $\left(\lambda_{m}, u_{m}\right), m=1,2, \ldots$, converging in $\mathbf{R} \times H_{0}^{1}(S)$ to $(\lambda, u) \in \mathcal{C}_{n}$. Let $w_{m}$ and $w$ 
be the respective counterparts of $u_{m}$ and $u$. If $\lambda=\mu_{n}$, then $u=0$ according to the description of $D_{n}$. Then since $u_{m}$ converges to zero in $H_{0}^{1}(S)$, elliptic estimates show $\nabla u_{m}$ to be small uniformly on $S$ for all large $m$. The $y$ derivatives of the corresponding $\psi_{m}$ are bounded below by a positive constant, uniformly for $m$ large, and hence (3.7) implies the convergence of $w_{m}$ to zero in $H_{0}^{1}(S)$.

If $\lambda<\mu_{n}$, then $\lambda_{m}<\left(\lambda+\mu_{n}\right) / 2<\mu_{n}$ for all large $m$ and the function $\psi$ corresponding to $u$ satisfies $\partial \psi / \partial y>0$ in $\bar{S}$. In fact, there is a constant $K>0$ so that $\partial \psi / \partial y \geq K$ on $\bar{S}$ and $\partial \psi_{m} / \partial y \geq K$ on $\bar{S}$ for all sufficiently large $m$. To see this, consider first the behavior for large $x$. The method of proof of Theorem 2.3 (b) of [4] is easily used to show that $\left|u_{m}\right| \leq K^{\prime} e^{-\beta|x|}$ and $|u| \leq K^{\prime} e^{-\beta|x|}$, where $\beta \geq K^{\prime \prime}\left(\mu_{n}-\lambda\right)$. Elliptic estimates show that $\nabla u_{m}$ and $\nabla u$ have the same type of decay for large $x$. Let $B_{N}$ denote the subset of $S$ (or $T$ ) where $|x| \leq N$. It follows from (3.1) and the exponential decay that for some $N>0$ and all $m$ sufficiently large the $y$ derivatives of $\psi$ and $\psi_{m}$ are bounded below by a constant $K^{\prime \prime \prime}>0$. As noted at the outset of the proof, the second derivatives of the function $\psi_{m}$ have a bound on $\bar{S}$, independent of $m$. By the Arzela-Ascoli theorem it can be assumed that the functions $\partial \psi_{m} / \partial y$ converge uniformly to $\partial \psi / \partial y$ on $B_{N}$. Since $\partial \psi / \partial y>0$ on $\bar{B}_{N}$, it is clear that for all sufficiently large $m$ there is a positive $K$ which serves on $\bar{B}_{N}$ and on its complement as a lower bound for the $y$ derivatives of $\psi$ and $\psi_{m}$.

To see that $w_{m}$ converges to $w$, first note that, according to the lower bound just estabished, (3.7) holds for the pair $(u, w)$ and for the pairs $\left(u_{m}, w_{m}\right)$ with $K$ independent of $m$, provided $m$ is sufficiently large. For any given $\varepsilon>0$ the norm of $u$ in $H^{1}\left(S-B_{N}\right)$ will be less than $\varepsilon$ for $N$ sufficiently large, and the norm of $u_{m}$ in $H^{1}\left(S-B_{N}\right)$ will be less than $2 \varepsilon$ for $m$ sufficiently large. Then the norm of $w_{m}$ in $H^{1}\left(T-B_{N}\right)$ will be at most $2 K_{1} \varepsilon$, and so it suffices to show that $w_{m}$ converges to $w$ in $H^{1}\left(B_{N}\right)$ for each $N$. Since the $y$ derivatives of the $\psi_{m}$ have a positive lower bound $K$ for all large $m$, one readily sees that the inverse functions $y_{m}(x, \psi)$ converge uniformly to $y(x, \psi)$ on $B_{N}$ as do the functions $w_{m}$ to $w$ on $B_{N}$. Derivatives of the formulas (3.10) and (3.11) provide $C^{2}$ bounds for the functions $w_{m}$, uniformly in $m$ for all sufficiently large $m$, and hence another use of the Arzela-Ascoli theorem gives convergence of $w_{m}$ to $w$ in $C^{1}\left(B_{N}\right)$ and hence in $H^{1}\left(B_{N}\right)$. Q.E.D.

THEOREM 3.2. For each $n, \mathcal{E}_{n}$ is an unbounded, closed, connected subset of $\mathbf{R} \times H_{0}^{1}(T)$ containing $\left(\mu_{n}, 0\right)$.

ProOF. The continuity of the map from $C_{n}$ to $\mathcal{E}_{n}$ implies that $\mathcal{E}_{n}$ is connected. To see that $\mathcal{E}_{n}$ is closed, let $\left(\lambda_{m}, w_{m}\right) \in \mathcal{E}_{n}, m=1,2, \ldots$, converge to $(\lambda, w)$ in $\mathbf{R} \times H_{0}^{1}(T)$. The inequality (3.8) shows that the corresponding functions $u_{m}$ are uniformly bounded in $H_{0}^{1}(S)$. From $\S 5$ of [4] it follows that the sequence $\left(\lambda_{m}, u_{m}\right)$ converges in $\mathbf{R} \times H_{0}^{1}(S)$ to a solution $(\lambda, u)$. The function $u$ will be smooth as will the corresponding $\psi$, again from elliptic estimates. In order to show that $\mathcal{E}_{n}$ is closed, it suffices to show that $\partial \psi / \partial y>0$ in $\bar{S}$.

If $u \equiv 0$, then $\psi=\Psi$ and its $y$ derivative is positive in $\bar{S}$. If $u \not \equiv 0$, then $\lambda<\mu_{n}$ and $\nabla u$ decays exponentially to zero as $|x| \rightarrow \infty$ as was indicated in the previous proof. This decay means that $\partial \psi / \partial y$ is positive for $(x, y) \in \bar{S} \backslash B_{N}$, for some $N$. Since $\rho$ is constant near $y=-h$ and near $y=1-h, \psi$ is harmonic near those lines. Moreover, $\psi$ is nonconstant near those lines, for otherwise it could not approach $\Psi$ 
as $|x| \rightarrow \infty$. From the maximum principle it follows that $\partial \psi / \partial y$ is positive on the boundary $\partial S$ and so if it vanishes, it must do so at a point $(\tilde{x}, \tilde{y}) \in S$. Suppose this occurs. Then since $\partial \psi / \partial y \geq 0$ in $S$, the gradient of $\partial \psi / \partial y$ must vanish at $(\tilde{x}, \tilde{y})$ with the result that

$$
0 \leq \partial \psi / \partial y<K^{\prime}\left((x-\tilde{x})^{2}+(y-\tilde{y})^{2}\right)
$$

in a neighborhood of $(\tilde{x}, \tilde{y})$. But then $\left(\psi_{y}\right)^{-1}$ has a nonintegrable singularity at $(\tilde{x}, \tilde{y})$. Now apply $(3.12)$ to $w_{m}$ and $\psi_{m}$ and integrate the result over an $x$ interval containing $\tilde{x}$. Since $w_{m}$ is bounded in $H^{1}$ uniformly in $m$, one can conclude that the integral of $\left(\partial \psi_{m} / \partial y\right)^{-1}$ over a neighborhood of $(\tilde{x}, \tilde{y})$ is bounded, uniformly in $m$. Fatou's lemma would then show $(\partial \psi / \partial y)^{-1}$ to be integrable near $(\tilde{x}, \tilde{y})$, a contradiction.

The contradiction just reached also arises if one assumes $\mathcal{E}_{n}$ bounded. For then (3.8) shows that $C_{n}$ is bounded, and hence there is a sequence $\left(\lambda_{m}, u_{m}\right)$ converging to $(\lambda, u) \in D_{n} \backslash C_{n}$ for which the corresponding $\psi_{y}$ must vanish somewhere. Q.E.D. For later use we include the following result.

LEMMA 3.3. For each $n$, the identity map on $\mathcal{E}_{n}$ is continuous from $\mathbf{R} \times H_{0}^{1}(T)$ to $\mathbf{R} \times\left(H_{0}^{1}(T) \cap C^{1}(\bar{T})\right)$.

PROOF. The proof is similar to the proof of continuity in Lemma 3.1, and we use notation from that proof. In fact the continuity on bounded subsets of $T$ follows from the arguments in the last paragraph of that proof and so only the "tail" behavior need be examined. Suppose $\left(\lambda_{m}, w_{m}\right)$ converges to $(\lambda, w)$ in $\mathbf{R} \times H_{0}^{1}(T)$. Then from (3.8) the corresponding $\left|u_{m}\right|_{H^{1}\left(S-B_{N}\right)}$ can be made arbitrarily small for large $N$, uniformly in $m$. Elliptic estimates then show $\left|u_{m}\right|_{C^{1}\left(S-B_{N}\right)}$ to be small, uniformly in $m$. Since the function $\psi$ corresponding to $w$ satisfies $\partial \psi / \partial y \geq K>0$ in $\bar{S}$, one has $\partial \psi_{m} / \partial y \geq K / 2$ in $\bar{S}$ for the corresponding $\psi_{m}$ when $m$ is sufficiently large. Then the formulas (3.9)-(3.11) show $\left|w_{m}\right|_{C^{1}\left(S-B_{N}\right)}$ to be small uniformly in $m$, so the identity map is continuous. Q.E.D.

4. Sets of solutions with restricted gradients. In $\S 3$ it was shown that for each $n$ the problem

$$
\begin{gathered}
-\left(\partial / \partial x_{i}\right)\left(\rho_{n}\left(x_{2}\right) f_{i}(\nabla w)\right)=-\lambda \rho_{n}^{\prime}\left(x_{2}\right) w \quad \text { in } T, \\
w\left(x_{1},-h\right)=w\left(x_{1}, 1-h\right)=0, \quad x_{1} \in \mathbf{R}, \\
\lim _{\left|x_{1}\right| \rightarrow \infty} w\left(x_{1}, x_{2}\right)=0
\end{gathered}
$$

has an unbounded, closed, connected set of solutions $\mathcal{E}_{n}$ emanating from the pair $\left(\mu_{n}, 0\right)$. As stated earlier, the existence of solutions for the problem with discontinuous density will be obtained by allowing $n$ to approach infinity. The intricacies of the limiting process will be described in $\S 5$. Here we describe a general setting for obtaining connected sets of solutions by letting parameters approach limits and also prove some estimates which will be used in the limiting process.

Consider a metric space $X$ and a collection of sets $A=\left\{A_{n}\right\}, n=1,2, \ldots$, with $A_{n} \subset X$ for each $n$. By definition liminf $A$ consists of points $p \in X$ such that every neighborhood of $p$ contains points of all but a finite number of the sets $A_{n}$ while lim sup $A$ consists of those $p \in X$ such that every neighborhood of $p$ contains points from infinitely many of the sets $A_{n}$. The following result from Whyburn $[31, \mathrm{p}$. 15] provides a tool for demonstrating the permanence of connected sets. 
LEMMA 4.1. Let $\left\{A_{n}\right\}, n=1,2, \ldots$, be a sequence of connected sets in a metric space such that

(a) $\bigcup_{n=1}^{\infty} A_{n}$ is precompact and

(b) $\liminf \left\{A_{n}\right\} \neq \varnothing$.

Then $\lim \sup \left\{A_{n}\right\}$ is a compact, connected set.

While our eventual goal is an unbounded, connected set $S$ in $\mathbf{R} \times\left(H_{0}^{1}(T) \cap\right.$ $C^{0,1}(\bar{T})$ ), a first step will be the application of Lemma 4.1 to bounded sets in $X=\mathbf{R} \times H_{0}^{1}(T)$. The bounded sets to be considered are defined as follows. For $\delta \in(0,1)$ and $R>0$ let

$$
B(R)=\left\{(\lambda, w) \in \mathbf{R} \times\left. H_{0}^{1}(T)|| w\right|_{H_{0}^{1}} \leq R \text { and } 0 \leq \lambda \leq 2 \lambda_{d}\right\}
$$

and

$$
Q_{\delta}=\left\{\left(p_{1}, p_{2}\right) \in \mathbf{R}^{2} \mid p_{1} \in\left[-\delta^{-1}, \delta^{-1}\right], p_{2} \in\left[-1+\delta, \delta^{-1}\right]\right\} .
$$

Starting with $\varepsilon_{n}$ from the previous section, let

$$
\tilde{F}_{n, \delta, R}=\left\{(\lambda, w) \in \mathcal{E}_{n} \mid(\lambda, w) \in B(R) ; \nabla w\left(x_{1}, x_{2}\right) \in Q_{\delta}, \quad\left(x_{1}, x_{2}\right) \in T\right\}
$$

and

$$
F_{n, \delta, R}=\left\{\text { maximal connected subset of } \tilde{F}_{n, \delta, R} \text { containing }\left(\mu_{n}, 0\right)\right\} \text {. }
$$

Here $\mathcal{E}_{n}$ and $F_{n, \delta, R}$ are regarded as subsets of $\mathbf{R} \times H_{0}^{1}(T)$. For $(\lambda, w) \in \mathcal{E}_{n}, \nabla w$ is smooth and approaches zero as $\left|x_{1}\right| \rightarrow \infty$, so the condition $\nabla w \in Q_{\delta}$ is unambiguous. It will be shown in Lemma 5.5 (c) that $\mu_{n} \rightarrow \lambda_{d}$ as $n \rightarrow \infty$ so that for each pair $(\delta, R), \lim \inf \left\{F_{n, \delta, R}\right\}$ contains $\left(\lambda_{d}, 0\right)$. It may also be assumed that the values of $n$ under consideration are large enough so that $\mu_{n}<2 \lambda_{d}$, making $\left(\mu_{n}, 0\right)$ lie in $B(R)$.

From Theorem $3.2, \mathcal{E}_{n}$ is an unbounded, connected set containing $\left(\mu_{n}, 0\right)$. In $\S 5$ it will be shown that for each $\delta \in(0,1)$ and each $R>0$ the collection $\bigcup_{n} F_{n, \delta, R}$ is precompact. Thus from Lemma 4.1

$$
G_{\delta, R}=\lim \sup \left\{F_{n, \delta, R}\right\}
$$

is a compact, connected set containing $\left(\lambda_{d}, 0\right)$. In $\S 6$ it is shown that $G_{\delta, R}$ is still connected as a subset of $\mathbf{R} \times\left(H_{0}^{1}(T) \cap C^{0,1}(\bar{T})\right)$. Moreover, it is shown that $G_{\delta, R}$ must contain a pair $(\lambda, w)$ for which $|w|_{H_{0}^{1}}=R$ of for which $\max \left\{\left|w_{x_{1}}\right|_{L^{\infty}(T)},\left|w_{x_{2}}\right|_{L^{\infty}(T)}\right\}$ $=\delta^{-1}$. The set

$$
S=\bigcup_{N=2}^{\infty} G_{1 / N, N}
$$

will then be seen to fulfill the requirements of Theorem 2.1.

The restrictions embodied in the definition of $F_{n, \delta, R}$ reflect an intimate link between the topologies in which there are unbounded solution branches and those in which one can do analysis. The solution pairs $(\lambda, w) \in \mathcal{E}_{n}$ will not be unbounded in $\mathbf{R} \times C(\bar{T})$ nor could we hope to analyze equation (4.1) adequately for $w$ having less than $C^{0,1}$ regularity. The singularity of $f\left(p_{1}, p_{2}\right)$ for $p_{2}=-1$ and the lack of uniform convexity for large values of $\left(p_{1}, p_{2}\right)$ make elliptic estimates elusive. The behavior of $\rho_{n}$ compounds the difficulty. For solutions in $F_{n, \delta, R}$, however, one can 
obtain the estimates needed for the delicate compactness arguments carried out in the next section and ultimately used in Lemma 4.1 .

For the remainder of this section it is assumed that $n, \delta$, and $R$ are fixed and estimates are derived for solutions $(\lambda, w) \in F_{n, \delta, R}$. We suppress $n$, writing $\rho$ for $\rho_{n}$. The constants in the estimates may depend on one or more of the parameters $n, \delta$, and $R$ and this will be indicated. Dependence on $\rho_{+}, \rho_{-}$, and $h$ is still suppressed. Recall that for large $n$, the number $\lambda$ is bounded above by $2 \lambda_{d}$ for solutions under consideration. Thus, while the size of $\lambda$ enters in the estimates, it can be absorbed into constants depending only on $\rho_{+}, \rho_{-}$and $h$.

LEMMA 4.2. If $(\lambda, w) \in F_{n, \delta, R}$ and $m \in \mathbf{R}$,

$$
\int_{m}^{m+1} \int_{I}\left|\nabla w_{x_{1}}\right|^{2} \leq K_{1} \int_{m-1}^{m+2} \int_{I}|\nabla w|^{2} \leq K_{2} \int_{m-2}^{m+3} \int_{I} w^{2}
$$

where $K_{1}$ and $K_{2}$ depend on $\delta$. For $\varepsilon>0$ let $I_{\varepsilon}=\left\{x_{2} \in I|| x_{2} \mid>\varepsilon\right\}$. Then

$$
\int_{m}^{m+1} \int_{I_{\varepsilon}}\left|\nabla w_{x_{2}}\right|^{2} \leq K_{3} \int_{m-1}^{m+2} \int_{I}|\nabla w|^{2},
$$

where $K_{3}$ depends on $\delta$ and $\varepsilon$.

PROOF. Let $\varsigma \in C_{0}^{\infty}(\mathbf{R})$ be a function which has range in $[0,1]$, has support in $[m-2, m+3]$, and is 1 for $x_{1} \in[m-1, m+2]$. Multiply equation (4.1) by $\varsigma^{2} w$ and integrate over $T$ to obtain

$$
\frac{1}{2} \int_{T} \rho \varsigma^{2} \frac{2+w_{x_{2}}}{\left(1+w_{x_{2}}\right)^{2}}|\nabla w|^{2}=-2 \int_{T}\left[\rho \zeta \zeta^{\prime} \frac{w w_{x_{1}}}{1+w_{x_{2}}}\right]+2 \lambda \int_{T} \rho \varsigma^{2} w w_{x_{2}}
$$

after an integration by parts. For $\nabla w \in Q_{\delta}$ the expression $\left(2+w_{x_{2}}\right) /\left(1+w_{x_{2}}\right)^{2}$ is bounded below by a positive constant depending on $\delta$. Thus if Young's inequality is used on the right-hand side of (4.11) and a small multiple of $\iint \rho \varsigma^{2}|\nabla w|^{2}$ is subtracted from the left side, the second inequality in (4.9) follows.

Now let $v=w_{x_{1}}$ and $f_{i j}=\partial^{2} f / \partial p_{i} \partial p_{j}$ for $i, j=1,2$. The result of applying $\partial / \partial x_{1}$ to both sides of $(4.1)$ is

$$
-\frac{\partial}{\partial x_{i}} \rho f_{i j}(\nabla w) \frac{\partial v}{\partial x_{j}}=-\lambda \rho^{\prime} v .
$$

Let $\zeta$ denote a new cutoff function which is 1 on $[m, m+1]$ and has support in $[m-1, m+2]$. If (4.12) is multiplied by $\varsigma^{2} v$ and an integration by parts is used, the equation

$$
\int_{T} \rho \varsigma^{2} f_{i j} \frac{\partial v}{\partial x_{i}} \frac{\partial v}{\partial x_{j}}=-2 \int_{T} \rho \zeta \zeta^{\prime} v f_{1 j} \frac{\partial v}{\partial x_{j}}+2 \lambda \int_{T} \rho \varsigma^{2} v v_{x_{2}}
$$

is obtained. A simple calculation shows that the quadratic form determined by $f_{i j}\left(p_{1}, p_{2}\right)$ satisfies

$$
\begin{aligned}
f_{i j} \alpha_{i} \alpha_{j} & =\frac{1}{\left(1+p_{2}\right)}\left\{\alpha_{1}^{2}-\frac{2 p_{1} \alpha_{1} \alpha_{2}}{1+p_{2}}+\frac{\alpha_{2}^{2}\left(1+p_{1}^{2}\right)}{\left(1+p_{2}\right)^{2}}\right\} \\
& \geq \frac{1}{1+p_{2}}\left\{\alpha_{1}^{2}(1-\eta)+\frac{\alpha_{2}^{2}}{\left(1+p_{2}\right)^{2}}\left[1-p_{1}^{2}\left(\frac{1}{\eta}-1\right)\right]\right\}
\end{aligned}
$$


for all $\left(\alpha_{1}, \alpha_{2}\right) \in \mathbf{R}^{2}$ and any $\eta>0$. In $Q_{\delta}, p_{1}^{2}<\delta^{-2}$ and $\delta \leq 1+p_{2} \leq 1+\delta^{-1}$. The choice $\eta^{-1}=1+\delta^{2} / 2$ in (4.14) yields

$$
f_{i j} \alpha_{i} \alpha_{j} \geq \delta^{3}\left(\alpha_{1}^{2}+\alpha_{2}^{2}\right) / 16 \text {. }
$$

Furthermore, for $\left(p_{1}, p_{2}\right) \in Q_{\delta}$,

$$
\left|f_{i j}\right| \leq K / \delta^{5}
$$

and so the use of Young's inequality, as before, yields the first inequality in (4.9). One cannot hope to obtain an inequality just like (4.9) for $w_{x_{2}}$, independently of $n$, for the discontinuity in the limiting density produces a discontinuity in $w_{x_{2}}$ along $x_{2}=0$. However, for $-h<x_{2}<-\varepsilon / 2, \rho$ is constant and $w$ satisfies

$$
\partial f_{i}(\nabla w) / \partial x_{i}=0 .
$$

If $w$ is extended to $-2 h+\varepsilon / 2<x_{2} \leq-h$ so as to be odd with respect to $x_{2}=-h$, it will satisfy (4.17) in the extended region. Suppose now that $\zeta$ is a cutoff function which is 1 on $[m, m+1] \times[-2 h+\varepsilon,-\varepsilon]$ and which has support on $[m-1, m+$ $2] \times[-2 h+\varepsilon / 2,-\varepsilon / 2]$. Let $z\left(x_{1}, x_{2}\right)=w_{x_{2}}\left(x_{1}, x_{2}\right)$. Differentiation of (4.17) with respect to $x_{2}$ produces

$$
\frac{\partial}{\partial x_{i}} f_{i j}(\nabla w) \frac{\partial z}{\partial x_{j}}=0 .
$$

If equation (4.18) is multiplied by $\varsigma^{2} z$ and the pattern of the previous estimates repeated, there results an inequality similar to (4.10) but with the left-hand member integrated over $-h \leq x_{2} \leq-\varepsilon$. The constant $K_{3}$ depends on $\delta$ and on $\varepsilon$ (through the derivative $\left.\partial \varsigma / \partial x_{2}\right)$. The estimate for $x_{2}>\varepsilon$, valid for all $n$ such that $\rho=\rho_{n}$ is constant for $x_{2}>\varepsilon / 2$ (i.e., $n>1 / 2 \varepsilon$ ), is done similarly. Q.E.D.

The next lemma can be obtained using standard elliptic theory as in [26, Lemma 3.2]. However, in the case of two independent variables, Hölder estimates follow from the readily accessible results of Meyers [18], and we choose to use those here.

LEMMA 4.3. Suppose $(\lambda, w) \in F_{n, \delta, R}$. Then there is an $\alpha>0$, depending on $\delta$, such that for any $m \in \mathbf{R}$

$$
\begin{gathered}
|w|_{C^{\alpha}([m, m+1] \times I)}^{2} \leq K_{0} \int_{m-2}^{m+3} \int_{I} w^{2} \\
\left|w_{x_{1}}\right|_{C^{\alpha}([m, m+1] \times I)}^{2} \leq K_{1} \int_{m-2}^{m+3} \int_{I} w^{2}
\end{gathered}
$$

and for $n>1 / 2 \varepsilon$

$$
\left|w_{x_{2}}\right|_{C^{\alpha}\left([m, m+1] \times I_{\varepsilon}\right)}^{2} \leq K_{2} \int_{m-2}^{m+3} \int_{I} w^{2},
$$

where $K_{0}, K_{1}$, and $K_{2}$ depend on $\delta$ and $K_{2}$ depends on $\varepsilon$ as well.

ProOF. The function $w$ satisfies the equation

$$
\frac{\partial}{\partial x_{i}} \rho \tilde{f}_{i j} \frac{\partial w}{\partial x_{j}}=\lambda \rho^{\prime} w
$$


where

$$
\tilde{f}_{i j}\left(x_{1}, x_{2}\right)=\int_{0}^{1} f_{i j}\left(t \nabla w\left(x_{1}, x_{2}\right)\right) d t .
$$

Since $Q_{\delta}$ is convex, $\tilde{f}_{i j}$ satisfies the inequalities (4.15) and (4.16) obtained for $f_{i j}$. Let $\tilde{A}$ be the matrix with components $\rho \tilde{f}_{i j}(i, j=1,2)$, and let $u=\varsigma w$, where $\varsigma$ is a function of $x_{1}$ which has support in $m-1<x_{1}<m+2$ and which is 1 for $m \leq x_{1} \leq m+1$. Let $\Omega$ be a domain with a boundary $\partial \Omega$ of class $C^{\infty}$ satisfying

$$
[m-1, m+2] \times I \subset \Omega \subset[m-2, m+3] \times I .
$$

From (4.22) it follows that

$$
\begin{aligned}
\operatorname{div}(\tilde{A} \operatorname{grad} u)=\operatorname{div} f+g & \text { in } \Omega, \\
u=0 & \text { on } \partial \Omega,
\end{aligned}
$$

where

$$
f=w \tilde{A} \operatorname{grad} \zeta+(0, \lambda \zeta \rho w)
$$

and

$$
g=-\lambda \zeta \rho w_{x_{2}}+\operatorname{grad} \zeta \cdot(\tilde{A} \operatorname{grad} w) .
$$

From Lemma 4.2 and the Sobolev embedding theorem it follows that

$$
|f|_{L^{p}(\Omega)}+|g|_{L^{2}(\Omega)} \leq K_{p}|w|_{L^{2}([m-2, m+3] \times I)}
$$

for any $p \in[2, \infty)$. Since $\tilde{A}$ satisfies a uniform ellipticity condition (cf. (4.15), (4.16)), Theorem 1 of Meyers [18] yields

$$
|\operatorname{grad} u|_{L^{p}(\Omega)} \leq K|w|_{L^{2}([m-2, m+3] \times I)}
$$

for some $p=p(\delta)>2$ and a $K$ depending on $\delta$ and $p(\delta)$. Now from embedding theory (cf. $[13,22])$ the inequality (4.19) follows.

Inequality (4.20) follows in a similar manner by using equation (4.12). One uses $A$ with entries $\rho f_{i j}(\nabla w)$ in place of $\tilde{A}$, lets $f=v A \operatorname{grad} \zeta+(0, \lambda \zeta \rho v)$, and lets $g=-\lambda \zeta \rho v_{x_{2}}+\operatorname{grad} \zeta \cdot A \operatorname{grad} v$. The Sobolev embedding theorem together with Lemma 4.2 (with a cutoff function equal to 1 on $\Omega$ ) yields

$$
|v|_{L^{p}(\Omega)} \leq K|w|_{L^{2}([m-2, m+3] \times I)}
$$

and Meyer's result gives

$$
|\operatorname{grad} v|_{L^{p}(\Omega)} \leq K|w|_{L^{2}([m-2, m+3] \times I)} .
$$

This last inequality implies (4.20) and will also be used in what follows. The proof of (4.21) is done similarly but with a cutoff function $\zeta$ which vanishes for $\left|x_{2}\right|<\varepsilon / 2$. To complete the proof we merely note that while the values of $\alpha$ occurring in the three estimates may differ, we may let $\alpha$ stand for the smallest one. Likewise we let $p=p(\delta)$ stand for the smallest one occurring. Q.E.D.

The estimates obtain so far can be combined with equation (4.1) to produce improved estimates for $w_{x_{2}}$. 
THEOREM 4.4. Suppose $(\lambda, w) \in F_{n, \delta, R}$ and $m \in \mathbf{R}$. Then

$$
\text { (a) }\left(\int_{m-1}^{m} \int_{I}\left|\operatorname{grad}\left\{\rho\left(f_{2}(\nabla w)-\lambda w\right)\right\}\right|^{p}\right)^{2 / p} \leq K_{1} \int_{m-3}^{m+4} \int_{I} w^{2},
$$

where $p=p(\delta)$ from Lemma 4.3 and $K_{1}$ depend on $\delta$.

(b) $\left|\rho\left(f_{2}(\nabla w)-\lambda w\right)\right|_{C^{\alpha}([m, m+1] \times I)}^{2} \leq K_{2} \int_{m-3}^{m+4} \int_{I} w^{2}$,

where $\alpha$ (as in Lemma 4.3) and $K_{2}$ depend on $\delta$.

$$
\text { (c) }\left|w_{x_{2}}\right|_{C([m, m+1] \times I)}^{2} \leq K_{3} \int_{m-3}^{m+4} \int_{I} w^{2},
$$

where $K_{3}$ depends on $\delta$.

(d) If $\rho^{\prime} \equiv 0$ on a convex subset $\Omega$ of $[m, m+1] \times I$, then

$$
\left|w_{x_{2}}\right|_{C^{\alpha}(\Omega)}^{2} \leq K_{4} \int_{m-3}^{m+4} \int_{I} w^{2}
$$

where $\alpha$ is the exponent from part (b) and $K_{4}$ depends on $\delta$.

Proof. For part (a) let $F\left(x_{1}, x_{2}\right)=\rho\left(x_{2}\right)\left\{f_{2}(\nabla w)-\lambda w\right\}$. Recalling the notation $v=w_{x_{1}}$, one has

$$
\partial F / \partial x_{1}=\rho\left\{f_{21}(\nabla w) v_{x_{1}}+f_{22}(\nabla w) v_{x_{2}}-\lambda v\right\}
$$

and from equation (4.1)

$$
\begin{aligned}
-\partial F / \partial x_{2} & =\left(\partial / \partial x_{1}\right)\left(\rho f_{1}(\nabla w)\right)+\lambda \rho w_{x_{2}} \\
& =\rho\left\{f_{11}(\nabla w) v_{x_{1}}+f_{12}(\nabla w) v_{x_{2}}+\lambda w_{x_{2}}\right\} .
\end{aligned}
$$

Since $\left|w_{x_{2}}\right|^{p} \leq \delta^{-(p-2)}\left|w_{x_{2}}\right|^{2}$, it follows from inequalities (4.9), (4.28), and (4.29) that (4.30) holds with $p$ and $K_{1}$ depending on $\delta$.

Part (b) follows from the embedding of $W^{1, p}$ in $C^{\alpha}$ for $p>2$.

For parts (c) and (d) let $N$ denote the $L^{2}$ norm of $w$ on the set $[m-3, m+4] \times I$. Since $\rho$ is bounded below by $\rho_{+}$, it follows from part (b) that

$$
\left|f_{2}(\nabla w)-\lambda w\right|_{C([m, m+1] \times I)} \leq K N
$$

for a $K$ depending on $\delta$. Since $w$ is bounded by $K_{0} N$ from Lemma 4.3, it follows that

$$
\left|f_{2}\left(\nabla w\left(x_{1}, x_{2}\right)\right)\right| \leq K^{\prime} N
$$

on $[m, m+1] \times I$ with $K^{\prime}$ depending on $\delta$. From the same lemma $w_{x_{1}}$ is likewise bounded. Now

$$
\left|f_{2}\left(p_{1}, p_{2}\right)\right|=\left|\frac{2 p_{2}+p_{2}^{2}-p_{1}^{2}}{2\left(1+p_{2}\right)^{2}}\right| \geq \frac{\left|p_{2}\right|}{2\left(1+\delta^{-1}\right)}-\frac{\left|p_{1}\right|}{2 \delta^{3}},
$$

and so the estimate

$$
\left|w_{x_{2}}\right|_{C([m, m+1] \times I)} \leq K_{3} N
$$

follows. 
For part (d) let $p_{i}=w_{x_{i}}\left(x_{1}, x_{2}\right)$ and $\tilde{p}_{i}=w_{x_{i}}\left(\tilde{x}_{1}, \tilde{x}_{2}\right)$ for $i=1,2$, where $\left(x_{1}, x_{2}\right)$ and $\left(\tilde{x}_{1}, \tilde{x}_{2}\right)$ are in $\Omega$. From (4.19) and part (b) the $C^{\alpha}$ norm of $f_{2}(\nabla w)$ on $\Omega$ is bounded by $K_{0} N$, where $K_{0}$ depends on $\delta$. Thus

$$
\left|f_{2}\left(p_{1}, p_{2}\right)-f_{2}\left(p_{1}, \tilde{p}_{2}\right)+f_{2}\left(p_{1}, \tilde{p}_{2}\right)-f_{2}\left(\tilde{p}_{1}, \tilde{p}_{2}\right)\right| \leq K_{0} N \Delta^{\alpha},
$$

where $\Delta$ denotes the distance between $\left(x_{1}, x_{2}\right)$ and $\left(\tilde{x}_{1}, \tilde{x}_{2}\right)$. From Lemma 4.3 the $C^{\alpha}$ norm of $w_{x_{1}}$ on $\Omega$ is bounded by $\tilde{K} N$. Now for $\left(p_{1}, p_{2}\right) \in Q_{\delta}, f_{21}$ is bounded above and $f_{22}$ is bounded below by positive constants depending on $\delta$. If these estimates are used in (4.34), the result is

$$
\left|p_{2}-\tilde{p}_{2}\right| \leq K_{4}^{\prime} N \Delta^{\alpha},
$$

that is, $w_{x_{2}}$ satisfies the desired Hölder condition. This last inequality, combined with part (c) yields the $C^{\alpha}$ bound in (4.33). Q.E.D.

LEMMA 4.5. Let $\Omega$ be any relatively open subset of $T$ on which the density $\rho$ is constant. Then for any solution $(\lambda, w) \in F_{n, \delta, R}, w$ is real analytic on $\Omega$.

PROOF. Extend $\rho$ to be $\rho_{-}$for $x_{2} \leq-h$ and to be $\rho_{+}$for $x_{2} \geq 1-h$. Extend $w$ to be odd about $x_{2}=-h$ for $-2 h<x_{2} \leq-h$ and to be odd about $x_{2}=1-h$ for $1-h \leq x_{2} \leq 2(1-h)$. Then $w$ satisfies (4.17) in each region where $\rho$ is constant. Since $w \in C^{1, \alpha}$ in $\Omega$, the Schauder theory [13, Chapter 6] shows that $w \in C^{2, \alpha}(\Omega)$. Then the results of Morrey $[\mathbf{2 0} ; \mathbf{2 1}$, Chapter 6$]$ show $w$ is analytic. Alternatively a change of variables back to $\psi(x, y)$ which is harmonic where $\rho$ is constant, yields analyticity for $\psi$ and, since $\partial \psi / \partial y>0$, for $y(x, \psi)$ and $w\left(x_{1}, x_{2}\right)$ (cf. (2.34)). Q.E.D.

The estimates up to this point, regarding the restriction of $w$ to $[m, m+1] \times I$, have been independent of $m$. Estimates which reflect the decay in $w$ as $\left|x_{1}\right| \rightarrow \infty$ will also be needed. Before giving these estimates we make the simple observation that since $w_{x_{2}} \geq-1$ for any solution $(\lambda, w) \in F_{n, \delta, R}$ and since $w=0$ for $x_{2}=1-h$, we have

$$
0 \leq w\left(x_{1}, x_{2}\right) \leq 1 \text { in } T .
$$

Recall that we are treating the case $e>0$ (cf. (2.41)) and hence $w>0$ in $T$. In the case $e<0$ one would have $-1 \leq w \leq 0$ in place of (4.35). For $e>0, \partial w / \partial x_{1}<0$ on $(0, \infty) \times I$, and this is used in obtaining the following bound.

LEMMA 4.6. For $(\lambda, w) \in F_{n, \delta, R}$,

$$
\max _{x_{2} \in I} w\left(s, x_{2}\right) \leq R(2 / s)^{1 / 2} .
$$

ProOF. For any $s \in(0, \infty)$, there exists an $\tilde{x}(s) \in(s / 2, s)$ such that

$$
\frac{R^{2}}{2} \geq \int_{s / 2}^{s} \int_{I} w_{x_{2}}^{2}=\frac{s}{2} \int_{I} w_{x_{2}}^{2}\left(\tilde{x}, x_{2}\right) d x_{2},
$$

by the intermediate value theorem. Since $w$ is decreasing for $x_{1}>0$,

$$
\frac{R^{2}}{2} \geq \int_{0}^{\tilde{x}} \int_{I} w^{2} \geq \tilde{x} \int_{I} w^{2}\left(\tilde{x}, x_{2}\right) d x_{2} .
$$

Combining these last two inequalities one has

$$
\max _{x_{2} \in I}\left|w\left(\tilde{x}, x_{2}\right)\right|^{2} \leq 2 \int_{I} w\left(\tilde{x}, x_{2}\right) w_{x_{2}}\left(\tilde{x}, x_{2}\right) d x_{2} \leq \frac{2 R^{2}}{\sqrt{2 \tilde{x} s}} .
$$

Since $2 \tilde{x} \geq s$, inequality (4.36) follows. Q.E.D. 
Now we combine Lemma 4.3, Theorem 4.4, and Lemma 4.6 to obtain the following estimates, wherein $\alpha$ is the Hölder exponent from the earlier results.

Corollary 4.7. Let $d(m)=\min (1, R / \sqrt{m})$. Then for $(\lambda, w) \in F_{n, \delta, R}$

$$
\begin{gathered}
|w|_{C^{0,1}([m, \infty) \times I)} \leq K_{1} d(m), \\
\left|w_{x_{1}}\right|_{C^{\alpha}([m, \infty) \times I)} \leq K_{2} d(m), \\
\left|w_{x_{2}}\right|_{C^{\alpha}(\{[m, \infty) \times I\} \cap \Omega)} \leq K_{3} d(m),
\end{gathered}
$$

and

$$
\left|\rho\left(f_{2}(\nabla w)-\lambda w\right)\right|_{C^{\alpha}([m, \infty) \times I)} \leq K_{4} d(m),
$$

where $\Omega$ is any relatively open subset of $T$ on which $\rho$ is constant and the $K_{i}$, $i=1,2,4$, depend on $\delta$, but are independent of $m, n, R$, and $\Omega$. The constant $K_{3}$ depends only on $\delta$ and $\Omega$.

5. Precompactness of approximating flows. In this section it will be shown that $\bigcup_{n} F_{n, \delta, R}$ is precompact in $\mathbf{R} \times H_{0}^{1}(T)$. The use of Lemma 4.1 will then enable us to obtain certain connected sets of solutions of problem (4.1)-(4.3) as a step toward obtaining the set $S$ in the stronger topology $\mathbf{R} \times\left(H_{0}^{1}(T) \cap C^{0,1}(\bar{T})\right)$ given in Theorem 2.1. Throughout this section $\delta \in(0,1)$ and $R>0$ will be fixed. It will be shown below in Corollary 5.3 that for each fixed $n, F_{n, \delta, R}$ is precompact. The heart of the compactness question concerns a sequence of solutions $\left(\lambda_{n}, w_{n}\right) \in F_{n, \delta, R}$, where $n=1,2,3, \ldots$. The estimates of the previous section allow one to extract a subsequence of these pairs which converges to a pair $(\lambda, w)$, the convergence being weak convergence in $H_{0}^{1}(T)$; norm convergence in $C$, uniformly on bounded sets; and norm convergence in $C^{0,1}$, uniformly on compact subsets of $\bar{T}-\left\{x_{2}=0\right\}$. Moreover, from Lemma 4.2 it can be assumed that $v_{n}=\partial w_{n} / \partial x_{1}$ has $v=\partial w / \partial x_{1}$ as its weak $H_{0}^{1}(T)$ limit. Henceforth it will be assumed that a subsequence has been chosen and renumbered so that $\left(\lambda_{n}, w_{n}\right)$ has the aforementioned properties. Moreover, it can be assumed that $w_{n} \neq 0$ for all $n$; otherwise there would be a subsequence with second components all zero and thus convergent. The notation $I^{ \pm}=\left\{x_{2} \in I \mid \pm x_{2}>0\right\} ; T^{ \pm}=\mathbf{R} \times I^{ \pm}($from $\S 1) ; T_{m}=[m, \infty) \times I ; T_{m}^{ \pm}=$ $[m, \infty) \times I^{ \pm}$; and $d(m)$ from Corollary 4.7 will be used.

THEOREM 5.1. Suppose $\left(\lambda_{n}, w_{n}\right) \in F_{n, \delta, R}$ for $n=1,2, \ldots$, that $\lambda_{n} \rightarrow \lambda$, and that $w_{n} \rightarrow w$ weakly in $H_{0}^{1}(T)$. Then

(a) $\lambda \leq \lambda_{d}$, and

(b) $w_{n} \rightarrow w$ strongly in $H^{1}(B)$ for any bounded $B \subset T$.

(c) The function $w$ is real analytic in $\bar{T}-\left\{x_{2}=0\right\}$, even in $x_{1}$, and satisfies

$$
\begin{gathered}
|w|_{C^{0,1}\left(T_{m}\right)} \leq K d(m), \\
\left|w_{x_{1}}\right|_{C^{\alpha}\left(T_{m}\right)} \leq K d(m) .
\end{gathered}
$$

The function $w_{x_{2}}$ has extensions to $\overline{T^{ \pm}}$, differing on $\left\{x_{2}=0\right\}$ in general, and

$$
\left|w_{x_{2}}\right|_{C^{\alpha}\left(\overline{T_{m}^{ \pm}}\right)} \leq K d(m)
$$


while

$$
\left|\rho_{d}\left\{f_{2}(\nabla w)-\lambda w\right\}\right|_{C^{\alpha}\left(T_{m}\right)} \leq K d(m) .
$$

Here $K$ and $\alpha$ depend on $\delta$, but are independent of $m$ and $R$.

(d) The function $w$ is a solution of (2.36)-(2.39) for the density $\rho_{d}$.

PROOF. (a) From Theorem 2.3 of [4], we have $\lambda_{n} \leq \mu_{n}$. It will be shown in Lemma 5.5 below that $\mu_{n}$ converges to $\lambda_{d}$ as $n \rightarrow \infty$, whence $\lambda \leq \lambda_{d}$.

(b) The convergence of $w_{n}$ in $C^{0,1}$ on compact subsets of $T-\left\{x_{2}=0\right\}$ together with the gradient bounds (4.20) and (4.32) show that $w_{n}$ converges to $w$ in $H^{1}(B)$, yielding part (b). In fact, the Schauder theory provides $C^{2, \alpha}$ estimates on compact subsets of $\bar{T}-\left\{x_{2}=0\right\}$ and thus $w_{n}$ will converge to $w$ in $C^{2}$ on such sets.

(c) The analyticity of $w$ follows as in the proof of Lemma 4.5. Since the estimates in Corollary 4.7 are independent of $n$, the function $w=\lim w_{n}$ inherits properties (4.39)-(4.42) and the estimate on $w_{x_{2}}$ extends to the closures $\overline{T^{ \pm}}$.

(d) Recall that $w_{n}$ converges to $w$ in $C^{2}$ on compact subsets of $T^{ \pm}$so equation (2.36) holds on those sets. The conditions (2.37) and (2.38) are also preserved in the limit. For $\varphi \in C_{0}^{\infty}(T)$ one obtains

$$
\int_{T} \rho_{n} f_{i}\left(\nabla w_{n}\right) \frac{\partial \varphi}{\partial x_{i}}=\lambda_{n} \int_{T} \rho_{n} \frac{\partial\left(w_{n} \varphi\right)}{\partial x_{2}}
$$

from (4.1). Since the vectors grad $w_{n}$ are uniformly bounded and converge to grad $w$ on $T^{ \pm}$, it follows that

$$
\begin{aligned}
\int_{T} \rho_{d} f_{i}(\nabla w) \frac{\partial \varphi}{\partial x_{i}} & =\lambda \int_{T^{-}} \rho_{d} \frac{\partial}{\partial x_{2}}(w \varphi)+\lambda \int_{T^{+}} \rho_{d} \frac{\partial}{\partial x_{2}} w \varphi \\
& =\lambda\left(\rho_{-}-\rho_{+}\right) \int_{-\infty}^{\infty} w\left(x_{1}, 0\right) \varphi\left(x_{1}, 0\right) d x_{1}
\end{aligned}
$$

showing that $w$ satisfies (2.39). Q.E.D.

LEMMA 5.2. Suppose $(\lambda, w) \in F_{n, \delta, R}$ and that $\theta=1-\left(\lambda / \mu_{n}\right)>0$. Then for $m>0$

$$
|w|_{H^{1}\left(T_{m}\right)} \leq K / \sqrt{m}
$$

where $K$ depends on $\delta, R$, and $\theta$.

PROOF. Since $|w|_{H_{0}^{1}(T)} \leq R$ by assumption, the inequality (5.6) need be demonstrated only for $m$ larger than some $m_{0}$. If equation (4.1) is multiplied by $w$ and integrated over $T_{m}$, it yields

$$
\begin{aligned}
\frac{1}{2} \int_{T_{m}} \rho_{n} \frac{2+w_{x_{2}}}{\left(1+w_{x_{2}}\right)^{2}}|\nabla w|^{2} & =-\int_{I} \rho_{n} w\left(m, x_{2}\right) f_{1}(\nabla w) d x_{2}-\lambda \int_{T_{m}} \rho_{n}^{\prime} w^{2} \\
& \leq-\int_{I} \rho_{n} w\left(m, x_{2}\right) f_{1}(\nabla w) d x_{2}+\frac{\lambda}{\mu_{n}} \int_{T_{m}} \rho_{n}|\nabla w|^{2}
\end{aligned}
$$

from the characterization (3.2) of $\mu_{n}$. For $m \geq m_{0}, m_{0}$ depending on $\delta$ and $R$,

$$
\left(2+w_{x_{2}}\right) / 2\left(1+w_{x_{2}}\right)^{2}>1-(\theta / 2) \text { and } \quad-w f_{1}(\nabla w) \leq 2 w w_{x_{1}} \quad \text { for } x_{1} \geq m \text {. }
$$

Hence, from (5.7)

$$
\frac{1}{2} \theta \rho_{+} \int_{T_{m}}|\nabla w|^{2} \leq \rho_{-} \int_{I}\left|w\left(m, x_{2}\right)\right|\left|w_{x_{1}}\left(m, x_{2}\right) d x_{2}\right|
$$

which, with Corollary 4.7 and the Poincaré inequality, provides (5.6). Q.E.D. 
COROllaRY 5.3. For each fixed $n, F_{n, \delta, R}$ is precompact in $\mathbf{R} \times H_{0}^{1}(T)$.

ProOF. Let $\left(\lambda_{k}, w_{k}\right), k=1,2,3, \ldots$, be a sequence in $F_{n, \delta, R}$. If a subsequence converges to $\left(\mu_{n}, 0\right)$, we are done. If not, then $\left|\lambda_{k}-\mu_{n}\right|+\left|w_{k}\right|_{H^{1}(T)} \geq \eta>0$ for all large $k$. Suppose a subsequence, still denoted $\left(\lambda_{k}, w_{k}\right)$, is such that $\lambda_{k}$ converges to $\mu_{n}$ as $k \rightarrow \infty$. Since $w_{k}$ lies in the ball of radius $R$ in $H^{1}(T), u_{k}$ lies in the ball of radius $K_{2} R$ in $H^{1}(S)$ by (3.8). According to $[\mathbf{4}, \S 5]$, the collection $\left\{\left(\lambda_{k}, u_{k}\right)\right\}$, $k=1,2, \ldots$, is precompact in $\mathbf{R} \times\left(L^{2}(S) \cap C(\bar{S})\right)$. (The exponent $\sigma$ in (1.4) of [4] is 1 as established in the discussion following (7.14) of that paper.) It follows that a subsequence, still denoted $\left(\lambda_{k}, u_{k}\right)$, converges to $\left(\mu_{n}, u\right)$ in $\mathbf{R} \times\left(L^{2}(S) \cap C(\bar{S})\right)$. From local elliptic estimates one sees that $\left(\mu_{n}, u\right)$ is a solution and that the $u_{k}$ converge to $u$ in $C^{2}(\bar{S})$. Then Theorems 2.3 and 2.4 of $[4]$ show that $u=0$. From this information one can conclude that the $y$ derivatives of the corresponding $\psi_{k}$ are bounded below by $K>0$, uniformly on $S$ and uniformly in $m$ for $m$ large. Elliptic estimates, used once more, show that the $L^{2}$ convergence of $u_{k}$ to zero implies convergence to zero in $H^{1}(S)$, and (3.7) implies convergence of $w_{k}$ to zero in $H^{1}(T)$. However, from an earlier inequality it follows that $\left|w_{k}\right|_{H^{1}(T)} \geq \eta / 2>0$ for all large $k$, a contradiction.

From the last paragraph it is clear that $\lambda_{k} / \mu_{n} \leq \theta<1$ for all $k$, and thus (5.6) holds for all $w_{k}$, uniformly in $k$. The precompactness of $\left\{w_{k}\right\}, k=1,2, \ldots$, in $H^{1}(B)$ for each bounded set $B \subset T$ follows as in the proof of Theorem 5.1. These two properties combine to yield precompactness in $\mathbf{R} \times H_{0}^{1}(T)$. Q.E.D.

To show precompactness of $\bigcup_{n} F_{n, \delta, R}$ it will now suffice to show that the sequence $\left\{\left(\lambda_{n}, w_{n}\right)\right\}, n=1,2, \ldots$, selected at the outset of this section, converges in $\mathbf{R} \times$ $H_{0}^{1}(T)$. We next show this together with some of the properties of the branch required for Theorem 2.1. For the case $\lim \lambda_{n}=\lambda<\lambda_{d}$, we need merely quote results already established to see the convergence of $w_{n}$. For $\lambda=\lambda_{d}$, a delicate analysis must be carried out using the nonlinearity in equation (4.1).

The case $\lambda<\lambda_{d}$.

THEOREM 5.4. Suppose $\left(\lambda_{n}, w_{n}\right) \in F_{n, \delta, R}$ satisfy $\lambda_{n} \rightarrow \lambda<\lambda_{d}$ and $w_{n} \rightarrow w$ weakly in $H_{0}^{1}(T)$ as $n \rightarrow \infty$. Then

(a) $w_{n}$ converges to $w$ strongly in $H_{0}^{1}(T)$.

(b) $w>0$ in $T$ and $\partial w / \partial x_{1}<0$ for $x_{1}>0$ and $x_{2} \in I$.

PROOF. (a) It will be shown in Lemma 5.5(c) that $\mu_{n}$ approaches $\lambda_{d}$ as $n \rightarrow \infty$. Hence inequality (5.6) holds for $w_{n}$, uniformly in $n$ for all large $n$. This decay in the tail together with Theorem 5.1(b) yields strong convergence in $H_{0}^{1}(T)$.

(b) From (5.5) it follows that

$$
\int_{T} \rho_{d} \tilde{f}_{i j} \frac{\partial w}{\partial x_{i}} \frac{\partial \varphi}{\partial x_{j}} \geq 0
$$

for all nonnegative $\varphi \in C_{0}^{\infty}(T)$, where $\tilde{f}_{i j}$ is defined by (4.23). Hence, from the strong maximum principle [13, Theorem 8.19], either $w \equiv 0$ or $w>0$ in $T$. The function $v=\partial w / \partial x_{1}$ is in $H_{0}^{1}(T)$ by Lemma 4.2, and is the weak $H^{1}$ limit of $v_{n}=\partial w_{n} / \partial x_{1}$. The equation (4.12) is satisfied by $\left(\lambda_{n}, w_{n}, v_{n}\right)$, and since $v_{n}<0$ for $x_{1}>0$, one obtains

$$
\int_{T} \rho_{d} f_{i j}(\nabla w) \frac{\partial v}{\partial x_{i}} \frac{\partial \varphi}{\partial x_{j}} \leq 0
$$


for all nonnegative $\varphi \in C_{0}^{\infty}([0, \infty) \times I)$. From the strong maximum principle $v \equiv 0$ or $v<0$ on $(0, \infty) \times I$, corresponding to the case $w \equiv 0$ or $w>0$, respectively. If $w \equiv 0$, then the functions $w_{n}$, which are even in $x_{1}$ and decreasing in $x_{1}$ on $(0, \infty) \times I$, must converge uniformly to zero on $T$. The gradient estimates $(4.20)$ and (4.32) show that $\nabla w_{n}$ converges uniformly to zero on $T$, and thus $w_{n}$ converges to zero in $C^{0,1}(T)$. The analogue of (5.7) taken over all of $T$ yields

$$
\int_{T} \rho_{n} \frac{2+\left(w_{n}\right)_{x_{2}}}{2\left(1+\left(w_{n}\right)_{x_{2}}\right)^{2}}\left|\nabla w_{n}\right|^{2} \leq \frac{\lambda_{n}}{\mu_{n}} \int_{T} \rho_{n}\left|\nabla w_{n}\right|^{2} .
$$

Since $\lambda_{n} / \mu_{n}$ converges to $\lambda / \lambda_{d}<1$ and $\left|\nabla w_{n}\right| \rightarrow 0$ uniformly on $T$ as $n \rightarrow \infty$, (5.8) leads to a contradiction for large $n$. Thus $w>0$ on $T$ and $\partial w / \partial x_{1}<0$ for $x_{1}>0$. Q.E.D.

The case $\lambda=\lambda_{d}$. As stated earlier, it is assumed that we are working with a sequence $\left(\lambda_{n}, w_{n}\right) \in F_{n, \delta, R}$ for which $\lambda_{n} \rightarrow \lambda=\lambda_{d}$ and $w_{n}$ converges to $w$ in the weak topology of $H_{0}^{1}(T)$ as $n \rightarrow \infty$. In addition, the conclusion of Theorem 5.1 hold. The plan here is to show in Theorem 5.6 that the weak limit is $w \equiv 0$ and then to show in Theorem 5.7 that $w_{n} \rightarrow 0$ strongly in $H_{0}^{1}(T)$ so that the limiting point is the bifurcation point $\left(\lambda_{d}, 0\right)$.

To begin recall that $\mu_{n}$ is defined by

$$
\frac{1}{\mu_{n}}=\max _{u \in H_{0}^{1}(I)} \frac{-\int_{I} \rho_{n}^{\prime} u^{2}}{\int_{I} \rho_{n}\left(u^{\prime}\right)^{2}} .
$$

Let $\theta_{n}=\theta_{n}\left(x_{2}\right)$ be the associated positive maximizer normalized by $\int_{I} \rho_{n} \theta_{n}^{2}=1$. The function $\theta_{n}$ satisfies the Euler equation

$$
\left(\rho_{n} \theta_{n}^{\prime}\right)^{\prime}=\mu_{n} \rho_{n}^{\prime} \theta_{n}
$$

An associated quantity $\alpha_{n}$ is defined by

$$
\alpha_{n}(\lambda)=\max _{u \in H_{0}^{1}(I)}\left\{\frac{-\lambda \int_{I} \rho_{n}^{\prime} u^{2}-\int_{I} \rho_{n}\left(u^{\prime}\right)^{2}}{\int_{I} \rho_{n} u^{2}}\right\}
$$

for each $\lambda \in \mathbf{R}$. Let $\gamma_{n}=\gamma_{n}\left(x_{2}\right)$ be the positive maximizer in (5.11) when $\lambda=\lambda_{n}$; again suppose that $\int_{I} \rho_{n} \gamma_{n}^{2}=1$. The function $\gamma_{n}$ satisfies the Euler equation

$$
\left(\rho_{n} \gamma_{n}^{\prime}\right)^{\prime}-\lambda_{n} \rho_{n}^{\prime} \gamma_{n}=\alpha_{n}\left(\lambda_{n}\right) \rho_{n} \gamma_{n}
$$

Of course, $\theta_{n}$ and $\gamma_{n}$ both vanish at the endpoints of $I=(-h, 1-h)$.

LEMMA 5.5. Let $\left(\lambda_{n}, w_{n}\right)$ be in $F_{n, \delta, R} \backslash\left\{\left(\mu_{n}, 0\right)\right\}$. Then

(a) $\alpha_{n}\left(\lambda_{n}\right)<0$.

(b) Assume $\lambda_{n} \rightarrow \lambda_{d}$ as $n \rightarrow \infty$. Then the functions $\left\{\theta_{n}\right\}$ and $\left\{\gamma_{n}\right\}, n=1,2, \ldots$, are bounded in $C^{1}(\bar{I})$ uniformly in $n$ and for each $\varepsilon>0$ converge in $C(\bar{I}) \cap C^{1}\left(\bar{I}_{\varepsilon}\right)$, $I_{\varepsilon}=I \cap\left\{\left|x_{2}\right|>\varepsilon\right\}$, to the function

$$
\gamma_{d}\left(x_{2}\right)= \begin{cases}\sigma\left(x_{2}+h\right), & x_{2} \leq 0, \\ \frac{\sigma h}{h-1}\left(x_{2}-1+h\right), & x_{2} \geq 0,\end{cases}
$$

where $\sigma^{2}=3 / h^{2}\left(\rho_{-} h+\rho_{+}(1-h)\right)$. Let

$$
Q(u)=\frac{-\int_{I} \rho_{d}^{\prime} u^{2}}{\int_{I} \rho_{d}\left(u^{\prime}\right)^{2}}=\frac{\left(\rho_{-}-\rho_{+}\right) u^{2}(0)}{\int_{I} \rho_{d}\left(u^{\prime}\right)^{2}} .
$$


Then $\gamma_{d}$ is the positive maximizer of $Q(u)$ for $u \in H_{0}^{1}(I)$ and

$$
Q\left(\gamma_{d}\right)=1 / \lambda_{d}
$$

where $\lambda_{d}$ is defined by (2.40).

(c) $\lim _{n \rightarrow \infty} \mu_{n}=\lambda_{d}$.

PROOF. (a) From the characterization (5.11) $\alpha_{n}$ is a strictly increasing function of $\lambda$ and from (5.9), $\alpha_{n}\left(\mu_{n}\right)=0$. As noted in the proof of Corollary 5.3, for $\left(\lambda_{n}, w_{n}\right) \in F_{n, \delta, R} \backslash\left\{\left(\mu_{n}, 0\right)\right\}, \lambda_{n}<\mu_{n}$, and so $\alpha_{n}\left(\lambda_{n}\right)<0$.

(b)-(c) Since evaluation at $x_{2}=0$ is a compact mapping for functions in $H_{0}^{1}(I)$, the maximum of $Q(u)$ is attained, and the form of $Q$ ensures that this maximizer must be linear on $I^{+}$and $I^{-}$. A calculation shows that $\gamma_{d}$ is the positive maximizer which has the normalization $\int_{I} \rho_{d} \gamma_{d}^{2}=1$ and that $Q\left(\gamma_{d}\right)=\lambda_{d}^{-1}$.

Suppose the maximizer $\theta_{n}$ for (5.9) is renormalized to have $\max \theta_{n}=1$ on $I$ and that its maximum occurs at $\tilde{x}_{2}$. Since $\theta_{n}^{\prime}\left(\tilde{x}_{2}\right)=0$, an integration of (5.10) from $\tilde{x}_{2}$ to $x_{2}$ yields

$$
\rho_{n} \theta_{n}^{\prime}\left(x_{2}\right)=\int_{\tilde{x}_{2}}^{x_{2}} \rho_{n}^{\prime} \theta_{n}
$$

and so $\left|\theta_{n}^{\prime}\left(x_{2}\right)\right| \leq\left(\rho_{-}-\rho_{+}\right) / \rho_{+}$. This bound easily implies that the eigenfunction normalized by $\int_{I} \rho_{n} \theta_{n}^{2}=1$ must also satisfy $\left|\theta_{n}^{\prime}\right| \leq K_{1}$, where $K_{1}$ depends only on $\rho_{ \pm}$and $h$. On $I_{\varepsilon}$, that is, where $\left|x_{2}\right|>\varepsilon$, the derivative $\rho_{n}^{\prime}=0$ for $n>1 / \varepsilon$ and hence $\theta_{n}^{\prime \prime}=0$. It follows from the Arzela-Ascoli theorem that a subsequence of $\left\{\theta_{n}\right\}$ converges as asserted to a function $\theta_{\infty}$ which must be linear on $I^{+}$and $I^{-}$. Since $\int_{I} \rho_{d} \theta_{\infty}^{2}=1$, a calculation shows that $\theta_{\infty}$ must be $\gamma_{d}$ and the uniqueness of the limit shows that the whole sequence converges. The maximum value in (5.9) converges to

$$
\frac{\left(\rho_{-}-\rho_{+}\right) \gamma_{d}^{2}(0)}{\int_{I} \rho_{d}\left(\gamma_{d}^{\prime}\right)^{2}}
$$

and thus $\mu_{n} \rightarrow \lambda_{d}$ as $n \rightarrow \infty$.

All values of $\lambda$ under consideration for a fixed $n$ lie in the interval $\left[0, \mu_{n}\right]$, and since $\alpha_{n}$ is increasing, $\alpha_{n}(0) \leq \alpha_{n}(\lambda) \leq \alpha_{n}\left(\mu_{n}\right)=0$. Moreover, the quantity $\alpha_{n}(0)$ is readily seen to be bounded below by a negative constant $K_{2}$ depending on $\rho_{ \pm}$ and $h$, but not on $n$. By integrating the equation (5.12) for $\gamma_{n}$ as was done with (5.10), one finds that $\left|\gamma_{n}^{\prime}\right| \leq K_{3}$ on $I$ and $\left|\gamma_{n}^{\prime \prime}\right| \leq K_{4}\left|\alpha_{n}\left(\lambda_{n}\right)\right|$ on $I_{\varepsilon}$ for $n>1 / \varepsilon$. To see that $\gamma_{n}$, like $\theta_{n}$, converges to $\gamma_{d}$, it suffices to show that $\left|\alpha_{n}\left(\lambda_{n}\right)\right| \rightarrow 0$ as $n \rightarrow \infty$. Since $\lambda_{n} \rightarrow \lambda_{d}, \mu_{n} \rightarrow \lambda_{d}$, and $\alpha_{n}\left(\mu_{n}\right)=0$, the desired convergence will follow if it is shown that $\alpha_{n}$ has a modulus of continuity independent of $n$. To see this let $u_{r}$ be a maximizer associated with $\alpha_{n}(r)$ in (5.11) and suppose $\int_{I} \rho_{n} u_{r}^{2}=1$. Then for $r>s$,

$$
\begin{aligned}
\alpha_{n}(r) & =-r \int_{I} \rho_{n}^{\prime} u_{r}^{2}-\int_{I} \rho_{n}\left(u_{r}^{\prime}\right)^{2} \\
& =-(r-s) \int_{I} \rho_{n}^{\prime} u_{r}^{2}-s \int_{I} \rho_{n}^{\prime} u_{r}^{2}-\int_{I} \rho_{n}\left(u_{r}^{\prime}\right)^{2} \\
& \leq(r-s) K_{5}\left(\rho_{-}-\rho_{+}\right)+\alpha_{n}(s),
\end{aligned}
$$

since $u_{r}$ is an admissible function in the definition of $\alpha_{n}(s)$. Since $\alpha_{n}$ is an increasing function, it follows from (5.15) that

$$
\left|\alpha_{n}(r)-\alpha_{n}(s)\right| \leq K_{6}|r-s|
$$

with $K_{6}$ independent of $n$. Q.E.D. 
THEOREM 5.6. Let $\delta \in(0,1)$ and $R>0$ be fixed, and suppose $\left(\lambda_{n}, w_{n}\right) \in$ $F_{n, \delta, R}$ for $n=1,2, \ldots$. If $\lambda_{n} \rightarrow \lambda_{d}$ and $w_{n}$ converges weakly to $w \in H_{0}^{1}(T)$ as $n \rightarrow \infty$, then

(a) $w \equiv 0$ in $T$.

(b) $\left|w_{n}\right|$ and $\left|\nabla w_{n}\right|$ converge to zero uniformly in $\bar{T}$ as $n \rightarrow \infty$.

Proof. (a) The argument revolves about the behavior of $w_{n}$ for large $x_{1}$ and that behavior, as we shall see, is reflected in the projection of $w_{n}$ along the eigenfunction $\gamma_{n}$ of (5.12). Let

$$
w_{n}\left(x_{1}, x_{2}\right)=F_{n}\left(x_{1}\right) \gamma_{n}\left(x_{2}\right)+R_{n}\left(x_{1}, x_{2}\right),
$$

where

$$
F_{n}\left(x_{1}\right)=\int_{I} \rho_{n}\left(x_{2}\right) w_{n}\left(x_{1}, x_{2}\right) \gamma_{n}\left(x_{2}\right)
$$

with $\gamma_{n}$ normalized by

$$
\int_{I} \rho_{n} \gamma_{n}^{2}=1
$$

It follows that $\gamma_{n}$ and $R_{n}$ are orthogonal with respect to $\rho_{n}$ for each fixed $x_{1}$ :

$$
\int_{I} \rho_{n} R_{n} \gamma_{n} d x_{2}=0, \quad x_{1} \in \mathbf{R} \text {. }
$$

Let

$$
G_{n}\left(x_{1}\right)=\int_{I} \gamma_{n} \rho_{n} \frac{\left(w_{n}\right)_{x_{1}}}{1+\left(w_{n}\right)_{x_{2}}} .
$$

If the principal equation (4.1) is multiplied by $\gamma_{n}$ and the result integrated over $I$, there emerges

$$
\frac{d}{d x_{1}} G_{n}=\lambda_{n} \int_{I} \gamma_{n} \rho_{n}^{\prime} w_{n}+\int_{I} \gamma_{n}^{\prime} \rho_{n} f_{2}\left(\nabla w_{n}\right)
$$

after an integration by parts in the integral containing $f_{2}$. A simple calculation shows that

$$
f_{2}\left(p_{1}, p_{2}\right)=p_{2}-\frac{3}{2} p_{2}^{2}+A\left(p_{1}, p_{2}\right)
$$

where

$$
A=\left(3 p_{2}^{4}+4 p_{2}^{3}-p_{1}^{2}\right) / 2\left(1+p_{2}\right)^{2} .
$$

From equation (5.12) for $\gamma_{n}$ one has

$$
\int_{I} \gamma_{n}^{\prime} \rho_{n}\left(w_{n}\right)_{x_{2}}=-\int_{I} w_{n}\left(\rho_{n} \gamma_{n}^{\prime}\right)^{\prime}=-\int_{I} \alpha_{n}\left(\lambda_{n}\right) w_{n} \rho_{n} \gamma_{n}-\lambda_{n} \int_{I} w_{n} \rho_{n}^{\prime} \gamma_{n},
$$

and the use of this last identity with the expression (5.22) in (5.21) yields

$$
G_{n}^{\prime}=-\alpha_{n}\left(\lambda_{n}\right) \int_{I} \rho_{n} w_{n} \gamma_{n}-\frac{3}{2} \int_{I} \gamma_{n}^{\prime} \rho_{n}\left[\left(w_{n}\right)_{x_{2}}\right]^{2}+\int_{I} \gamma_{n}^{\prime} \rho_{n} A\left(\nabla w_{n}\right) .
$$


With the use of (5.16) the second integral in (5.24) can be written

$$
\begin{aligned}
& \frac{3}{2} \int_{I} \gamma_{n}^{\prime} \rho_{n}\left(F_{n} \gamma_{n}^{\prime}+\left(R_{n x_{2}}\right)^{2}\right. \\
& \quad=\frac{3}{2} F_{n}^{2} \int_{I} \rho_{n}\left(\gamma_{n}^{\prime}\right)^{3}+3 F_{n} \int_{I} \rho_{n}\left(\gamma_{n}^{\prime}\right)^{2}\left(R_{n}\right)_{x_{2}}+\frac{3}{2} \int_{I} \gamma_{n}^{\prime} \rho_{n}\left(R_{n}\right)_{x_{2}}^{2},
\end{aligned}
$$

where here and in what follows the convention $\left(R_{n}\right)_{x_{2}}^{2}$ is used for $\left[\left(R_{n}\right)_{x_{2}}\right]^{2}$. Now use the definition of $\gamma_{d}$ in (5.13) to compute

$$
\begin{aligned}
\tilde{e} & =\lim _{n \rightarrow \infty} \frac{3}{2} \int_{I} \rho_{n}\left(\gamma_{n}^{\prime}\right)^{3}=\frac{3}{2} \int_{I} \rho_{d}\left(\gamma_{d}^{\prime}\right)^{3} \\
& =\frac{3}{2} \frac{3^{3 / 2}}{\left[\rho_{-} h+\rho_{+}(1-h)\right]^{3 / 2}}\left(\frac{\rho_{-}}{h^{2}}-\frac{\rho_{+}}{(1-h)^{2}}\right) .
\end{aligned}
$$

Since the expression $\left(\rho_{-} / h^{2}\right)-\left(\rho_{+} /(1-h)^{2}\right)=e$ (cf. $\left.\S 2.2\right)$ is positive by assumption, $\tilde{e}>0$. Only the positivity of $\tilde{e}$ is important in what follows, so to simplify notation we omit the tilde. We conclude that, for any $\varepsilon>0$ and all $n>N(\varepsilon)$,

$$
\left|\frac{3}{2} F_{n}^{2} \int_{I} \rho_{n}\left(\gamma_{n}^{\prime}\right)^{3}-e F_{n}^{2}\right| \leq \varepsilon F_{n}^{2}, \quad x_{1} \in \mathbf{R} .
$$

The use of this last inequality in (5.25) together with Young's inequality gives

$$
\left|\frac{3}{2} \int_{I} \gamma_{n}^{\prime} \rho_{n}\left(w_{n}\right)_{x_{2}}^{2}-e F_{n}^{2}\right| \leq 2 \varepsilon F_{n}^{2}+K_{1} \int_{I} \rho_{n}\left(R_{n}\right)_{x_{2}}^{2}
$$

where $K_{1}$ depends on $\varepsilon$, but is independent of $n$ and $x_{1}$. From the form of $A$ given in (5.23) and from the decay of $w_{n}$ and $\nabla w_{n}$ as $x_{1} \rightarrow \infty$ given by (4.39), it follows that

$$
\left|A\left(\nabla w_{n}\right)\right| \leq K_{2}\left(\left(F_{n}^{\prime}\right)^{2}+F_{n}^{3}+\left|\nabla R_{n}\right|^{2}\right)
$$

for $x_{1}$ larger than some value $X$. The use of the last two estimtes in (5.24) yields

$$
\left|G_{n}^{\prime}+\alpha_{n}\left(\lambda_{n}\right) F_{n}+e F_{n}^{2}\right| \leq 2 \varepsilon F_{n}^{2}+K(\varepsilon) \int_{I} \rho_{n}\left\{\left(F_{n}^{\prime}\right)^{2}+F_{n}^{3}+|\nabla R|^{2}\right\}
$$

for $n \geq N(\varepsilon)$ and $x_{1} \geq X$ where $K(\varepsilon)$ is independent of $n$ and $x_{1}$.

Note that the term $G_{n}$ approximates $F_{n}^{\prime}\left(x_{1}\right)$ for $w_{x_{2}}$ small and thus (5.29) is akin to a differential inequality for $F_{n}$. The aim is to show that in the limit as $n \rightarrow \infty$ and $\alpha_{n}\left(\lambda_{n}\right) \rightarrow 0,(5.29)$ is inconsistent with having a positive function $w\left(x_{1}, x_{2}\right)$ which decreases in $x_{1}$ for $x_{1}>0$. To arrive at this contradiction we first estimate the integral in (5.29).

If the principal equation (4.1) is multiplied by $w_{n}$ and the two sides are integrated over $I$ there results

$$
\begin{aligned}
\int_{I} \rho_{n}\left(w_{n}\right)_{x_{2}} f_{2}\left(\nabla w_{n}\right)= & -\lambda_{n} \int_{I} \rho_{n}^{\prime} w_{n}^{2}+\int_{I} \rho_{n} w_{n} \frac{\partial}{\partial x_{1}} f_{1}\left(\nabla w_{n}\right) \\
= & -\lambda_{n} \int_{I} \rho_{n}^{\prime} w_{n}^{2}+\frac{d}{d x_{1}} \int_{I} \rho_{n} w_{n} f_{1}\left(\nabla w_{n}\right) \\
& -\int_{I} \rho_{n}\left(w_{n}\right)_{x_{1}} f_{1}\left(\nabla w_{n}\right)
\end{aligned}
$$


Let

$$
U_{n}\left(x_{1}\right)=\int_{I} \rho_{n} w_{n} f_{1}\left(\nabla w_{n}\right)
$$

Then since $f_{2}\left(p_{1}, p_{2}\right)=p_{2}-\left(2 p_{2}^{3}+3 p_{2}^{2}+p_{1}^{2}\right) / 2\left(1+p_{2}\right)^{2}$, equation (5.30) yields

$$
\begin{aligned}
\int_{I} \rho_{n}\left(w_{n}\right)_{x_{2}}^{2}+\int_{I} \rho_{n} \frac{\left(w_{n}\right)_{x_{1}}^{2}}{1+\left(w_{n}\right)_{x_{2}}} \\
=-\lambda_{n} \int_{I} \rho_{n}^{\prime} w_{n}^{2}+\frac{d}{d x_{1}} U_{n}\left(x_{1}\right) \\
\quad+\frac{1}{2} \int_{I} \rho_{n} \frac{\left(w_{n}\right)_{x_{2}}}{\left(1+\left(w_{n}\right)_{x_{2}}\right)^{2}}\left\{2\left(w_{n}\right)_{x_{2}}^{3}+3\left(w_{n}\right)_{x_{2}}^{2}+\left(w_{n}\right)_{x_{1}}^{2}\right\}
\end{aligned}
$$

Now from the representation $w_{n}=F_{n} \gamma_{n}+R_{n}$, the equation (5.12) for $\gamma_{n}$, and the orthogonality (5.19)

$$
\int_{I} \rho_{n}\left(w_{n}\right)_{x_{2}}^{2}=F_{n}^{2} \int_{I} \rho_{n}\left(\gamma_{n}^{\prime}\right)^{2}-2 \lambda_{n} F_{n} \int_{I} \rho_{n}^{\prime} \gamma_{n} R_{n}+\int_{I} \rho_{n}\left(R_{n}\right)_{x_{2}}^{2} .
$$

A. second use of the representation for $w_{n}$ yields

$$
\int_{I} \rho_{n}\left(w_{n}\right)_{x_{2}}^{2}+\lambda_{n} \int_{I} \rho_{n}^{\prime} w_{n}^{2}=-\alpha_{n}\left(\lambda_{n}\right) F_{n}^{2}+\lambda_{n} \int_{I} \rho_{n}^{\prime} R_{n}^{2}+\int_{I} \rho_{n}\left(R_{n}\right)_{x_{2}}^{2}
$$

Now define $\tau_{n}$ by

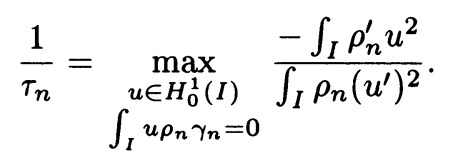

Since an extra condition on $u$ has been added to the characterization of $\mu_{n}$ in (5.9), it is natural that $\tau_{n}>\mu_{n}$ and this is shown in [4, Lemma 3.1]. An argument similar to that given in Lemma 5.5 shows that as $n \rightarrow \infty$ the numbers $\tau_{n}$ approaches a limit $\tau_{\infty}>\lambda_{d}$ so that $\lambda_{n} / \tau_{n} \rightarrow \lambda_{d} / \tau_{\infty}<1$. Thus $1-\lambda_{n} / \tau_{n} \geq \theta>0$ for all large $n$. Since

$$
\int_{I} R_{n}\left(x_{1}, x_{2}\right) \rho_{n}\left(x_{2}\right) \gamma_{n}\left(x_{2}\right) d x_{2}=0
$$

for each $x_{1}$,

$$
-\lambda_{n} \int_{I} \rho_{n}^{\prime} R_{n}^{2} \leq \frac{\lambda_{n}}{\tau_{n}} \int_{I} \rho_{n}\left(R_{n}\right)_{x_{2}}^{2} \leq(1-\theta) \int_{I} \rho_{n}\left(R_{n}\right)_{x_{2}}^{2}
$$

The use of this last inequality in (5.34) gives

$$
\int_{I} \rho_{n}\left(w_{n}\right)_{x_{2}}^{2}+\lambda_{n} \int_{I} \rho_{n}^{\prime} w_{n}^{2} \geq-\alpha_{n}\left(\lambda_{n}\right) F_{n}^{2}+\theta \int_{I} \rho_{n}\left(R_{n}\right)_{x_{2}}^{2}
$$

for all large $n$. The inequality (5.36) will be used in (5.32). First, however, note that because of the decay given by Corollary 4.7, for any $\varepsilon>0$ there is an $X(\varepsilon)$, independent of $n$, such that

$$
\int_{I} \rho_{n} \frac{\left(w_{n}\right)_{x_{1}}^{2}}{1+\left(w_{n}\right)_{x_{2}}} \geq(1-\varepsilon) \int_{I} \rho_{n}\left(w_{n}\right)_{x_{1}}^{2}
$$


provided $x_{1}>X(\varepsilon)$. This same decay coupled with the decomposition (5.16) of $w_{n}$ and Young's inequality gives

$$
\begin{gathered}
\frac{1}{2} \int_{I} \rho_{n} \frac{\left(w_{n}\right)_{x_{2}}}{\left(1+\left(w_{n}\right)_{x_{2}}\right)^{2}}\left\{2\left(w_{n}\right)_{x_{2}}^{3}+3\left(w_{n}\right)_{x_{2}}^{2}+\left(w_{n}\right)_{x_{1}}^{2}\right\} \\
\leq K_{1} F_{n}^{3}+K_{2} \varepsilon \int_{I} \rho_{n}\left(R_{n}\right)_{x_{2}}^{2}+\varepsilon \int_{I} \rho_{n}\left(w_{n}\right)_{x_{1}}^{2}
\end{gathered}
$$

for $x_{1}>X(\varepsilon)$ (taking $X(\varepsilon)$ larger if necessary). The last three inequalities, with $\varepsilon$ chosen so that $K_{2} \varepsilon<\theta / 2$, combined with equation (5.32), produce the inequality

$$
\frac{\theta}{2} \int_{I} \rho_{n}\left(R_{n}\right)_{x_{2}}^{2}-\alpha_{n}\left(\lambda_{n}\right) F_{n}^{2}+(1-2 \varepsilon) \int_{I} \rho_{n}\left(w_{n}\right)_{x_{1}}^{2} \leq U_{n}^{\prime}\left(x_{1}\right)+K_{1} F_{n}^{3}
$$

valid for $n>N(\varepsilon)$ and $x_{1}>X(\varepsilon)$. To further decompose terms using $F_{n}$ and $R_{n}$, note that

$$
\begin{aligned}
\int_{I} \rho_{n}\left(w_{n}\right)_{x_{1}}^{2} & =\left(F_{n}^{\prime}\right)^{2} \int_{I} \rho_{n} \gamma_{n}^{2}+2 F_{n}^{\prime} \int_{I} \rho_{n} \gamma_{n}\left(R_{n}\right)_{x_{1}}+\int_{I} \rho_{n}\left(R_{n}\right)_{x_{1}}^{2} \\
& =\left(F_{n}^{\prime}\right)^{2}+\int_{I} \rho_{n}\left(R_{n}\right)_{x_{1}}^{2}
\end{aligned}
$$

using (5.18) and (5.19). It can be assumed that $\varepsilon$ is chosen so that $\theta / 2<1-2 \varepsilon$. Then since $\alpha_{n}\left(\lambda_{n}\right)<0$, it follows from (5.39) and (5.40) that

$$
\left(F_{n}^{\prime}\right)^{2}+\int_{I} \rho_{n}\left|\nabla R_{n}\right|^{2} \leq \frac{2}{\theta} U_{n}^{\prime}+K^{\prime} F_{n}^{3}
$$

For these same parameter ranges, the use of (5.41) in (5.29) yields

$$
\left|G_{n}^{\prime}+\alpha_{n}\left(\lambda_{n}\right) F_{n}+e F_{n}^{2}\right| \leq 2 \varepsilon F_{n}^{2}+K U_{n}^{\prime}+K F_{n}^{3}
$$

where $e$ is positive and $K$ depends on $\varepsilon$, but not on $n$ or $x_{1}$. Since $F_{n}\left(x_{1}\right) \rightarrow 0$ as $x_{1} \rightarrow \infty$, uniformly in $n$, we may assume that $X(\varepsilon)$ is chosen so that $K F_{n}\left(x_{1}\right)<\varepsilon$ for $x_{1}>X(\varepsilon)$, leading to

$$
\left|G_{n}^{\prime}+\alpha_{n}\left(\lambda_{n}\right) F_{n}+e F_{n}^{2}\right| \leq 3 \varepsilon F_{n}^{2}+K U_{n}^{\prime} .
$$

Now one can integrate the inequality (5.43) (without the absolute value taken on the left side) over the interval $\left[x_{1}, \hat{x}_{1}\right]$ where $X(\varepsilon)<x_{1}<\hat{x}_{1}<\infty$. Next let $n \rightarrow \infty$ using the results of $\S 4$ and the fact that $\alpha_{n}\left(\lambda_{n}\right) \rightarrow 0$. Finally let $\hat{x}_{1} \rightarrow \infty$ to obtain

$$
-\int_{I} \rho_{d} \gamma_{d} \frac{w_{x_{1}}}{1+w_{x_{2}}} \leq-(e-3 \varepsilon) \int_{x_{1}}^{\infty} F^{2}(s) d s-K \int_{I} \rho_{d} w f_{1}(\nabla w) .
$$

Here $w$ is the weak limit in $H_{0}^{1}(T)$ of the sequence $\left\{w_{n}\right\}$ and $F$ is defined in analogy with $F_{n}$ in (5.17). Inequality $(5.44)$ can be written as

$$
\int_{I} \rho_{d} \frac{w_{x_{1}}}{1+w_{x_{2}}} \gamma_{d}\left(x_{2}\right)\left\{1-\frac{K w\left(x_{1}, x_{2}\right)}{\gamma_{d}\left(x_{2}\right)}\right\} \geq(e-3 \varepsilon) \int_{x_{1}}^{\infty} F^{2}(s) d s .
$$

Now from the mean value theorem

$$
\left|\frac{w\left(x_{1}, \cdot\right)}{\gamma_{d}(\cdot)}\right|_{L^{\infty}(I)} \leq \tilde{K}\left|w_{x_{2}}\left(x_{1}, \cdot\right)\right|_{L^{\infty}(I)}
$$


and from Theorem 5.1(c) the right-hand side approaches zero as $x_{1} \rightarrow \infty$. It follows from the last two inequalities that for all large $x_{1}$

$$
2 \int_{I} \rho_{d} w_{x_{1}} \gamma_{d} \geq(e-3 \varepsilon) \int_{x_{1}}^{\infty} F^{2}(s) d s .
$$

As in the proof of Theorem 5.4 one concludes from the strong maximum principle that $w_{x_{1}}<0$ in $(0, \infty) \times I$ or that $w \equiv 0$ on $T$. The inequality (5.46) ensures that only the latter can occur, completing the proof of part (a) of Theorem 5.1.

For part (b) recall that $w_{n}$ was taken to converge to $w$ uniformly on compact sets. Since $w_{n}$ has its maximum on the line $x_{1}=0$, the convergence to $w \equiv 0$ is uniform on $T$. Lemma 4.3 and Theorem 4.4 show that $\left|\nabla w_{n}\right| \rightarrow 0$ uniformly in $T$ as $n \rightarrow \infty$. Q.E.D.

THEOREM 5.7. Let $\delta \in(0,1)$ and $R>0$ be fixed and suppose $\left(\lambda_{n}, w_{n}\right) \in F_{n, \delta, R}$ for $n=1,2, \ldots$ Assume $\lambda_{n} \rightarrow \lambda_{d}$ and $w_{n}$ converges weakly to zero in $H_{0}^{1}(T)$ as $n \rightarrow \infty$. Then $w_{n}$ converges strongly to zero in $H_{0}^{1}(T)$.

PROOF. In the previous proof it was shown that the inequality (5.43) holds for $n>N(\varepsilon)$ and $x_{1}>X(\varepsilon)$, the latter restriction being made solely to insure that $w_{n}$ is sufficiently small in $C^{0,1}\left(\left[x_{1}, \infty\right) \times \bar{I}\right)$. From the previous theorem we know that $w_{n}$ approaches zero in $C^{0,1}(\bar{T})$ and thus inequality (5.43) can be shown to hold for all $x_{1} \geq 0$. This will enable us to relate the $H_{0}^{1}$ norm of $w_{n}$ to the quantity $F_{n}(0)$ in such a way as to show that $w_{n}$ approaches zero strongly in $H_{0}^{\mathbf{1}}(T)$ as $F_{n}(0) \rightarrow 0$.

From (5.43) one obtains the two differential inequalities

$$
\left(d / d x_{1}\right)\left\{G_{n}-K U_{n}\right\} \leq-\alpha_{n}\left(\lambda_{n}\right) F_{n}-(e-3 \varepsilon) F_{n}^{2}
$$

and

$$
\left(d / d x_{1}\right)\left\{G_{n}+K U_{n}\right\} \geq-\alpha_{n}\left(\lambda_{n}\right) F_{n}-(e+3 \varepsilon) F_{n}^{2}
$$

valid for $n>N(\varepsilon)$ and for all $x_{1} \geq 0$. As noted earlier, we have

$$
G_{n} \pm K U_{n}=\int_{I} \rho_{n} \frac{\left(w_{n}\right)_{x_{1}}}{1+\left(w_{n}\right)_{x_{2}}} \gamma_{n}\left\{1 \pm \frac{K w_{n}}{\gamma_{n}}\right\}
$$

Since

$$
\left|\frac{w_{n}\left(x_{1}, \cdot\right)}{\gamma_{n}(\cdot)}\right|_{L^{\infty}(I)} \leq \hat{K}\left|\nabla w_{n}\right|_{L^{\infty}(I)}
$$

and $\left|\nabla w_{n}\right|_{L^{\infty}(T)} \rightarrow 0$ as $n \rightarrow \infty, N(\varepsilon)$ may be increased, if necessary, so that for all $n>N(\varepsilon)$

$$
\begin{aligned}
(1+\varepsilon) F_{n}^{\prime} & =(1+\varepsilon) \int_{I} \rho_{n} \gamma_{n}\left(w_{n}\right)_{x_{1}} \leq G_{n} \pm K U_{n} \\
& \leq(1-\varepsilon) \int_{I} \rho_{n} \gamma_{n}\left(w_{n}\right)_{x_{1}}=(1-\varepsilon) F_{n}^{\prime}
\end{aligned}
$$

Recall that $F_{n}^{\prime}$ is negative.

The expression $\alpha_{n}\left(\lambda_{n}\right)+(e+3 \varepsilon) F_{n}\left(x_{1}\right)$ is monotone decreasing on $0 \leq x_{1}<\infty$ and approaches $\alpha_{n}\left(\lambda_{n}\right)<0$ as $x_{1} \rightarrow \infty$. Let $\left[Z_{n}, \infty\right)$ be the subset of $[0, \infty)$ on which the expression is nonpositive. Suppose first that $Z_{n}=0$. If the inequality 
(5.47b) is multiplied by the positive quantity $-\left(G_{n}+K U_{n}\right)$, the result integrated from $x_{1}$ to $\infty$, and use is made of $(5.50)$, then

$$
(1+\varepsilon)^{2}\left(F_{n}^{\prime}\right)^{2} \geq-(1-\varepsilon)\left(\alpha_{n}\left(\lambda_{n}\right) F_{n}^{2}+\frac{2}{3}(e+3 \varepsilon) F_{n}^{3}\right)
$$

Since $F_{n}^{\prime}(0)=0$, it follows from (5.51) that

$$
F_{n}^{2}(0)\left(\alpha_{n}\left(\lambda_{n}\right)+\frac{2}{3}(e+3 \varepsilon) F_{n}(0)\right) \geq 0 .
$$

However, the assumption that $Z_{n}=0$ or that $\alpha_{n}\left(\lambda_{n}\right)+(e+3 \varepsilon) F_{n}(0) \leq 0$ is in contradiction to (5.52), given that $\alpha_{n}\left(\lambda_{n}\right)<0$.

We now know that $Z_{n}>0$ and that

$$
\alpha_{n}\left(\lambda_{n}\right)+\frac{2}{3}(e+3 \varepsilon) F_{n}\left(Z_{n}\right) \leq \alpha_{n}\left(\lambda_{n}\right)+(e+3 \varepsilon) F_{n}\left(Z_{n}\right) \leq 0 .
$$

Using this inequality in (5.51) one finds

$$
(1+\varepsilon)^{2}\left(F_{n}^{\prime}\left(x_{1}\right)\right)^{2} \geq \frac{2}{3}(1-\varepsilon)(e+3 \varepsilon) F_{n}^{2}\left(x_{1}\right)\left(F_{n}\left(Z_{n}\right)-F_{n}\left(x_{1}\right)\right)
$$

on $\left[Z_{n}, \infty\right)$. This last inequality yields

$$
\begin{aligned}
\int_{Z_{n}}^{\infty} F_{n}^{2}\left(x_{1}\right) d x_{1} & =\int_{0}^{F_{n}\left(Z_{n}\right)} \frac{t^{2}}{-F_{n}^{\prime}} d t \\
& \leq K \int_{0}^{F_{n}\left(Z_{n}\right)} \frac{t^{2} d t}{t \sqrt{F_{n}\left(Z_{n}\right)-t}} \\
& \leq K_{1} F_{n}^{3 / 2}\left(Z_{n}\right) \leq K_{1} F_{n}^{3 / 2}(0) .
\end{aligned}
$$

On the interval $\left[0, Z_{n}\right]$,

$$
-\alpha_{n}\left(\lambda_{n}\right) \leq(e+3 \varepsilon) F_{n}\left(x_{1}\right),
$$

and the use of this in (5.47a) yields

$$
\begin{aligned}
\left(d / d x_{1}\right)\left(G_{n}-K U_{n}\right) & \leq(e+3 \varepsilon) F_{n}^{2}-(e-3 \varepsilon) F_{n}^{2} \\
& =6 \varepsilon F_{n}^{2} .
\end{aligned}
$$

We now carry out a process similar to the one above of multiplying (5.56) by $-\left(G_{n}-K U_{n}\right)$, integrating from 0 to $x_{1}$, and using (5.50) to obtain

$$
-\left.\frac{\left(G_{n}-K U_{n}\right)^{2}}{2}\right|_{0} ^{x_{1}} \leq\left. 6 \varepsilon(1+\varepsilon) \frac{F_{n}^{3}}{3}\right|_{0} ^{x_{1}}, \quad x_{1} \in\left[0, Z_{n}\right] .
$$

Now (5.50) can be used to show that $G_{n}-K U_{n}=0$ at $x_{1}=0$ and to bound $\left(G_{n}\left(x_{1}\right)-K U_{n}\left(x_{1}\right)\right)^{2}$ above by $(1+\varepsilon)^{2}\left(F_{n}^{\prime}\left(x_{1}\right)\right)^{2}$. Hence from (5.57) one has

$$
(1+\varepsilon)^{2}\left(F_{n}^{\prime}\left(x_{1}\right)\right)^{2} \geq 4 \varepsilon(1+\varepsilon)\left(F_{n}^{3}(0)-F_{n}^{3}\left(x_{1}\right)\right)
$$

valid for $x_{1} \in\left[0, Z_{n}\right]$. We use this estimate as follows

$$
\begin{aligned}
\int_{0}^{Z_{n}} F_{n}^{2}\left(x_{1}\right) d x_{1} & =\int_{F_{n}\left(Z_{n}\right)}^{F_{n}(0)} \frac{t^{2} d t}{-F_{n}^{\prime}} \\
& \leq K^{\prime} \int_{F_{n}\left(Z_{n}\right)}^{F_{n}(0)} \frac{t^{2} d t}{\sqrt{F_{n}^{3}(0)-t^{3}}} \\
& \leq K_{2} F_{n}^{3 / 2}(0)
\end{aligned}
$$


and combining this with (5.55) yields

$$
\int_{0}^{\infty} F_{n}^{2}\left(x_{1}\right) d x_{1} \leq K_{3} F_{n}^{3 / 2}(0) \text {. }
$$

Now we use inequality (5.41) which, like the inequality (5.43) used at the outset of the proof, will hold on $0 \leq x_{1}<\infty$ provided $n$ is sufficiently large, thereby guaranteeing that $w_{n}$ is sufficiently small in $C^{0,1}(\bar{T})$. From (5.41) it follows that

$$
\int_{0}^{\infty}\left(F_{n}^{\prime}\right)^{2} d x_{1}+\int_{0}^{\infty} \int_{I} \rho_{n}\left|\nabla R_{n}\right|^{2} \leq K_{3} \int_{0}^{\infty} F_{n}^{3} d x_{1}
$$

since $\int_{0}^{\infty} U_{n}^{\prime} d x_{1}=0$. The Poincaré inequality yields

$$
\int_{0}^{\infty} \int_{I} \rho_{n} R_{n}^{2} \leq K_{4} \int_{0}^{\infty} \int_{I} \rho_{n}\left|\nabla R_{n}\right|^{2} .
$$

Now, since $w_{n}$ is even in $x_{1}$ and has the form $w_{n}=F_{n} \gamma_{n}+R_{n}$ (cf. (5.16)-(5.19)), it follows from the last three inequalities that

$$
\left|w_{n}\right|_{H_{0}^{1}(T)}^{2} \leq K\left(F_{n}^{3 / 2}(0)+F_{n}^{5 / 2}(0)\right) .
$$

By Theorem $5.6 F_{n}(0) \rightarrow 0$ as $n \rightarrow \infty$ and hence $w_{n}$ converges strongly to zero in $H_{0}^{1}(T)$. Q.E.D.

The result just completed, together with Corollary 5.3 and Theorem 5.4, yields

THEOREM 5.8. For each $\delta \in(0,1)$ and each $R>0$ the set $\bigcup_{n} F_{n, \delta, R}$ is precompact in $\mathbf{R} \times H_{0}^{1}(T)$.

The concluding result of this section, in which the $H^{1}$ topology still plays the major role, is

THEOREM 5.9. Let $\delta \in(0,1)$ and $R>1$ be fixed. Then

(a) the set

$$
G_{\delta, R}=\underset{n}{\lim \sup } F_{n, \delta, R}
$$

is a compact, connected subset of $B(R)$ (defined in 4.4) with $\left(\lambda_{d}, 0\right) \in G_{\delta, R}$.

(b) Each $(\lambda, w)$ in $G_{\delta, R}$ satisfies parts (a), (c), and (d) of Theorem 5.1 and $\nabla w\left(x_{1}, x_{2}\right) \in Q_{\delta}$ for all $\left(x_{1}, x_{2}\right) \in \overline{T^{-}} \cup \overline{T^{+}}$.

(c) If $(\lambda, w) \in G_{\delta, R} \backslash\left\{\left(\lambda_{d}, 0\right)\right\}$, then $\lambda<\lambda_{d}, w>0$ in $T$, and $\partial w / \partial x_{1}<0$ on $(0, \infty) \times I$.

Proof. (a) From Lemma 4.1 and Theorem 5.8 one can conclude that $G_{\delta, R}$ is a compact, connected set in $B(R)$. It was shown in Lemma 5.5(c) that $\mu_{n} \rightarrow \lambda_{d}$ as $n \rightarrow \infty$ and thus $\left(\lambda_{d}, 0\right) \in G_{\delta, R}$.

Parts (b) and (c) follow from Theorems 5.1, 5.4, 5.6, and 5.7. Q.E.D.

6. The set $G_{\delta, R}$ as a subset of $\mathbf{R} \times\left(H_{0}^{1}(T) \cap C^{0,1}(\bar{T})\right)$. To show the existence of the unbounded, connected set of solutions $S$ described in part (a) of Theorem 2.1 two issues must be confronted. The first is the connectedness of $G_{\delta, R}$ when the stronger topology of $H_{0}^{1}(T) \cap C^{0,1}(\bar{T})$ is used in place of the $H_{0}^{1}$ topology for $w$. The second is roughly to show that in the set $G_{\delta, R}$ there are solutions for which $w$ has size $R$ in $H_{0}^{1}$ or size $\delta^{-1}$ in $C^{0,1}$. If this can be done for any $R>0$ and any $\delta \in(0,1)$, then the unboundedness of $S$ will follow. It will be shown that in $G_{\delta, R}$ 
there is a pair $(\lambda, w)$ for which $|w|_{H^{1}}=R$ or for which $\nabla w\left(\tilde{x}_{1}, \tilde{x}_{2}\right) \in \partial Q_{\delta}$ for some $\left(\tilde{x}_{1}, \tilde{x}_{2}\right)$. This almost resolves the second issue. The final step is to show that for sufficiently small $\delta, \nabla w$ cannot take values on that part of $\partial Q_{\delta}$ where $p_{2}=-1+\delta$.

THEOREM 6.1. For each $\delta \in(0,1)$ and each $R>0$, the set $G_{\delta, R}$ is connected in $\mathbf{R} \times\left(H_{0}^{1}(T) \cap C^{0,1}(\bar{T})\right)$.

Proof. Since $G_{\delta, R}$ is connected in the original topology of $\mathbf{R} \times H_{0}^{1}(T)$, it will suffice to show that on $G_{\delta, R}$ the stronger topology coincides with the original one. That is, if solutions $\left(\lambda_{k}, w_{k}\right)$ of the problem with discontinuous density $\rho_{d}$ converge to $(\lambda, w)$ in $\mathbf{R} \times H_{0}^{1}$, then $w_{k}$ converges to $w$ in $C^{0,1}(\bar{T})$. The Hölder estimates in Theorem 5.1 are satisfied by each $w_{k}$, uniformly in $k$. The compactness of the collection $\left\{w_{k}\right\}$ in $C^{0,1}(\bar{T})$ is then assured and the desired convergence follows immediately. Q.E.D.

Recall that for the density $\rho_{n}$ the set of solutions $\mathcal{E}_{n}$ emanating from $\left(\mu_{n}, 0\right)$ is unbounded in $\mathbf{R} \times H_{0}^{1}(T)$ and that $F_{n, \delta, R}$ is the maximal connected subset of

$$
\left\{(\lambda, w) \in \mathcal{E}_{n} \mid \nabla w\left(x_{1}, x_{2}\right) \in Q_{\delta},\left(x_{1}, x_{2}\right) \in \bar{T}\right\}
$$

which contains $\left(\mu_{n}, 0\right)$ and is contained in $B(R)$. We can assume $n$ is large enough so that $\mu_{n}<2 \lambda_{n}$, and hence for each $n$ there is a pair $\left(\lambda_{n}, w_{n}\right) \in F_{n, \delta, R}$ for which either

(A) $\left|w_{n}\right|_{H_{0}^{1}}=R$, or

(B) $\nabla w_{n}\left(x_{1}^{(n)}, x_{2}^{(n)}\right) \in \partial Q_{\delta}$ for some $\left(x_{1}^{(n)}, x_{2}^{(n)}\right) \in \bar{T}$.

If alternative (A) holds for an infinite sequence of values for $n$, a subsequence, still denoted by $\left(\lambda_{n}, w_{n}\right)$ may be chosen so that $\lambda_{n} \rightarrow \lambda$ and $w_{n}$ converge weakly to $w$ in $H_{0}^{1}(T)$ as $n \rightarrow \infty$. Theorems 5.4 and 5.7 show the convergence to be strong in $H_{0}^{1}$ and hence there is a pair $(\lambda, w)$ in $G_{\delta, R}$ with $|w|_{H_{0}^{1}}=R$.

If alternative (B) occurs infinitely often, it can be assumed that a subsequence is chosen so that the following result is applicable.

THEOREM 6.2. Suppose $\left(\lambda_{n}, w_{n}\right) \in F_{n, \delta, R}, n=1,2, \ldots$, and that for each $n$ there exists a point $\left(x_{1}^{(n)}, x_{2}^{(n)}\right)$ in $\bar{T}$ for which

$$
\nabla w_{n}\left(x_{1}^{(n)}, x_{2}^{(n)}\right) \in \partial Q_{\delta} .
$$

Then there is a pair $(\lambda, w) \in G_{\delta, R}$ and a point $\left(\tilde{x}_{1}, \tilde{x}_{2}\right)$ such that

$$
\nabla w\left(\tilde{x}_{1}, \tilde{x}_{2}\right) \in \partial Q_{\delta}
$$

where, if $\tilde{x}_{2}=0$, the limit from above or below $\tilde{x}_{2}=0$ is intended.

PROOF. The proof is divided into three cases according to which part of the boundary $\partial Q_{\delta}$ is in question. Once again a subsequence can be chosen and renumbered so that $w_{n}$ converges to $w$ in $H_{0}^{1}(T), \nabla w \in Q_{\delta}$ on $\bar{T}$, and one of the following three cases is relevant.

Case 1. $\left|\partial w\left(x_{1}^{(n)}, x_{2}^{(n)}\right) / \partial x_{1}\right|=\delta^{-1} ; n=1,2, \ldots$ First note that Corollary 4.7 guarantees that $\nabla w_{n}$ decays to zero at infinity, uniformly in $n$, so that $\left(x_{1}^{(n)}, x_{2}^{(n)}\right)$ remains bounded. Without loss of generality assume that these points converge to $\left(\tilde{x}_{1}, \tilde{x}_{2}\right)$. From the Hölder estimate $(4.20)$ it is clear that $\partial w_{n} / \partial x_{1}$ converges uniformly to $\partial w / \partial x_{1}$ and thus

$$
\left|\partial w\left(\tilde{x}_{1}, \tilde{x}_{2}\right) / \partial x_{1}\right|=\delta^{-1}
$$

implying (6.2), since $\nabla w \in Q_{\delta}$. 
Case 2. $\partial w_{n}\left(x_{1}^{(n)}, x_{2}^{(n)}\right) / \partial x_{2}=\delta^{-1}, n=1,2, \ldots$ As with Case 1 it can be assumed that $\left(x_{1}^{(n)}, x_{2}^{(n)}\right) \rightarrow\left(\tilde{x}_{1}, \tilde{x}_{2}\right)$. If $\tilde{x}_{2} \neq 0$, the Hölder estimate (4.21) applies to give

$$
\partial w\left(\tilde{x}_{1}, \tilde{x}_{2}\right) / \partial x_{2}=\delta^{-1}
$$

and hence (6.2).

Suppose $\tilde{x}_{2}=0$. From Lemma 4.3 it can be assumed that both $w_{n}$ and $\partial w_{n} / \partial x_{1}$ converge uniformly in a neighborhood $N$ of $\left(\tilde{x}_{1}, 0\right)$. The functions $\partial w_{n} / \partial x_{2}$ cannot, in general, converge uniformly since $\partial w / \partial x_{2}$ is discontinuous along $\left\{x_{2}=0\right\}$. However, from (5.3), the limits of the $x_{2}$ derivatives of $w$ from above and below $\left\{x_{2}=0\right\}$ exist, and we define

$$
w_{x_{2}}^{+}=\lim _{x_{2} \rightarrow 0+} w_{x_{2}}\left(\tilde{x}_{1}, x_{2}\right)
$$

and

$$
w_{x_{2}}^{-}=\lim _{x_{2} \rightarrow 0-} w_{x_{2}}\left(\tilde{x}_{1}, x_{2}\right) .
$$

The link among these various limits in provided by the combination

$$
\rho_{n}\left(x_{2}\right)\left(f_{2}\left(\nabla w_{n}\right)-\lambda_{n} w_{n}\right),
$$

which, according to Theorem 4.4(b), has a Hölder exponent $\alpha>0$, uniformly in $n$. This information combines to give

$$
\begin{gathered}
\lim _{n \rightarrow \infty} \rho_{n}\left(x_{2}^{(n)}\right)\left\{f_{2}\left(w_{x_{1}}, \delta^{-1}\right)-\lambda w\right\} \\
=\rho_{+}\left\{f_{2}\left(w_{x_{1}}, w_{x_{2}}^{+}\right)-\lambda w\right\} \\
=\rho_{-}\left\{f_{2}\left(w_{x_{1}}, w_{x_{2}}^{-}\right)-\lambda w\right\},
\end{gathered}
$$

where the terms other than $w_{x_{2}}$ are evaluated at $\left(\tilde{x}_{1}, 0\right)$.

Now one considers cases. If

$$
f_{2}\left(w_{x_{1}}, \delta^{-1}\right)-\lambda w=0
$$

then the bracketed terms in (6.4) and (6.5) must also vanish, yielding

$$
f_{2}\left(w_{x_{1}}, w_{x_{2}}^{-}\right)=f_{2}\left(w_{x_{1}}, w_{x_{2}}^{+}\right)=f_{2}\left(w_{x_{1}}, \delta^{-1}\right) .
$$

Since $f_{22}>0$, it follows that

$$
w_{x_{2}}^{-}=w_{x_{2}}^{+}=\delta^{-1}
$$

and thus (6.2) holds.

Next, suppose

$$
f_{2}\left(w_{x_{1}}, \delta^{-1}\right)-\lambda w>0
$$

Since $\rho_{+} \leq \rho_{n}\left(x_{2}\right)$ for all $x_{2}$, it follows from (6.3) and (6.4) that

$$
\rho_{+}\left\{f_{2}\left(w_{x_{1}}, \delta^{-1}\right)-\lambda w\right\} \leq \rho_{+}\left\{f_{2}\left(w_{x_{1}}, w_{x_{2}}^{+}\right)-\lambda w\right\}
$$

and so

$$
f_{2}\left(w_{x_{1}}, \delta^{-1}\right) \leq f_{2}\left(w_{x_{1}}, w_{x_{2}}^{+}\right) .
$$

Again using $f_{22}>0$ one concludes that

$$
\delta^{-1} \leq w_{x_{2}}^{+}
$$

and since any limiting values of the gradient of $w$ must lie in $Q_{\delta},(6.2)$ follows. 
If the inequality in (6.6) is reversed, an argument similar to that just given, but using the equality of (6.3) and (6.5), leads to (6.2).

Case 3. $\partial w_{n}\left(x_{1}^{(n)}, x_{2}^{(n)}\right) / \partial x_{2}=-1+\delta ; n=1,2, \ldots$.

The argument here is similar to that given in Case 2, but with $\delta^{-1}$ replaced by $-1+\delta$. Q.E.D.

Our next task is to show that $w_{x_{2}}$ cannot assume the value $-1+\delta$ if $\delta$ is sufficiently small. Since $-1+\delta \leq w_{x_{2}} \leq \delta^{-1}$, the value $-1+\delta$ would be a minimum for $w_{x_{2}}$.

LEMMA 6.3. The minimum of $w_{x_{2}}$ is assumed on the line $\left\{x_{2}=0\right\}$ as a limit from above or below.

PROOF. Since $w$ satisfies the equation

$$
\partial f_{i}(\nabla w) / \partial x_{i}=0 \quad \text { in } T^{+} \cup T^{-},
$$

it follows from differentiation that

$$
\frac{\partial}{\partial x_{i}} f_{i j}(\nabla w) \frac{\partial}{\partial x_{j}} \frac{\partial w}{\partial x_{k}}=0 \quad \text { in } T^{+} \cup T^{-}
$$

for $k=1$ or 2 . By the maximum principle, $w_{x_{2}}$, which is continuous in $\overline{T^{+}}$and in $\overline{T^{-}}$, must take its minimum value on the boundary of $T^{+} \cup T^{-}$. Since $w$ is positive in $T$ and vanishes for $x_{2}=-h$ and for $x_{2}=1-h$, the strong maximum principle implies that $w_{x_{2}}>0$ for $x_{2}=-h$ and $w_{x_{2}}<0$ for $x_{2}=1-h$. Consequently the minimum is negative and must occur where $x_{2}=0$ or $x_{2}=1-h$.

The function $w_{x_{1}}$ is negative in $(0, \infty) \times I^{+}(\mathrm{cf}$. Theorem 5.4) and is zero on the half line $(0, \infty) \times\{1-h\}$. Since $w$ is even in $x_{1}, w_{x_{1}}$ is zero on $\{0\} \times I^{+}$and the strong maximum principle implies

$$
w_{x_{1} x_{2}}>0 \text { on }(0, \infty) \times\{1-h\}
$$

and

$$
w_{x_{1} x_{1}}<0 \text { on }\{0\} \times I^{+} .
$$

The inequality (6.9) implies that the minimum of $w_{x_{2}}$ on the line $x_{2}=1-h$ must occur at $x_{1}=0$. Suppose the equation (6.7) is written out in nondivergence form. Since $w_{x_{1} x_{2}}=0$ on $\{0\} \times I^{+}$, it follows from (6.10) and the ellipticity of (6.7) that

$$
w_{x_{2} x_{2}}>0 \text { on }\{0\} \times I^{+} \text {. }
$$

Hence the minimum of $w_{x_{2}}$ on $\{0\} \times \overline{I^{+}}$must occur at $(0,0)$, showing that the minimum must occur on $\left\{x_{2}=0\right\}$. Q.E.D.

The next result establishes a lower bound for $\lambda$ which serves in Theorem 2.1, part (b) and which will also be used in obtaining a lower bound for $w_{x_{2}}$. The proof of this lemma will be given in connection with related computations at the end of $\S 8$.

LEMMA 6.4. For any $\delta \in(0,1)$ and $R>0$, let $(\lambda, w)$ belong to $G_{\delta, R}$. Then

$$
\lambda \geq \frac{1}{2} \frac{\left(\sqrt{\rho_{-}}+\sqrt{\rho_{+}}\right)^{2}}{\rho_{-}-\rho_{+}} .
$$


Recall that the switch from spatial to semi-Lagrangian independent coordinates was made to circumvent the difficulties inherent in a direct approach to a free boundary. For each solution $(\lambda, w) \in G_{\delta, R}$ the free boundary is merely the graph $\Gamma=\{x, w(x, 0)\}$ of the function $w(x, 0)$. Recall from Theorem 5.9 that $w(\cdot, 0) \in$ $C^{1, \alpha}(\mathbf{R})$. In $\S 7$ it will be shown that it is real-analytic. Having obtained the boundary separating the two fluid components we now revert to the pseudo-streamfunction $\psi(x, y)$, where $(x, y)$ are spatial coordinates in the strip $S$ (cf. $\S 2$ ). Recall from (3.10) that

$$
1+\frac{\partial w}{\partial x_{2}}=\frac{c \sqrt{\rho_{ \pm}}}{\partial \psi / \partial y}
$$

in $S^{+}$, the upper fluid region, and in $S^{-}$, the lower one, respectively. Since $c^{2} \geq$ $g / \lambda_{d},(6.13)$ will give a lower bound for $w_{x_{2}}$ if an upper bound for $\psi_{y}$ is established. The next lemma is a first step towards bounding $\psi_{y}$.

LEMma 6.5. Let $(\lambda, w)$ belong to $G_{\delta, R}$ and let $\psi$ be the corresponding pseudostream-function. Then for any real $m$

$$
\int_{m-1}^{m+1} \int_{I}|\nabla \psi|^{2} d x d y \leq c^{2} K_{1}
$$

where $K_{1}$ depends only on $\rho_{ \pm}$and $h$.

REMARK. From Lemma 6.4 it is clear that $c^{2} K_{1}=g K_{1} / \lambda$ is bounded in terms of $\rho_{ \pm}$and $h$.

Proof OF LeMma 6.5. Let the integral in (6.14) be denoted by $E$. From the relations $(2.20),(2.30),(3.10)$, and (3.11) there results

$$
\begin{aligned}
E & =\int_{m-1}^{m+1} \int_{\Psi(-h)}^{\Psi(1-h)} \frac{1+y_{x}^{2}}{y_{\psi}} d x d \psi \\
& =\int_{m-1}^{m+1} \int_{-h}^{1-h} \frac{1+w_{x_{1}}^{2}}{1+w_{x_{2}}} c \sqrt{\rho_{d}\left(x_{2}\right)} d x_{1} \frac{d \Psi}{d x_{2}} d x_{2} \\
& =c^{2} \int_{m-1}^{m+1} \int_{-h}^{1-h} \rho_{d} \frac{1+w_{x_{1}}^{2}}{1+w_{x_{2}}} d x_{1} d x_{2} .
\end{aligned}
$$

Let $\mu\left(x_{1}\right)$ be a smooth cutoff function which equals 1 on $[m-1, m+1]$ and has support on $[m-2, m+2]$. Consider the fundamental equation (4.1) for a pair $\left(\lambda_{n}, w_{n}\right) \in F_{n, \delta, R}$ converging to $(\lambda, w) \in G_{\delta, R}$. Multiplying (4.1) by $\mu^{2} w_{n}$, integrating by parts, and letting $n \rightarrow \infty$, one obtains (cf. 4.11)

$$
\begin{aligned}
& \frac{1}{2} \int_{T} \rho_{d} \mu^{2}\left(\frac{2+w_{x_{2}}}{\left(1+w_{x_{2}}\right)^{2}}\right)|\nabla w|^{2} \\
& \quad-2 \int_{T} \rho_{d} \mu \mu^{\prime} \frac{w w_{x_{1}}}{1+w_{x_{2}}}+\lambda\left(\rho_{-}-\rho_{+}\right) \int_{-\infty}^{\infty} \mu^{2}\left(x_{1}\right) w^{2}\left(x_{1}, 0\right) d x_{1} \\
& \quad \leq \varepsilon \int_{T} \rho_{d} \mu^{2} \frac{w_{x_{1}}^{2}}{\left(1+w_{x_{2}}\right)^{2}}+\frac{\rho_{-}}{\varepsilon} \int_{m-2}^{m+2}\left(\mu^{\prime}\right)^{2} d x+4 \lambda\left(\rho_{-}-\rho_{+}\right),
\end{aligned}
$$

where $|w| \leq 1$ (cf. 4.35) has been used. If $\varepsilon$ is set equal to $1 / 2$ in (6.16), the resulting inequality implies

$$
\frac{1}{2} \int_{m-1}^{m+1} \int_{I} \rho_{d} \frac{\left(2+w_{x_{2}}\right)}{\left(1+w_{x_{2}}\right)^{2}} w_{x_{2}}^{2}+\frac{1}{2} \int_{m-1}^{m+1} \int_{I} \rho_{d} \frac{w_{x_{1}}^{2}}{1+w_{x_{2}}} \leq K^{\prime}
$$


where $K^{\prime}$ depends only on $\rho_{ \pm}$and $h$. The last integrand in (6.15) contains a sum of $\left(1+w_{x_{2}}\right)^{-1}$ and $w_{x_{1}}^{2}\left(1+w_{x_{2}}\right)^{-1}$. The second of these also occurs in the second integral of (6.17) and so is estimable.

Now consider $\rho_{d}\left(1+w_{x_{2}}\right)^{-1}$. Where $w_{x_{2}} \geq-\frac{1}{2}$, this integrand is at most $2 \rho_{d}$, while on the complementary set

$$
\frac{1}{1+w_{x_{2}}} \leq \frac{4}{3} \frac{2+w_{x_{2}}}{\left(1+w_{x_{2}}\right)^{2}} w_{x_{2}}^{2} .
$$

Use of these estimates with the first integral in (6.17) gives a bound for the integral of $\rho_{d}\left(1+w_{x_{2}}\right)^{-1}$, and the combined estimates yield an estimte for (6.15). Q.E.D.

To make use of the bound (6.14) it will be necessary to show that the distance from the streamline $\Gamma$ to the boundary $\partial S$, denoted $\operatorname{dist}(\Gamma, \partial S)$, is bounded below.

Lemma 6.6. Suppose $(\lambda, w) \in G_{\delta, R}$. Then

$$
\operatorname{dist}(\Gamma, \partial S) \geq K>0,
$$

where $K$ depends only on $\rho_{ \pm}$and $h$.

PROOF. In the case of elevation waves $(e>0)$ being treated, $w \geq 0$ so the distance from $\Gamma$ to the lower boundary is at least $h$. Since $w$ is even in $x_{1}$ and decreasing on $0 \leq x_{1}<\infty$, the distance to the upper boundary is $d=1-h-w(0,0)$.

Consider polar coordinates centered at $(x, y)=(0,1-h)$ so that points within the strip $S$ have angular coordinates in $[0, \pi]$. From Lemma 6.5

$$
\int_{d}^{1} \int_{0}^{\pi}|\nabla \psi|^{2} r d r d \theta \leq \int_{-h}^{1-h} \int_{-1}^{1}|\nabla \psi|^{2} d x d y \leq c^{2} K_{1}
$$

with $K_{1}$ depending only on $\rho_{ \pm}$and $h$. From (6.19)

$$
\int_{d}^{1} \frac{d r}{r} \int_{0}^{\pi}\left|\frac{\partial \psi}{\partial \theta}\right|^{2} d \theta \leq c^{2} K_{1}
$$

For each fixed $r \in(0,1), \psi(r, \theta)=\Psi(1-h)$ when $\theta=0$ or $\pi$, while for each $r \in(d, 1)$ the semicircle of radius $r(0 \leq \theta \leq \pi)$ must intersect $\Gamma$; that is, there must be an angle $\tilde{\theta}=\tilde{\theta}(r)$ in $(0, \pi)$ for which $\psi(r, \tilde{\theta})=0$. Since

$$
\Psi^{2}(1-h)=\left|\int_{\tilde{\theta}}^{0} \frac{\partial \psi}{\partial \theta}(r, \theta) d \theta\right|^{2} \leq \tilde{\theta} \int_{0}^{\tilde{\theta}}\left|\frac{\partial \psi}{\partial \theta}\right|^{2} d \theta \leq \pi \int_{0}^{\pi}\left|\frac{\partial \psi}{\partial \theta}\right|^{2} d \theta
$$

it follows from (6.20) that

$$
\left(\log \frac{1}{d}\right) \frac{\Psi^{2}(1-h)}{\pi} \leq c^{2} K_{1} .
$$

From (1.20), $\Psi(1-h)=c \sqrt{\rho_{+}}(1-h)$ and so

$$
\log \frac{1}{d} \leq \frac{K_{1} \pi}{\rho_{+}(1-h)^{2}},
$$

from which (6.18) follows. Q.E.D.

The following lemma of Alt, Caffarelli, and Friedman $[\mathbf{1}]$ will be used. 
LEMMA 6.7. Let $u$ be a function in $C\left(B_{a}\right) \cap W^{1,2}\left(B_{a}\right)$, where $B_{r} \subset \mathbf{R}^{2}$ is a ball of radius $r$ centered at $(\tilde{x}, \tilde{y})$, where $u(\tilde{x}, \tilde{y})=0$. Suppose $u$ is harmonic in $B_{a} \backslash\{u=0\}$ and set

$$
\varphi(r)=\frac{1}{r^{2}} \int_{B_{r}}\left|\nabla u^{+}\right|^{2} d x d y \cdot \frac{1}{r^{2}} \int_{B_{r}}\left|\nabla u^{-}\right|^{2} d x d y
$$

where $u^{+}=\sup (u, 0)$ and $u^{-}=\inf (u, 0)$. Then $\varphi(r)$ is an increasing function of $r$ for $0<r<a$.

THEOREM 6.8. Suppose $(\lambda, w) \in G_{\delta, R}$. Then

$$
\left|\frac{1}{1+w_{x_{2}}}\right|_{L^{\infty}(T)} \leq K_{1}
$$

and

$$
\left|\frac{w_{x_{1}}}{1+w_{x_{2}}}\right|_{L^{\infty}(T)} \leq K_{2},
$$

where the constants depend only on $\rho_{ \pm}$and $h$.

PROOF. In Lemma 6.3 it was shown that the minimum of $1+w_{x_{2}}$ is assumed on the line $\left\{x_{2}=0\right\}$, from above or below. From the discussion surrounding (6.13) an upper bound for $\partial \psi / \partial y$ on the upper and lower sides of $\Gamma$ will yield (6.24). The estimate (6.25) follows similarly from a bound on $\partial \psi / \partial x$ which is harmonic in $S^{ \pm}$ and must assume its maximum and minimum on $\partial S^{+} \cup \partial S^{-}$. Since $\psi$ is constant on the lines $\{y=-h\}$ and $\{y=1-h\}$, it suffices to bound $\left|\psi_{x}\right|$ on $\Gamma$.

Let $\psi^{+}$denote the restriction of $\psi$ to $S^{+}$and $\psi^{-}$, the restriction to $S^{-}$. Since $\psi^{+}>0$ in $S^{+}$and $\psi^{-}<0$ in $S^{-}$, this notation is consistent with that in the previous lemma. According to Theorem 5.1 the function $w(x, 0)$ describing $\Gamma$ is of class $C^{1, \alpha}$ and $\nabla w$ is of class $C^{\alpha}$ in $\overline{T^{+}}$. Since $\nabla w$ lies in $Q_{\delta}$, the relations (3.10) and (3.11) show that $\nabla \psi^{ \pm}$is of class $C^{\alpha}$ in $\overline{S^{ \pm}}$, respectively. Let $(\tilde{x}, \tilde{y})$ be a point on $\Gamma$ and let $\sigma^{ \pm}=\left|\nabla \psi^{ \pm}(\tilde{x}, \tilde{y})\right|$. If $B_{r}$ denotes the ball of radius $r$ centered at $(\tilde{x}, \tilde{y})$, then from the previous lemma

$$
\varphi(r)=\frac{1}{r^{2}} \int_{B_{r} \cap S^{+}}\left|\nabla \psi^{+}\right|^{2} \cdot \frac{1}{r^{2}} \int_{B_{r} \cap S^{-}}\left|\nabla \psi^{-}\right|^{2}
$$

is increasing in $r$ for $0<r<a$ where $a=\min (h, \operatorname{dist}(\Gamma, \partial S))$. From the regularity just cited it follows that $\varphi(r)$ has a limit, denoted $\varphi(0)$, as $r \rightarrow 0$ and

$$
\frac{\pi}{2}\left(\sigma^{+}\right)^{2} \cdot \frac{\pi}{2}\left(\sigma^{-}\right)^{2}=\varphi(0)<\varphi(a) \leq\left(a^{-2} c^{2} K_{1}\right)^{2}
$$

by Lemma 6.5 .

Recall that the continuity of $\rho_{d}\left(f_{2}(\nabla w)-\lambda w\right)$ (cf. 4.31) is a translation of the pressure condition (2.28) which becomes

$$
\frac{1}{2}\left[\left(\sigma^{+}\right)^{2}-\left(\sigma^{-}\right)^{2}\right]=\left(\rho_{+}-\rho_{-}\right)\left\{c^{2} / 2-g \tilde{y}\right\}
$$

in the current notation. The two relations (6.27) and (6.28) easily imply that $\sigma^{+}$ and $\sigma^{-}$are bounded in terms of $\rho_{ \pm}$and $h$. Q.E.D.

It follows from the last result that there is a $\delta_{0}>0$ so that for $0<\delta<\delta_{0}$ and $R>0$ there is a pair $(\lambda, w) \in G_{\delta, R}$ for which

$$
|w|_{H_{0}^{1}(T)}=R
$$


or

$$
\max \left(\left|w_{x_{1}}\right|_{L^{\infty}(T)},\left|w_{x_{2}}\right|_{L^{\infty}(T)}\right)=\delta^{-1}
$$

Hence $S \subset \mathbf{R} \times\left(H_{0}^{1}(T) \cap C^{0,1}(T)\right)$ defined by

$$
S=\bigcup_{n} G_{1 / n, n}
$$

is an unbounded, connected set of solutions of equations (2.36)-(2.39).

\section{Proof of Theorem 2.1.}

7.1. Existence and basic properties. The major part of the work involved in proving Theorem 2.1 has been done. In this section we summarize those results already obtained and add proofs for the remaining parts of the theorem, with one exception. As already noted, the lower bound $\underline{\lambda}$ for $\lambda$, given by (6.12), is derived in $\S 8$.

Part (a) The basic existence result in Theorem 2.1 was the culmination of the analysis in $\S \S 3-6$ and was asserted at the end of the previous section. That elements of $S$ are solutions of (2.36)-(2.39) follows from Theorem 5.1(d). The inclusion of $\left(\lambda_{d}, 0\right)$ in $S$ follows from Lemma $5.5(\mathrm{c})$.

Part (b) The inequality $\lambda \leq \lambda_{d}$ was given in Theorem 5.1. From Theorem 5.6 it follows that $\left(\lambda_{d}, w\right) \in S$ implies $w \equiv 0$. All functions $w$ under consideration have been even in $x_{1}$ and hence their limits are even in $x_{1}$. The remaining parts of (b) were shown in Theorem 5.4 .

Part (c) The analyticity of $w$ in the closed strips $\overline{T^{ \pm}}$will be shown in Theorem 7.6 below. As for the other assertions of regularity, they follow from Theorem 5.1.

Part (d) These bounds, which translate into bounds on velocities, were obtained in Theorem 6.8.

Part (e) In Lemma 7.4 of [26] the exponential decay of solutions was shown both for periodic waves over a half period and for solitary waves. The proof in [26] can be simplified if only solitary waves are considered.

Part (f) This nonexistence result is shown in Theorem 7.7 below.

In the event $e<0$, one can use (5.43) to arrive at the reversal of inequality (5.46) and so prove Theorem 5.6. Similar small changes are required for the proof of Theorem 5.7.

7.2. Analyticity of the interface. As was observed by Kinderlehrer, Nirenberg, and Spruck [15] the analyticity of $w$ in $\overline{T^{ \pm}}$will follow from results of Morrey [20; 21, Chapter 6] once it is shown that $w$ is of class $C^{2}$ in $\overline{T^{ \pm}}$. In [15] solutions of free boundary problems are examined under a typical assumption that the solution is known to be $C^{2}$. Then a partial hodograph or Legendre transformation together with suitable combinations and reflections of solutions in abutting domains produce an elliptic system adapted to Morrey's results. Here we have worked in a semiLagrangian (partial hodograph) setting for most of the analysis and hence need only do a reflection once the $C^{2}$ character of $w$ in $\overline{T^{ \pm}}$is established. The $C^{2}$ estimates could be carried out using the techniques from [3], but a relatively short direct proof can be given and we do that in Corollary 7.5. A lemma is required (and could be given in a local version). 
LEMMA 7.1. Let $(\lambda, w)$ be an element of $G_{\delta, R}$ (cf. (4.7)). Then there is a $p=p(\delta)>2$ and a constant $K$ depending on $\delta$ and $R$ such that

$$
\int_{T} v_{x_{i}}^{4} \leq K\left\{1+\left(\int_{T}\left|\nabla v_{x_{1}}\right|^{2}\right)^{2-p / 2}\right\}
$$

for $i=1,2$, where $v=w_{x_{1}}$.

PROOF. It will suffice to work with an element $(\lambda, w) \in F_{n, \delta, R}$ (see $\S 4$ ) and obtain an estimate which is independent of $n$. The subscript $n$ will be suppressed.

The function $v$ satisfies

$$
-\frac{\partial}{\partial x_{i}}\left(\rho f_{i j}(\nabla w) \frac{\partial v}{\partial x_{j}}\right)=-\lambda \rho^{\prime} v \quad \text { in } T
$$

and

$$
v=0 \text { on } \partial T \text {. }
$$

From inequalities (4.9) and (4.29) it follows that there is a $p=p(\delta)>2$ such that

$$
|\nabla v|_{L^{*}(T)} \leq K|w|_{L^{2}(T)} \leq K^{\prime} R
$$

for all $s$ in the interval $[2, p(\delta)]$, where $K$ and $K^{\prime}$ depend on $\delta$. A version of the Gagliardo-Nirenberg inequality will be needed for a function $u \in H^{1}(T)$. By extending $u$ as an even function across the lines $\left\{x_{2}=-h\right\}$ and $\left\{x_{2}=1-h\right\}$ and using a cutoff function in the $x_{2}$ direction, one easily derives

$$
|u|_{L^{4}(T)} \leq K_{1}|u|_{H^{1}(T)}^{1-p / 4}|u|_{L^{p}(T)}^{p / 4}
$$

from the main theorem in lecture II of [22]. Here $p$ can take any value in the interval $[1,4]$. We can, without loss of generality, assume $p(\delta)<4$ and let $p=p(\delta)$.

Equation (7.2) can be written as

$$
\begin{gathered}
\frac{\partial}{\partial x_{2}}\left(\rho f_{21} \frac{\partial v}{\partial x_{1}}+\rho f_{22} \frac{\partial v}{\partial x_{2}}-\lambda \rho v\right)=-\frac{\partial}{\partial x_{1}}\left(\rho f_{1 j} \frac{\partial v}{\partial x_{j}}\right)-\lambda \rho \frac{\partial v}{\partial x_{2}} \\
=-\rho\left\{f_{1 j k} \frac{\partial v}{\partial x_{j}} \frac{\partial v}{\partial x_{k}}+f_{1 j} \frac{\partial \gamma}{\partial x_{j}}\right\}-\lambda \rho \frac{\partial v}{\partial x_{2}},
\end{gathered}
$$

where

$$
\gamma=\frac{\partial v}{\partial x_{1}}=\frac{\partial^{2} w}{\partial x_{1}^{2}}
$$

$f_{i j k}$ denotes a third derivative, and the summation convention is used. Without using the equation one has

$$
\begin{aligned}
\frac{\partial}{\partial x_{1}} & \left(\rho f_{21} \frac{\partial v}{\partial x_{1}}+\rho f_{22} \frac{\partial v}{\partial x_{2}}-\lambda \rho v\right) \\
& =\rho\left\{f_{21 k} \frac{\partial v}{\partial x_{1}} \frac{\partial v}{\partial x_{k}}+f_{21} \frac{\partial \gamma}{\partial x_{1}}+f_{22 k} \frac{\partial v}{\partial x_{2}} \frac{\partial v}{\partial x_{k}}+f_{22} \frac{\partial \gamma}{\partial x_{2}}-\lambda \gamma\right\} .
\end{aligned}
$$

With $\rho f_{2 j} v_{x_{j}}-\lambda \rho v$ denoted by $u$, it follows from (7.6) and (7.8) that

$$
\int_{T}|\nabla u|^{2} \leq K_{2}\left[\int_{T}\left(|\nabla \gamma|^{2}+|\nabla v|^{2}+v_{x_{1}}^{4}+v_{x_{2}}^{4}\right)\right]
$$


where $K_{2}$ depends on $\delta$ and $R$. Since the $L^{2}$ norm of $|\nabla v|$ and, by embedding, any $L^{p}$ norm of $v$ are bounded in terms of $R$ (cf. (4.9)), such terms can be accommodated by including additive or multiplicative constants. Since $f_{22}$ is bounded below by a positive constant depending on $\delta$, the use of (7.5) yields

$$
\begin{aligned}
\int_{T} v_{x_{2}}^{4} & \leq K_{3} \int_{T}\left[u^{4}+\left(\rho f_{21} \frac{\partial v}{\partial x_{1}}-\lambda \rho v\right)^{4}\right] \\
& \leq K_{4} \int_{T} u^{4}+K_{1}^{\prime}\left(\int_{T} v^{4}+\int_{T} v_{x_{1}}^{4}\right) \\
& \leq K_{5}\left[|\nabla u|_{L^{2}(T)}^{4-p}|u|_{L^{p}(T)}^{p}+1+\int_{T} v_{x_{1}}^{4}\right] .
\end{aligned}
$$

Using (7.4) to estimate $u$ in $L^{p}$ and (7.9) to estimate $\nabla u$ in $L^{2}$, we have

$$
\int_{T} v_{x_{2}}^{4} \leq K_{6}\left[1+\int_{T} v_{x_{1}}^{4}+\left\{\int_{T}\left(|\nabla \gamma|^{2}+v_{x_{1}}^{4}+v_{x_{2}}^{4}\right)\right\}^{2-p / 2}\right] .
$$

Since $2-p / 2<1$, equation $(7.11)$ provides a bound

$$
\int_{T} v_{x_{2}}^{4} \leq K_{7}\left[1+\int_{T} v_{x_{1}}^{4}+\left\{\int_{T}|\nabla \gamma|^{2}\right\}^{2-p / 2}\right],
$$

where $K_{7}$ depends only on $\delta$ and $R$.

The embedding inequality (7.5) applied to $v_{x_{1}} \in H_{0}^{1}(T)$, gives

$$
\int_{T} v_{x_{1}}^{4} \leq K_{8}\left\{\int_{T}|\nabla \gamma|^{2}\right\}^{2-p / 2} \int_{T}\left|v_{x_{1}}\right|^{p} \leq K_{9}\left\{\int_{T}|\nabla \gamma|^{2}\right\}^{2-p / 2},
$$

again using $p=p(\delta)$ and (7.4). The use of (7.13) alone, and in (7.12) produces the result (7.1). Q.E.D.

THEOREM 7.2. Suppose $(\lambda, w) \in G_{\delta, R}$. Then

$$
\int_{T}\left|\nabla w_{x_{1} x_{1}}\right|^{2} \leq K
$$

where $K$ depends on $\delta$ and $R$.

ProOF. Continuing the analysis in the previous proof for a solution in $F_{n, \delta, R}$, one differentiates (7.2) to obtain

$$
-\frac{\partial}{\partial x_{i}}\left(\rho f_{i j}(\nabla w) \frac{\partial \gamma}{\partial x_{j}}\right)=\frac{\partial}{\partial x_{i}}\left(\rho f_{i j k} \frac{\partial v}{\partial x_{j}} \frac{\partial v}{\partial x_{k}}\right)-\lambda \rho^{\prime} \gamma \quad \text { in } T
$$

and, since $\gamma=\partial^{2} w / \partial x_{1}^{2}$,

$$
\gamma=0 \quad \text { on } \partial T \text {. }
$$

The ellipticity of the equation (7.15) yields

$$
\begin{aligned}
\nu \int_{T}|\nabla \gamma|^{2} & \leq \int_{T} \rho f_{i j} \frac{\partial \gamma}{\partial x_{i}} \frac{\partial \gamma}{\partial x_{j}} \\
& =-\int_{T} \rho \frac{\partial \gamma}{\partial x_{i}} f_{i j k} \frac{\partial v}{\partial x_{j}} \frac{\partial v}{\partial x_{k}}+2 \lambda \int_{T} \rho \gamma \gamma_{x_{2}} \\
& \leq \frac{\nu}{2} \int_{T}|\nabla \gamma|^{2}+K_{1} \int_{T}\left(v_{x_{1}}^{4}+v_{x_{2}}^{4}+\gamma^{2}\right) .
\end{aligned}
$$


The bound (7.14) now follows from the previous lemma and Lemma 4.2. We note again that the estimates are all uniform in $n$ and so hold in $G_{\delta, R}$. Q.E.D.

COROLlaRY 7.3. If $(\lambda, w) \in G_{\delta, R}$, then

$$
\left|\nabla w_{x_{1}}\right|_{L^{p}(T)} \leq K
$$

for all $p \in[2, \infty)$, where $K$ depends only on $p, \delta$, and $R$.

PROOF. From the previous theorem the integral of $|\nabla \gamma|^{2}$ over $T$ is bounded and from Lemma 7.1, so are the integrals of $v_{x_{1}}^{4}$ and $v_{x_{2}}^{4}$. From (7.9)

$$
\int_{T}\left|\nabla\left(\rho f_{21} \frac{\partial v}{\partial x_{1}}+\rho f_{22} \frac{\partial v}{\partial x_{2}}-\lambda \rho v\right)\right|^{2} \leq K_{1},
$$

where $K_{1}$ depends upon $\delta$ and $R$. Hence $\rho f_{2 i} v_{x_{i}}-\lambda \rho v$ is in $L^{p}(T)$ for all $p \in[2, \infty)$, according to embedding theory. The same embedding result, used with (4.9) and Theorem 7.2, shows that $v$ and $v_{x_{1}}$ are in $L^{p}(T)$ for all $p \in[2, \infty)$. Since $f_{22}$ has a positive lower bound depending only on $\delta$, it follows that $v_{x_{2}}$ is in the same $L^{p}$ spaces. Q.E.D.

It is now possible to return to the elliptic equation for $\gamma$ to obtain

THEOREM 7.4. Let $(\lambda, w)$ be in $G_{\delta, R}$. Then there is a $q=q(\delta)>2$ so that $\gamma=w_{x_{1} x_{1}}$ satisfies

$$
\int_{T}|\nabla \gamma|^{q} \leq K
$$

where $K$ depends on $\delta$ and $R$.

ProOF. For $(\lambda, w) \in F_{n, \delta, R}$ the equation (7.15) for $\gamma=w_{x_{1} x_{1}}$ can be written

$$
-\frac{\partial}{\partial x_{i}}\left(\rho f_{i j} \frac{\partial \gamma}{\partial x_{j}}\right)=\operatorname{div} F+h,
$$

where

$$
F_{1}=\rho f_{i j k} \frac{\partial v}{\partial x_{j}} \frac{\partial v}{\partial x_{k}}, \quad F_{2}=\rho f_{2 j k} \frac{\partial v}{\partial x_{j}} \frac{\partial v}{\partial x_{k}}-\lambda \rho \gamma
$$

and

$$
h=\lambda \rho \frac{\partial \gamma}{\partial x_{2}} .
$$

By the previous corollary $F_{1}$ and $F_{2}$ are in $L^{p}(T)$ fer $p \in[2, \infty)$, while from Theorem $7.2, h \in L^{2}(T)$. As for equation (4.24), Theorem 1 of Meyers [18] yields (7.19) for some $q>2$. All estimates are independent of $n$ so the inequality (7.19) follows for all $(\lambda, w) \in G_{\delta, R}$. Q.E.D.

Corollary 7.5. Let $(\lambda, w)$ be in $G_{\delta, R}$. Then $w \in C^{2, \alpha}\left(\overline{T^{ \pm}}\right)$for some $\alpha>0$ and hence the fluid interface $y=w(x, 0)$ is of class $C^{2, \alpha}$.

PROOF. The $C^{2, \alpha}$ character of the interface follows from (7.19) and embedding theory. The Schauder theory $[13$, Lemma 6.18] provides the same regularity in $\overline{T \pm}$. Q.E.D. 
THEOREM 7.6. Suppose $(\lambda, w)$ is a solution of (2.36)-(2.39) belonging to $S$. Then $w$ is real-analytic in $\overline{T^{-}}$and $\overline{T^{+}}$.

ProOF. Since $w$ is of class $C^{2, \alpha}$ in $\overline{T^{ \pm}}$, the techniques of [15] can now be used. In fact, if we revert to the formulation in which $\psi(x, y)$ is harmonic in $S^{+}$and $S^{-}$ and satisfies $(2.27),(2.28)$, then Theorem 3.2 of $[\mathbf{1 5}]$ yields analyticity.

7.3. Nonexistence of small solutions with $e \cdot w<0$. We now prove part (f) of Theorem 2.1.

THEOREM 7.7. Suppose

$$
e=\rho_{-} / h^{2}-\rho_{+} /(1-h)^{2}>0 .
$$

There is a positive $\eta$ such that if $(\lambda, w) \in \mathbf{R} \times\left(H_{0}^{1}(T) \cap C^{0,1}(\bar{T})\right)$ is a solution of (2.39) with $w \leq 0, \partial w / \partial x_{1} \geq 0$ for $x_{1} \geq 0$, and

$$
\left|\lambda-\lambda_{d}\right|+|w|_{H_{0}^{1}(T)}+|w|_{C^{0,1}(\bar{T})}<\eta
$$

then $w \equiv 0$.

PROOF. Repeating estimates already used, in conjunction with difference quotients in the $x_{1}$ direction, one can show $w$ is smooth in each of $\overline{T^{-}}$and $\overline{T^{+}}$, and we shall assume the smoothness necessary for the calculations to follow. First it will be shown that if the hypotheses of the theorem are met and $\lambda>\lambda_{d}$, then $w \equiv 0$. The steps involved in the proof of Theorem 5.6 up to (5.40) can be duplicated for the case of a discontinuous density, that is, for $\rho_{d}$. The equation (5.12) which formally becomes

$$
\left(\rho_{d} \gamma^{\prime}\right)^{\prime}-\lambda \rho_{d}^{\prime} \gamma=\alpha \rho_{d} \gamma
$$

is replaced by the weak form

$$
-\int_{I} \rho_{d} \gamma^{\prime} \varphi^{\prime}+\lambda \int_{I} \rho_{d}(\gamma \varphi)^{\prime}=\alpha \int_{I} \rho_{d} \gamma \varphi,
$$

where $\gamma$ and $\varphi$ are in $H_{0}^{1}(I)$. Equation (7.21) arises from the variational problem

$$
\alpha=\max _{u \in H_{0}^{1}(I)} \frac{\left\{\lambda\left(\rho_{-}-\rho_{+}\right) u^{2}(0)-\int_{I} \rho_{d}\left(u^{\prime}\right)^{2}\right\}}{\int_{I} \rho_{d} u^{2}},
$$

which corresponds to (5.11). The maximizer $\gamma$ is normalized by $\int \rho_{d} \gamma^{2}=1$ and, as in the proof of Lemma 5.5, we have $\alpha>0$ since $\lambda>\lambda_{d}$. In following the proof of Theorem 5.6 one need only let the derivative of a density in an integral be interpreted as

$$
\int_{I} \rho_{d}^{\prime} g\left(x_{1}, x_{2}\right) d x_{2}=-\left(\rho_{-}-\rho_{+}\right) g\left(x_{1}, 0\right)
$$

in the case of the discontinuous $\rho_{d}$ from (2.26). As regards (5.35), let $\tau$ correspond to the density $\rho_{d}$. The extra condition of orthogonality yields $\tau>\lambda_{d}$, and it can be assumed that $\lambda$ is sufficiently close to $\lambda_{d}$ to make $1-\lambda / \tau=\theta>0$.

As regards the steps in the proof of Theorem 5.6 past (5.40), the only change is in the discussion following (5.40), for now $\alpha$ is positive rather than negative. Inequality (5.41) is replaced by

$$
\left(F^{\prime}\right)^{2}+\int_{I} \rho_{d}|\nabla R|^{2} \leq \frac{2 \alpha}{\theta} F^{2}+\frac{2}{\theta} U^{\prime}+K^{\prime} F^{3}
$$


and in place of (5.42) one has

$$
G^{\prime}+\alpha F+e F^{2} \geq-2 \varepsilon F^{2}-K^{\prime \prime}\left(\frac{2 \alpha}{\theta} F^{2}+\frac{2}{\theta} U^{\prime}+K^{\prime} F^{3}\right) .
$$

It follows from this inequality that at each $x$

$$
G^{\prime}-K U^{\prime}+\left(\alpha-K_{1}|F|_{L_{x}^{\infty}}\right) F \geq 0
$$

for constants $K$ and $K_{1}$, where $|F|_{L_{x}^{\infty}}=\max _{x \leq s<\infty}|F(s)|$. The last inequality, integrated from $x \geq 0$ to $\infty$ yields (cf. $(5.45)$ )

$$
-\int_{I} \rho_{d} \gamma \frac{w_{x_{1}}\left(x, x_{2}\right)}{1+w_{x_{2}}\left(x, x_{2}\right)}\left\{1-\frac{K w}{\gamma}\right\} d x_{2}+\left(\alpha-K_{1}|F|_{L_{x}^{\infty}}\right) \int_{x}^{\infty} F(s) d s \geq 0 .
$$

If $w$ is small in $C^{0,1}(\bar{T})$ and $x$ is large, both $1-K w / \gamma$ and $\alpha-K_{1}|F|_{L_{x}^{\infty}}$ will be positive. Since $w_{x_{1}} \geq 0$ and $F \leq 0, w \equiv 0$ for $x$ large. However, $w$ is analytic in $T^{ \pm}$so $w \equiv 0$.

Now we may assume $\lambda \leq \lambda_{d}$. If $\varphi$ is set equal to $w$ in the equation (2.39), the result is

$$
\int_{T} \rho_{d} \frac{|\nabla w|^{2}\left(2+w_{x_{2}}\right)}{2\left(1+w_{x_{2}}\right)^{2}}=\int_{T} \rho_{d} f_{i}(\nabla w) w_{x_{i}}=\lambda\left(\rho_{-}-\rho_{+}\right) \int_{-\infty}^{\infty} w^{2}\left(x_{1}, 0\right) d x_{1}
$$

or, rewritten,

$$
\int_{T} \rho_{d}|\nabla w|^{2}-\lambda\left(\rho_{-}-\rho_{+}\right) \int_{-\infty}^{\infty} w^{2}\left(x_{1}, 0\right) d x_{1}=\int_{T} \rho_{d}|\nabla w|^{2} \frac{w_{x_{2}}\left(3+2 w_{x_{2}}\right)}{2\left(1+w_{x_{2}}\right)^{2}}
$$

In analogy with (5.16)-(5.18), we decompose $w$ as

$$
w\left(x_{1}, x_{2}\right)=F\left(x_{1}\right) \gamma_{d}\left(x_{2}\right)+R\left(x_{1}, x_{2}\right),
$$

where $\gamma_{d}$ is the function described in (5.13) and

$$
F\left(x_{1}\right)=\int_{I} \rho_{d} w\left(x_{1}, x_{2}\right) \gamma_{d} d x_{2}, \quad x_{1} \in \mathbf{R} .
$$

Note that $\gamma_{d}$ satisfies the weak equation

$$
\int_{I} \rho_{d} \gamma_{d}^{\prime} \varphi^{\prime}=\lambda_{d}\left(\rho_{-}-\rho_{+}\right) \gamma_{d}(0) \varphi(0)
$$

for any $\varphi \in H_{0}^{1}(I)$. A further relation, the analogue of (5.19), is

$$
\int_{I} \rho_{d} R\left(x_{1}, x_{2}\right) \gamma_{d} d x_{2}=0, \quad x_{1} \in \mathbf{R} .
$$

The decomposition (7.23) and the relations (7.24)-(7.25), used in the left-hand side of $(7.22)$, result in

$$
\begin{aligned}
\int_{T} \rho_{d}|\nabla w|^{2}-\lambda\left(\rho_{-}-\rho_{+}\right) \int_{-\infty}^{\infty} w^{2}\left(x_{1}, 0\right) d x_{1} \\
=\int_{-\infty}^{\infty}\left(F^{\prime}\right)^{2} d x_{1}+\int_{T} \rho_{d}|\nabla R|^{2}-\lambda\left(\rho_{-}-\rho_{+}\right) \int_{-\infty}^{\infty} R^{2}\left(x_{1}, 0\right) d x_{1} \\
\quad+\left(\rho_{-}-\rho_{+}\right) \gamma_{d}^{2}(0)\left(\lambda_{d}-\lambda\right) \int_{-\infty}^{\infty} F^{2} d x_{1} \\
\quad+2\left(\lambda_{d}-\lambda\right)\left(\rho_{-}-\rho_{+}\right) \gamma_{d}(0) \int_{-\infty}^{\infty} F R\left(x_{1}, 0\right) d x_{1} .
\end{aligned}
$$


In analogy with the discussion following (5.35), the orthogonality (7.25) ensures that

$$
\int_{T} \rho_{d}|\nabla R|^{2}-\lambda\left(\rho_{-}-\rho_{+}\right) \int_{-\infty}^{\infty} R^{2}\left(x_{1}, 0\right) d x_{1} \geq K \int_{T}|\nabla R|^{2}
$$

for some $K>0$ whenever $\left|\lambda-\lambda_{d}\right|$ is sufficiently small. For the last term in (7.26) we use the Schwarz and Poincaré inequalities to obtain

$$
\begin{aligned}
& -2\left(\lambda_{d}-\lambda\right)\left(\rho_{-}-\rho_{+}\right) \gamma_{d}(0) \int_{-\infty}^{\infty} F\left(x_{1}\right) R\left(x_{1}, 0\right) d x \\
& \quad \leq \frac{K}{2} \int_{T}|\nabla R|^{2}+K^{\prime}\left|\lambda_{d}-\lambda\right|^{2} \gamma_{d}^{2}(0) \int_{-\infty}^{\infty} F^{2} d x_{1} .
\end{aligned}
$$

If (7.27) and (7.28) are used in (7.26) and if $\lambda_{d}-\lambda$ is made still smaller if necessary, there results

$$
\int_{T} \rho_{d}|\nabla w|^{2}-\lambda\left(\rho_{-}-\rho_{+}\right) \int_{-\infty}^{\infty} w^{2}\left(x_{1}, 0\right) d x_{1} \geq \int_{-\infty}^{\infty}\left(F^{\prime}\right)^{2} d x_{1}+\frac{K}{2} \int_{T}|\nabla R|^{2}
$$

Now consider the right-hand side of (7.22). First, for any $\varepsilon>0$,

$$
\int_{T} \rho_{d} w_{x_{1}}^{2} \frac{w_{x_{2}}\left(3+2 w_{x_{2}}\right)}{2\left(1+w_{x_{2}}\right)^{2}} \leq \varepsilon \int_{T}|\nabla R|^{2}+\varepsilon \int_{-\infty}^{\infty}\left(F^{\prime}\right)^{2} d x_{1}
$$

provided $w$ is sufficiently small in $C^{0,1}(\bar{T})$. Next, with the use of (7.23) one obtains (7.31)

$$
\int_{T} \rho_{d} w_{x_{2}}^{2} \frac{w_{x_{2}}\left(3+2 w_{x_{2}}\right)}{2\left(1+w_{x_{2}}\right)^{2}} \leq \int_{T} \rho_{d} \cdot \frac{3}{2}\left(F\left(x_{1}\right) \gamma^{\prime}\left(x_{2}\right)\right)^{3}+K^{\prime} \int_{-\infty}^{\infty} F^{4} d x_{1}+\frac{K}{4} \int_{T}|\nabla R|^{2}
$$

for $w$ sufficiently small in $C^{0,1}(\bar{T})$, where $K$ is the constant appearing in (7.29). The first term on the right-hand side of (7.31) is the integral over $\mathbf{R}$ of $\tilde{e} F^{3}\left(x_{1}\right)$, where $\tilde{e}$ is defined by (5.26) and is positive, being a positive multiple of $e$ in (2.41). If the inequalities (7.29), (7.30), and (7.31) are used with equation (7.22) and the integrand $F^{4}$ is estimated above by $-|F|_{L^{\infty}} F^{3}$, there results

$$
\left(\frac{K}{4}-\varepsilon\right) \int_{T}|\nabla R|^{2}+(1-\varepsilon) \int_{-\infty}^{\infty}\left(F^{\prime}\right)^{2} d x_{1}-\left(\tilde{e}-K^{\prime}|F|_{L^{\infty}}\right) \int_{-\infty}^{\infty} F^{3} d x_{1} \leq 0
$$

Since $F=\int_{I} \rho_{d} w \gamma_{d} \leq 0$, it is clear from the estimates just completed that if $w$ has a sufficiently small norm in $H_{0}^{1}(T) \cap C^{0,1}(\bar{T})$ and if $\left|\lambda-\lambda_{d}\right|$ is sufficiently small, $F \equiv|\nabla R| \equiv 0$ and hence $w \equiv 0$. Q.E.D.

8. Waves of large amplitude. Since the branch of solutions $S$ from Theorem 2.1 is unbounded in $\mathbf{R} \times\left(H_{0}^{1}(T) \cap C^{0,1}(\bar{T})\right)$ and the range of $\lambda$ is bounded, the norms of $w$ in $H_{0}^{1}(T)$ and in $C^{0,1}(\bar{T})$ cannot both remain bounded on $S$.

In $\S 6$ it was established that there is a $\delta_{0}>0$ so that $w_{x_{2}} \geq-1+\delta_{0}$ for all $(\lambda, w) \in S$. If the gradient of $w$ were uniformly bounded for $(\lambda, w) \in S$, there would be a positive $\delta_{1} \leq \delta_{0}$ such that for all solutions in $S, \nabla w \in Q_{\delta_{1}}$. But then the coefficients $f_{i j}$ would satisfy the bounds (4.15) and (4.16) with $\delta=\delta_{1}$, uniformly for $(\lambda, w) \in S$. This, in turn, would imply that in $S$ there would be solutions having $|w|_{L^{2}(T)}$ arbitrarily large. For, if not, then the local estimates (4.20) and (4.33), summed over $m$, would lead to a bound for $\nabla w$ in $L^{2}(T)$ and hence to a bound for 
$w$ in $H_{0}^{1}(T)$. To go one step further, since $0 \leq w \leq 1$, a large norm for $w$ in $L^{2}$ can arise only by having a wave which is very "broad." The alternatives, then, are to have waves which are arbitrarily broad or ones with arbitrarily large gradients. Of course, waves may exhibit both properties.

Numerical computations in the related problem of the interface between two unbounded fluids of differing densities (cf. $[\mathbf{1 7}, \mathbf{2 8}]$ ) show that there are branches of periodic waves along which the gradients approach infinity. The condition (6.25) necessitates $w_{x_{2}}$ being large if the vector $\nabla w$ is large. However, in the computations cited $w_{x_{1}}$ goes to infinity and thus both components of $\nabla w$ must grow unboundedly along the branch. In fact, the streamlines steepen to the point of having vertical tangents and continue past this configuration to one of "overhanging" waves, in which the separating streamline is no longer a graph over the horizontal axis. Computations are underway on the solitary wave problem studied here (cf. [29]), and they show surges occur in certain parameter ranges. We will show that if waves broaden indefinitely, maintaining uniformly bounded gradients, then a solution representing an "internal surge" with specified amplitude and speed (determined by (8.23) and (8.24)) must exist. If, on the other hand, there were a sequence $\left(\lambda_{k}, w_{k}\right) \in S$, $k=1,2, \ldots$, with $\sup \left|\nabla w_{k}\right| \rightarrow \infty$ as $k \rightarrow \infty$, one could expect these to converge to a limiting wave with a vertical tangent on the interface.

8.1. Internal surges. Let $\left(\lambda_{k}, w_{k}\right) \in S, k=1,2$, be a sequence of solutions of (2.36)-(2.39) for the density $\rho_{d}$. Suppose that for some $\delta>0, \nabla w_{k} \in Q_{\delta}$ for all $k$ and that $\left|w_{k}\right|_{L^{2}(T)} \rightarrow \infty$ as $k \rightarrow \infty$. As already noted, the Hölder estimates (4.20) and (4.33) for the gradients will hold uniformly in $k$. Consequently one can use the diagonal method to select a subsequence, still denoted $\left(\lambda_{k}, w_{k}\right)$, for which $\lambda_{k} \rightarrow \hat{\lambda} \leq \lambda_{d}$ and $w_{k}$ converges in $C^{0,1}(\bar{B})$, for all bounded sets $B$, to a function $\hat{w}\left(x_{1}, x_{2}\right) \in C^{0,1}(\bar{T}) \cap C^{1, \alpha}\left(\overline{T^{ \pm}}\right)$. The limiting function $\hat{w}$ will satisfy all of the estimates derived in Lemma 4.3 and Theorem 4.4; namely, the local estimates which are independent of $R$. Naturally, $\hat{w}$ inherits the evenness in $x_{1}$ and satisfies $\partial \hat{w} / \partial x_{1} \leq 0$ for $x_{1} \geq 0$. Since $\hat{w}$ is also nonnegative,

$$
\lim _{x_{1} \rightarrow \infty} \hat{w}\left(x_{1}, x_{2}\right)=\hat{w}_{\infty}\left(x_{2}\right)
$$

exists. To examine further the implication of this convergence, we shift it to $B=$ $[-1,1] \times \bar{I}$ by letting $\hat{w}_{m}\left(x_{1}, x_{2}\right)=\hat{w}\left(x_{1}+m, x_{2}\right)$. Now $\hat{w}_{m}$ is bounded in $C^{1, \alpha}(B)$ uniformly in $m$, according to the Hölder estimates (4.20) and (4.33). Since $\hat{w}_{m}$ converges in $C(B)$ to $\hat{w}_{\infty}$, it must also converge n $C^{0,1}\left(B \cap \bar{T}^{ \pm}\right)$according to the Arzela-Ascoli theorem, and the limit $\hat{w}_{\infty}$ must be in $C^{1, \alpha}\left(\bar{I}^{ \pm}\right)$as a function of $x_{2}$ alone.

Now let $\beta\left(x_{1}\right)$ be an arbitrary element of $C_{0}^{\infty}(-1,1)$ and $\tau$ an arbitrary element of $C_{0}^{\infty}(I)$. Using the product $\beta \tau$ as a test function in (2.39) with $w=\hat{w}_{m}$ and letting $m \rightarrow \infty$, one obtains

$$
\int_{I} \rho_{d}\left(x_{2}\right) f_{2}\left(0, \frac{d \hat{w}_{\infty}}{d x_{2}}\right) \frac{d \tau}{d x_{2}}=\hat{\lambda}\left(\rho_{-}-\rho_{+}\right) \hat{w}_{\infty}(0) \tau(0),
$$

which is the weak form of

$$
\frac{d}{d x_{2}} \rho_{d} f_{2}\left(0, \frac{d \hat{w}_{\infty}}{d x_{2}}\right)=\hat{\lambda} \rho_{d}^{\prime} \hat{w}_{\infty}
$$


As a consequence the nonnegative, continuous function $\hat{w}_{\infty}$ will be linear where $\rho_{d}^{\prime}=0$, that is, on $I^{-}$and on $I^{+}$.

Suppose $\hat{w}_{\infty} \equiv 0$. That is, suppose that $\hat{w} \rightarrow 0$ as $x_{1} \rightarrow \infty$. Then as $x_{1} \rightarrow \infty$ the functions $w_{k}$ defined at the outset of this discussion must converge to zero uniformly in $k$ as elements of $L^{\infty}(I)$ depending on $x_{1}$. Their gradients must converge to zero in the same sense, according to the local estimates (4.20) and (4.33). Suppose $\lambda_{k}$ from the pair $\left(\lambda_{k}, w_{k}\right)$ converges to $\hat{\lambda}<\lambda_{d}$ as $k \rightarrow \infty$. In Lemma 5.2 an estimate was made of the "tail" of $w$; that is, the restriction of $w$ to $T_{m}=[m, \infty) \times I$. There a bound for $w$ in $H_{0}^{1}(T)$ was used to show that the $C^{0,1}\left(\bar{T}_{m}\right)$ norm of $w$ went to zero with $m$, yielding the estimate (5.6). Since the functions $w_{k}$ under discussion have their norms in $C^{0,1}\left(\bar{T}_{m}\right)$ converging to zero with increasing $m$, uniformly in $k$, the estimate (5.6) can be seen to hold for $w=w_{k}$ with the constant $K$ in (5.6) depending only on $\hat{\lambda}$ and on the $\delta$ which serves for all $k$. A bound for $w_{k}$ in $H^{1}([-m, m] \times I)$ follows easily from the estimates in $\S 4$, and thus the $w_{k}$ are uniformly bounded in $H_{0}^{1}(T)$, contradicting the initial hypothesis that $\left|w_{k}\right|_{H_{0}^{1}(T)} \rightarrow$ $\infty$ as $k \rightarrow \infty$.

In the case that $\lambda_{k} \rightarrow \hat{\lambda}=\lambda_{d}$ as $k \rightarrow \infty$ a similar situation prevails as regards the behavior of $w$ and $\nabla w$ for large $x_{1}$. It suffices in the proofs of Theorem 5.6 and 5.7 to have the norm of $w$ in $C^{0,1}\left(\bar{T}_{m}\right)$ approach zero as $m \rightarrow \infty$, and this we have for $w=w_{k}$, uniformly in $k$. A consequence of those theorems is that $\hat{w}=0$ and that $w_{k}$ converges to $\hat{w}=0$ in $H_{0}^{1}(T)$, again contradicting the initial assumptions. Consequently $\hat{w}_{\infty}$ and $\hat{w}$ are both positive. We summarize what has been proven in

THEOREM 8.1. Suppose that $\left(\lambda_{k}, w_{k}\right) \in S, k=1,2, \ldots$, satisfy

(a) $\left|\nabla w_{k}\right|_{L^{\infty}(T)} \leq K$, for all $k$ and

(b) $\lambda_{k} \rightarrow \hat{\lambda}$ and $\left|w_{k}\right|_{H_{0}^{1}(T)} \rightarrow \infty$ as $k \rightarrow \infty$.

Then $w_{k}$ converges to $\hat{w}\left(x_{1}, x_{2}\right)$ in $C(\bar{B}) \cap C^{1}\left(\bar{B} \cap \overline{T^{ \pm}}\right)$for each bounded set $B$. The function $\hat{w}$ is positive on $T$, nonincreasing for $x_{1} \geq 0$, real-analytic in $\bar{T}-\left\{x_{2}=0\right\}$, and satisfies

$$
\lim _{x_{1} \rightarrow \pm \infty} \hat{w}\left(x_{1}, \cdot\right)=\hat{w}_{\infty}
$$

in $C(\bar{I}) \cap C^{0,1}\left(\overline{I^{\mp}}\right)$, where $\hat{w}_{\infty}$ is positive on $I$, linear on $I^{+}$and $I^{-}$, and satisfies (8.2). Both $\hat{w}$ and $\hat{w}_{\infty}$ satisfy (2.36), (2.37), and (2.39).

Note that $\hat{w}_{\infty}$ is a solution of the flow equations (just enumerated) which is independent of $x$. Using the nomenclature of Benjamin [9] we shall call $\hat{w}_{\infty}$ a conjugate flow. According to Theorem 8.1 the function $\hat{w}_{\infty}$ must have the form

$$
\hat{w}_{\infty}\left(x_{2}\right)= \begin{cases}A\left(x_{2}+h\right), & x_{2} \in I^{-}, \\ B\left(x_{2}-1+h\right), & x_{2} \in I^{+}\end{cases}
$$

for some value of $A$ where, by continuity $A h=B(-1+h)$. Equation (8.2) gives $\hat{\lambda}=\Lambda(A)$, where

$$
\Lambda(A)=\frac{1}{\left(\rho_{-}-\rho_{+}\right)} \frac{1}{2 A h}\left[\rho_{-}\left(1-\frac{1}{(1+A)^{2}}\right)-\rho_{+}\left(1-\frac{1}{(1+B)^{2}}\right)\right] .
$$


Since $\partial \hat{w}_{\infty} / \partial x_{2}=B>-1$ on $I^{+}$, we have $0<A<(1-h) / h$ in (8.6). The amplitude $A$ and the speed

$$
c=c_{A}=(g / \Lambda(A))^{1 / 2}
$$

are as yet unknown. To determine $A$ we show that as a consequence of Theorem 8.1 there is a flow in the strip which connects a trivial flow to a conjugate flow. That is, there is a solution of (2.36), (2.37), and (2.39) which has the following asymptotic behavior. As $x \rightarrow-\infty$ it approaches a flow with velocity vector $\left(c_{A}, 0\right)$ in a fluid which has density $\rho_{-}$for $-h<y<0$ and density $\rho_{+}$for $0<y<1-h$. As $x \rightarrow+\infty$ the conditions approached are

$$
\text { velocity }= \begin{cases}\left(\frac{1}{1+A} c_{A}, 0\right) & \text { for }-h<y<A h \\ \left(\frac{(1-h)}{(1-h-A h)} c_{A}, 0\right) & \text { for } A h<y<1-h,\end{cases}
$$

according to the relations (2.43).

THEOREM 8.2. Suppose the hypotheses of Theorem 8.1 are satisfied. Then there is a solution $\left(\hat{\lambda}, j\left(x_{1}, x_{2}\right)\right)$ of $(2.36),(2.37)$, and (2.39) with the following properties:

(a) $j$ is real analytic in $\bar{T}-\left\{x_{2}=0\right\}$ and is in $C^{1, \alpha}\left(\overline{T^{ \pm}}\right) \cap C^{0,1}(\bar{T})$ for some $\alpha>0$.

(b) $\partial j / \partial x_{1}>0$ in $T$.

The element $j\left(x_{1}, \cdot\right)$ of $C^{0,1}(\bar{I})$ satisfies

(c) $\lim _{x_{1} \rightarrow-\infty}\left|j\left(x_{1}, \cdot\right)\right|_{C^{0,1}(\bar{I})}=0$, and

(d) $\lim _{x_{1} \rightarrow+\infty}\left|j\left(x_{1}, \cdot\right)-w_{\infty}\right|_{C^{0,1}(\bar{I})}=0$, where $w_{\infty}$ is a conjugate flow corresponding to some value of $A>0$ and $\hat{\lambda}=\Lambda(A)$.

PROOF. It can be readily verified that

$$
\Lambda(A)=\lambda_{d}-\frac{3 h e}{2\left(\rho_{-}-\rho_{+}\right)} A+O\left(A^{2}\right)
$$

for $A$ near zero, where $\lambda_{d}$ and $e$ are defined by (2.40) and (2.41), respectively. Since $e>0$, by assumption, $\Lambda(A)<\lambda_{d}$ for small positive $A$. Since $\Lambda(A) \rightarrow+\infty$ as $A \rightarrow(1-h) / h$, it is clear that $\Lambda(A)$ is not one-to-one on the preimage of the interval $\left[0, \lambda_{d}\right]$, the set of interest to us. Let the conjugate flow $\hat{w}_{\infty}$ in Theorem 8.1 correspond to a value $\hat{A}>0$ in (8.5). From the form of $\Lambda(A)$ it is clear that there is a smallest positive value $A$ for which $\Lambda(A)=\Lambda(\hat{A})$. Let $w_{\infty}$ in the statement of the theorem correspond to this smallest value.

Return now to the sequence $w_{k}$ from Theorem 8.1. The functions $w_{k}$ converge in $C^{0,1}$ on bounded sets to $\hat{w}$, and $\hat{w}\left(x_{1}, 0\right)$ approaches $\hat{A} h>0$ as $x_{1} \rightarrow \infty$. For each $k$ there is a value $\xi_{k}<0$ (precisely one since $\partial w_{k} / \partial x_{1}>0$ for $x_{1}<0$ ) for which

$$
w_{k}\left(\xi_{k}, 0\right)=\frac{1}{2} A h \leq \frac{1}{2} \hat{A} h .
$$

Define $j_{k}, k=1,2, \ldots$, by

$$
j_{k}\left(x_{1}, x_{2}\right)=w_{k}\left(x_{1}+\xi_{k}, x_{2}\right) .
$$

As with the functions $w_{k}$ one finds that a subsequence of the $j_{k}$, denoted by the same symbols, converges in $C^{0,1}(\bar{B})$, for each bounded $B \subset \bar{T}$, to a function $j\left(x_{1}, x_{2}\right)$ 
in $C^{1, \alpha}\left(\overline{T^{ \pm}}\right) \cap C^{0,1}(\bar{T})$. Since for each fixed $x_{1}, w_{k}\left(x_{1}, 0\right)$ converges to $\hat{w}\left(x_{1}, 0\right) \geq$ $\hat{w}_{\infty}(0)=\hat{A} h$, the points $\xi_{k}$ at which (8.10) holds must approach $-\infty$ as $k \rightarrow \infty$. Again fix a value of $x_{1}$. For all sufficiently large $k, x_{1}+\xi_{k}<0$ and so $\partial j_{k} / x_{1}>0$ at that $x_{1}$ for all $x_{2} \in I$. Thus $\partial j / \partial x_{1}\left(x_{1}, x_{2}\right) \geq 0$ for all $\left(x_{1}, x_{2}\right) \in T$. Since $j$ is monotone, bounded, and in $C^{1, \alpha}\left(\overline{T^{ \pm}}\right) \cap C^{0,1}(\bar{T})$, the limits

$$
j_{ \pm}\left(x_{2}\right)=\lim _{x_{1} \rightarrow \pm \infty} j\left(x_{1}, x_{2}\right)
$$

exist. As in the proof of Theorem 8.1 one shows that the limits can be taken in the $C^{0,1}(\bar{I})$ topology and that $j_{ \pm}$are conjugate flows satisfying $(8.2)$ for $\hat{\lambda}=\Lambda(A)=$ $\Lambda(\hat{A})$.

Since $j_{+}(0) \geq j(0,0)=\frac{1}{2} A h>0$, the function $j_{+}$is a nontrivial conjugate flow. Since $j_{-}(0) \leq j(0,0)=\frac{1}{2} A h$ and since $A h$ is the smallest height at $x_{2}=0$ for a nontrivial conjugate flow with $\lambda=\hat{\lambda}=\Lambda(\hat{A}), j_{-}$must be zero. As claimed the function $j$ then represents an internal "hydraulic jump" connecting a trivial flow at $x=-\infty$ with speed $c$ given by (8.7) to a conjugate flow at $x=+\infty$ with the structure (8.8). The remaining regularity in (i) and the positivity in (ii) are shown as in Theorem 8.1. Q.E.D.

Implicit in the form of the conjugage flow (8.5) is the conservation of mass in the lower fluid, connecting a flow of height $(1+A) h$ and speed $c_{A} /(1+A)$ at $x=+\infty$ with a flow at $-\infty$ of height $h$ and speed $c_{A}$. A similar situation prevails in the upper fluid. This same conservation can be obtained starting with

$$
\operatorname{div}(\rho \mathbf{q})=0
$$

from $\S 2$ (cf. (2.9)-(2.10)) since streamlines are integral curves for the field $\mathbf{q}$.

Another conserved quantity can be derived from the momentum equation (2.8). It can be expressed by the exactness of the form

$$
\rho^{1 / 2} U d \psi+p d y
$$

assuming now that we are working with a smooth density $\rho$. To see the exactness, express (8.14) as

$$
\rho^{1 / 2} U \frac{\partial \psi}{\partial x} d x+\rho^{1 / 2} U \frac{\partial \psi}{\partial y} d y+p d y=-\rho U V d x+\left(\rho U^{2}+p\right) d y .
$$

By using $\operatorname{div} \mathbf{q}=0$ and $\nabla \rho \cdot \mathbf{q}=0$, one may show that

$$
(-\rho U V)_{y}-\left(\rho U^{2}+p\right)_{x}=-\rho\left(U_{x} U+U_{y} V\right)-p_{x}=0,
$$

using the horizontal component of the momentum equation (2.8).

Now $\psi$ and $y$ are constant on the "walls" at $y=-h$ and $y=1-h$, and if (8.14) is integrated around the boundary of the rectangle $[a, b] \times[-h, 1-h]$ there results

$$
\left.\int_{-h}^{1-h}\left(\rho^{1 / 2} U \frac{\partial \psi}{\partial y}+p\right)\right|_{x=a} d y=\left.\int_{-h}^{1-h}\left(\rho^{1 / 2} U \frac{\partial \psi}{\partial y}+p\right)\right|_{x=b} d y
$$

That is, each integral appearing in (8.15) is independent of the $x$ coordinate (cf. [8]). If the expression (2.15) for the total head $H$ is used to replace $p$ in (8.15) and the relation (2.14) is used, the $x$-invariant quantity in (8.15) can be expressed as

$$
\int_{-h}^{1-h}\left[H+\frac{1}{2}\left(\psi_{y}^{2}-\psi_{x}^{2}\right)-g \rho y\right] d y
$$


Given the regularity and the convergence properties of the flows considered up to this point, it is easy to verify that the expression (8.16) will be invariant for the flow associated with $j\left(x_{1}, x_{2}\right)$. By equating the values of (8.16) at $x_{1}=-\infty$ and $x_{1}=+\infty$, one obtains another condition on $A$ in the conjugate flow $j_{+}$, from which $A$ can be determined.

Normalize the pressure so that it is 0 at $x_{1}=-\infty, x_{2}=0$. Then $H$ has the values

$$
H_{ \pm}=\rho_{ \pm} c^{2} / 2
$$

in the upper and lower fluids, respectively. As $x_{1}=x \rightarrow \pm \infty, \psi_{x} \rightarrow 0$ for the flow corresponding to $j$, and the term $\psi_{y}^{2}$ is merely the density times the square of the horizontal velocity. At $x=-\infty$ the expression (8.16) is

$$
\frac{1}{2} \rho_{-} c^{2} h+\frac{1}{2} \rho_{-} c^{2} h+g \rho_{-} h^{2} / 2+\frac{1}{2} \rho_{+} c^{2}(1-h)+\frac{1}{2} \rho_{+} c^{2}(1-h)-g \rho_{+}(1-h)^{2} / 2
$$

where $c=c_{A}$ in (8.7). The value of (8.16) at $x=+\infty$ is computed using the velocities and heights in (8.8). The result is

$$
\begin{aligned}
& \frac{1}{2} \rho_{-} c^{2}(1+A) h+\frac{1}{2} \rho_{-} \frac{c^{2} h}{1+A}-g \rho_{-} \frac{\left(A^{2} h^{2}-h^{2}\right)}{2} \\
& \quad+\frac{1}{2} \rho_{+} c^{2}(1-h-A h)+\frac{1}{2} \rho_{+} \frac{c^{2}(1-h)^{2}}{1-h-A h}-g \rho_{+}\left[\frac{(1-h)^{2}-A^{2} h^{2}}{2}\right] .
\end{aligned}
$$

Considering the expression (8.19) as a power series in $A$, there is a constant term which is, of course, (8.18). The linear term in $A$ vanishes and what remains is

$$
\frac{1}{2} \rho_{-} c^{2} h \frac{A^{2}}{1+A}-g \rho_{-} \frac{A^{2} h^{2}}{2}+\frac{1}{2} \rho_{+} c^{2} \frac{1}{1-h} \frac{A^{2} h^{2}}{(1-A h /(1-h))}+g \rho_{+} \frac{A^{2} h^{2}}{2}
$$

which must be zero. If an $A^{2}$ is cancelled and $g / c^{2}$ set equal to $\hat{\lambda}$, the result is

$$
\hat{\lambda}=\frac{1}{\left(\rho_{-}-\rho_{+}\right)}\left(\frac{\rho_{-}}{h(1+A)}+\frac{\rho_{+}}{1-h-A h}\right) .
$$

If this is equated to the expression (8.6) and terms of zeroth order in $A$ are eliminated, the result is

$$
\frac{\rho_{-} A}{2 h(1+A)^{2}}-\frac{\rho_{+} A h}{2(1-h-A h)^{2}}=0 .
$$

If an $A$ is cancelled and the result solved, one finds

$$
A=\frac{(1-h)}{\sqrt{\rho_{-}}+\sqrt{\rho_{+}}}\left(\frac{\sqrt{\rho_{-}}}{h}-\frac{\sqrt{\rho_{+}}}{1-h}\right)=\frac{(1-h)}{\left(\sqrt{\rho_{-}}+\sqrt{\rho_{+}}\right)} \cdot \frac{e}{\left(\sqrt{\rho_{-}} / h+\sqrt{\rho_{+}} /(1-h)\right)},
$$

where $e$ is the parameter defined in (2.41).

If the expression for $A$ in (8.23) is used in (8.21), the result is

$$
\hat{\lambda}=\frac{\left(\sqrt{\rho_{-}}+\sqrt{\rho_{+}}\right)^{2}}{\rho_{-}-\rho_{+}} \text {. }
$$

This last expression is strictly less than $\lambda_{d}$ when $e \neq 0$ and is equal to $\lambda_{d}$ when $e=0$. 
Next we give the

8.2. PROOF OF LEMMA 6.4. For a smooth density $\rho$ approximating $\rho_{d}$ and a solution $(\lambda, w)$ of $(2.36)-(2.38)$, the equation $(2.36)$ can be written

$$
-\rho\left(x_{2}\right)\left(\frac{w_{x_{1} x_{1}}}{1+w_{x_{2}}}+w_{x_{1}} \frac{\partial}{\partial x_{1}} \frac{1}{\left(1+w_{x_{2}}\right)}\right)-\frac{\partial}{\partial x_{2}} \rho f_{2}(\nabla w)=-\lambda \rho^{\prime} w .
$$

For each $x_{2}, w\left(x_{1}, x_{2}\right)$ has its maximum at $x_{1}=0$ and hence $w_{x_{1}}\left(0, x_{2}\right)=0$ and $w_{x_{1} x_{1}}\left(0, x_{2}\right) \leq 0$. If the last equation is restricted to the line $\left\{x_{1}=0\right\}$, then

$$
-\left(\partial / \partial x_{2}\right) \rho\left(x_{2}\right) f_{2}\left(0, w_{x_{2}}\left(0, x_{2}\right)\right) \leq-\lambda \rho^{\prime}\left(x_{2}\right) w\left(0, x_{2}\right)
$$

results. If $(8.25)$ is multiplied by $g\left(x_{2}\right)=w\left(0, x_{2}\right)$ and the result integrated over $I$, it yields

$$
\int_{I} \rho f_{2}\left(0, g^{\prime}\right) g^{\prime} \leq-\lambda \int_{I} \rho^{\prime} g^{2}
$$

Consider now a pair $(\tilde{\lambda}, \tilde{w}) \in G_{\delta, R}$. There is a sequence $\left(\lambda_{n}, w_{n}\right) \in F_{n, \delta, R}$ with $\lambda_{n} \rightarrow \tilde{\lambda}$ and $w_{n} \rightarrow \tilde{w}$ in $C^{0,1}\left([-1,1] \times \bar{I}_{\varepsilon}\right)$ as $n \rightarrow \infty$ and (8.26) holds with $\rho=\rho_{n}$, $g=g_{n}=w_{n}\left(0, x_{2}\right)$, and $\lambda=\lambda_{n}$. If $F=F(p)$ is any real function defined for $p>-1$ satisfying

$$
F(p) \leq f_{2}(0, p) p
$$

for $-1+\delta \leq p \leq \delta^{-1}$, then it follows that

$$
\lambda_{\delta}=\inf _{\substack{g \in H_{0}^{1}(I) \\-1+\delta \leq g^{\prime} \leq \delta^{-1} \\ 0<g(0) \leq 1-h}} \frac{\int_{I} \rho_{d} F\left(g^{\prime}\right)}{\left(\rho_{-}-\rho_{+}\right) g^{2}(0)}
$$

is a lower bound for $\tilde{\lambda}$. Note that $g(0)=\tilde{w}(0,0)>0$ for any nontrivial element of $G_{\delta, R}$ for the maximum principle applied in $T^{+}$and in $T^{-}$shows $w$ has its maximum at $(0,0)$.

The function $F(p)=f(0, p)$ is easily seen to satisfy (8.27) and moreover, is convex for $p>-1$, having a second derivative equal to $(1+p)^{-3}$. Let $f_{\delta}(p)$ be strictly convex for all $p \in \mathbf{R}$, coincide with $f(0, p)$ for $-1+\delta<p<\delta^{-1}$, and have quadratic growth near $p= \pm \infty$. One can, for example, extend $f_{22}(0, p)$ continuously outside of $\left[-1+\delta, \delta^{-1}\right]$ to be constant for $p<-1+\delta$ and for $p>\delta^{-1}$. Two integrations will then yield a suitable $f_{\delta}$. The infimum in (8.28) will be unaffected by replacing $F\left(g^{\prime}\right)=f\left(0, g^{\prime}\right)$ by $f_{\delta}\left(g^{\prime}\right)$. After replacement the infimum can only be made lower by removing the condition on $g^{\prime}$. Thus

$$
\lambda_{\delta}=\inf _{\substack{g \in H_{0}^{1}(I) \\ 0<g(0) \leq 1-h}} \frac{\int_{I} \rho_{d} f_{\delta}\left(g^{\prime}\right)}{\left(\rho_{-}-\rho_{+}\right) g^{2}(0)}
$$

is a lower bound for $\tilde{\lambda}$.

For each constant $\gamma>0$ the set

$$
Q=\left\{g \in H_{0}^{1}(I) \mid g(0)=\gamma\right\}
$$

is an affine subspace of $H_{0}^{1}(I)$, and the convex functional

$$
J(g)=\int_{I} \rho_{d} f_{\delta}\left(g^{\prime}\right)
$$


is therefore weakly lower semicontinuous on $Q$. Since $J$ is nonnegative, there is a $g_{0} \in Q$ at which $J$ assumes its minimum. For $\eta \in C_{0}^{\infty}\left(I^{-}\right), g_{0}+t \eta \in Q$ and a standard variational argument shows

$$
\int_{I} \rho_{d} f_{\delta}^{\prime}\left(g_{0}^{\prime}\right) \eta^{\prime}=0
$$

From the classical theorem of DuBois-Reymond it follows that $g_{0}^{\prime}$ is constant on $I^{-}$. Likewise it is constant on $I^{+}$. Thus $g_{0}$ has the form (8.5) of a conjugate flow with $g_{0}(0)=A h \leq(1-h)$. For such a function the quotient in (8.29) takes the form

$$
\frac{\frac{1}{2} \rho_{-} A^{2} h /(1+A)+\frac{1}{2} \rho_{+} A^{2} h^{2} /(1-h-A h)}{\left(\rho_{-}-\rho_{+}\right) A^{2} h^{2}}
$$

which is precisely half the expression in (8.21). At a minimum $A$ satisfies (8.22), and the corresponding minimum value is half of that in (8.24), giving the bound (6.12). Q.E.D.

A better lower bound for $\tilde{\lambda}$ could be found. The function

$$
f_{2}(0, p) p=\frac{p^{2}(2+p)}{2(1+p)^{2}}
$$

is convex on $-1+\delta \leq p \leq 2$ for each $\delta \in(0,1)$ and can be extended outside that range, as before, to a globally defined convex function $\tilde{f}_{\delta}(p)$ which grows quadratically at $\infty$. One can now use an argument like that just given. The expression in (8.31) can be used for $F(p)$ in (8.28) with the restriction $-1+\delta \leq$ $g^{\prime} \leq 2$. In (8.29) one replaces $f_{\delta}$ by $\tilde{f}_{\delta}$ and finds a piecewise linear minimizer $\tilde{g}$ having a derivative

$$
\tilde{g}^{\prime}\left(x_{2}\right)= \begin{cases}A>0, & x_{2}<0 \\ -A h /(1-h), & x_{2}>0\end{cases}
$$

Note that $A \in(0,(1-h) / h)$ since $\tilde{g}^{\prime}>-1$. If $h \geq 1 / 3$, so that $(1-h) / h \leq 2$, then $\tilde{g}^{\prime}$ lies in the range for which the integrand in (8.29) is the expression in (8.31). Corresponding to (8.30) one obtains an expression to minimize for $A \in(0,(1-h) / h)$. Unfortunately, the minimizing value of $A$ satisfies a fourth-order equation. Without giving the details we merely note that if $\rho_{-}=2, \rho_{+}=1$, and $h=1 / 2$, so that $(1-h) / h<2$, the following numerical values are obtained. The bifurcation point is $\lambda_{d}=6$, and Lemma 6.4 gives the lower bound 2.91, whence $2.91<\lambda<6$. The minimization using (8.31) yields the better estimate: $5.81<\lambda<6$.

\section{REFERENCES}

1. H. W. Alt, L. A. Caffarelli, and A. Friedman, Variational problems with two phases and their free boundaries, Trans. Amer. Math. Soc. 282 (1984), 431-461.

2. 248.

3. S. Agmon, A. Douglas, and L. Nirenberg, Estimates near the boundary for solutions of elliptic partial differential equations satisfying general boundary conditions. II, Comm. Pure Appl. Math. 17 (1964), 35-92.

4. C. J. Amick, Semilinear elliptic eigenvalue problems on an infinite strip with an application to stratified fluids, Ann. Scuola Norm. Sup. Pisa Sér. (4) 11 (1984), 441-499.

5. C. J. Amick and J. F. Toland, On solitary water-waves of finite amplitude, Arch. Rational Mech. Anal. 76 (1981), 9-95. 
6. J. T. Beale, The existence of solitary water waves, Comm. Pure Appl. Math. 30 (1977), 373-389.

7. H. Beirão da Veiga, R. Serapioni, and A. Valli, On the motion of nonhomogeneous fluids in the presence of diffusion, J. Math. Anal. Appl. 85 (1982), 179-191.

8. T. B. Benjamin, Internal waves of finite amplitude and permanent form, J. Fluid Mech. 25 (1966), 241--270.

9. $587-643$.

10. J. L. Bona, D. K. Bose, and R. E. L. Turner, Finite amplitude steady waves in stratified fluids, J. Math. Pure Appl. 62 (1983), 389-439.

11. A. D. Frank-Kamenetskii, Diffusion and heat transfer in chemical kinetics, 2nd ed., Plenum, New York-London, 1969 [translated from Russian].

12. K. O. Friedrichs and D. H. Hyers, The existence of solitary waves, Comm. Pure Appl. Math. 7 (1954), 517-550.

13. D. Gilbarg and N. S. Trudinger, Elliptic partial differential equations, Academic Press, New York, 1968.

14. A. V. Kazhikhov and Sh. Smagulov, The correctness of boundary value problems in a diffusion model of inhomogeneous liquid, Soviet Phys. Dokl. 22 (1977), 249-250 (Dokl. Akad. Nauk SSSR 234 (1977), 330-332 [in Russian]).

15. D. Kinderlehrer, L. Nirenberg, and J. Spruck, Regularity in elliptic free boundary problems. I, J. Analyse Math. 34 (1978), 86-119.

16. R. R. Long, Solitary waves in the one-and two-fluid systems, Tellus 8 (1956), 460-471.

17. D. I. Meiron and P. G. Saffman, Overhanging interfacial gravity waves of large amplitude, J. Fluid Mech. 129 (1983), 213-218.

18. N. G. Meyers, An $L^{p}$-estimate for the gradient of solutions of second order elliptic divergence equations, Ann. Scuola Norm. Sup. Pisa Sér. (3) 17 (1963), 189-205.

19. R. M. Miura, The Korteweg-de Vries equation: a survey of results, SIAM Rev. 18 (1976), 412-459.

20. C. B. Morrey, Jr., On the analyticity of the solutions of analytic nonlinear elliptic systems of partial differential equations. I, Amer. J. Math. 80 (1958), 198-218; II 219-234.

21. _ Multiple integrals in the calculus of variations, Springer-Verlag, New York-Berlin, 1966.

22. L. Nirenberg, On elliptic partial differential equations, Ann. Sculoa Norm. Sup. Pisa Sér. (3) 13 (1959), 115-162.

23. A. S. Peters and J. J. Stoker, Solitary waves in liquids having non-constant density, Comm. Pure Appl. Math. 8 (1960), 115-164.

24. J. S. Russell, Report on waves, "Rep. 14th Meeting of the British Association for the Advancement of Science", p. 311, John Murray, London, 1844.

25. A. M. Ter-Krikorov, Théorie exacte des ondes longues stationnaires dans un liquide hétérogène, J. Mécanique 2 (1963), 351-376.

26. R. E. L. Turner, Internal waves in fluids with rapidly varying density, Ann. Scuola Norm. Sup. Pisa Sér. (4) 8 (1981), 513-573.

27. _ A variational approach to surface solitary waves, J. Differential Equations 55 (1984), 401-438.

28. R. E. L. Turner and J.-M. Vanden-Broeck, On the limiting configuration of interfacial gravity waves, Mathematics Research Center Technical Summary Report \#2800, University of Wisconsin-Madison, Madison, Wisconsin, Phys. Fluids 29 (1986), 372-375.

29. __ On solitary waves in two-fluid systems (to appear).

30. L. R. Walker, Interfacial solitary waves in a two-fluid medium, Phys. Fluids 16 (1973), 1796-1804.

31. G. T. Whyburn, Topological analysis, Princeton Univ. Press, Princeton, N.J., 1958.

Mathematics Research Center, University of Wisconsin-Madison, Madison, WISCONSIN 53706

Department of Mathematics, University of Chicago, Chicago, Illinois 60637 